LA W REN CE LIVERMORE N A TIO NAL LABORATORY

Forensic Analysis of samples from the Nuclear Fuel Cycle

L. Borg, I. Hutcheon

May 13, 2013 
This document was prepared as an account of work sponsored by an agency of the United States government. Neither the United States government nor Lawrence Livermore National Security, LLC, nor any of their employees makes any warranty, expressed or implied, or assumes any legal liability or responsibility for the accuracy, completeness, or usefulness of any information, apparatus, product, or process disclosed, or represents that its use would not infringe privately owned rights. Reference herein to any specific commercial product, process, or service by trade name, trademark, manufacturer, or otherwise does not necessarily constitute or imply its endorsement, recommendation, or favoring by the United States government or Lawrence Livermore National Security, LLC. The views and opinions of authors expressed herein do not necessarily state or reflect those of the United States government or Lawrence Livermore National Security, LLC, and shall not be used for advertising or product endorsement purposes.

This work performed under the auspices of the U.S. Department of Energy by Lawrence Livermore National Laboratory under Contract DE-AC52-07NA27344. 


\section{Forensic Analysis of samples from the Nuclear Fuel Cycle}

\section{Introduction}

This report summarizes the analytical techniques that can be used in forensic investigations of different materials produced across the nuclear fuel cycle in order to distinguish their origin and history. The measurable attributes of nuclear fuel cycle materials are known as signatures. Because signatures are created, modified and destroyed as material moves through the fuel cycle, it is important to consider the relevant analytical techniques in the context of where material is produced in the fuel cycle. To this end, we use the 10 steps in the fuel cycle identified by the IAEA as the basis for the following discussion.

Each step in the fuel cycle creates and/or modifies signatures. Table 1 summarizes the process, the material, the relevant signatures, and the analytical techniques commonly used to characterize the signatures for each fuel cycle step. From Table 1 it is apparent that most analytical techniques are relevant to evaluating signatures for a variety of materials along the fuel cycle. For example, isotopic compositions are important signatures for materials produced in every step in the fuel cycle. It is also clear from Table 1 that individual signatures can be evaluated using a variety of techniques. Uranium isotopes for example, can be determined using gamma-spectroscopy, quadrupole and multi-collector inductively coupled plasma mass spectrometry, thermal ionization mass spectrometry and $\mathrm{UF}_{6}$ gas source mass spectrometry. Each technique has its particular strengths and weaknesses. The appendix of this document provides a more detailed description of individual techniques that can successfully be applied to signature characterization for the range of fuel cycle materials.

\section{Important signatures}

Each step in the fuel cycle provides important signatures that can be used to constrain the source of materials. Below, short descriptions of these signatures are presented for each of the fuel cycle steps with an emphasis on the most important signatures for each process. This discussion is coupled to a discussion of the use of different analytical techniques to characterize/reveal the signatures

Geologic deposition. Uranium ores are the most common material associated with the first step in the fuel cycle. Important signatures associated with U-ores include mineralogy, bulk chemical composition and stable isotopic compositions. Mineralogy can be determined using x-ray diffraction or by optical microscopy by an experience microscopist. Elemental abundances in mineral phases can be estimated using secondary electron (SEM) and electron microprobe microscopy (EMP). This in turn can be used to further constrain the mineralogy. Textural

relationships between the minerals is best identified using an optical microscope or the SEM, whereas major, minor, and trace phases are best identified using the SEM. Quantitative chemical 
compositions of minerals are determined using the EMP. The bulk composition of the samples is determined using either $x$-ray fluorescence (XRF) or quadrupole/sector inductively coupled mass spectrometry (ICPMS) techniques. The XRF is useful for elements that are difficult to analyze by ICPMS such as $\mathrm{Si}, \mathrm{P}, \mathrm{Cl}, \mathrm{Br}$, and $\mathrm{S}$. It is also a relatively easy technique to obtain major element abundances, such as $\mathrm{Al}, \mathrm{Ti}, \mathrm{Mg}, \mathrm{Fe}, \mathrm{Ca}, \mathrm{Na}$ and $\mathrm{K}$. The ICPMS is able to determine a large number of trace elements, as well as most major element constituents in a geologic sample. The most important stable isotope is Sr. Strontium is best measured using thermal ionization mass spectrometer (TIMS), but can also be measure with the multi-collector ICPMS.

Mining and milling. The products of the mining and milling process are uranium ore concentrates (UOC). These include $\mathrm{U}_{3} \mathrm{O}_{8}$, ammonium diuranate, and sodium diuranate. These compounds are highly impure and have numerous potential signatures. Some of the most important signatures in UOC include the type of uranium compound that is present, the abundances of key trace elements in the UOC, the uranium and strontium isotopic composition of the UOC. The chemical form of the UOC is best determined using XRD, but can be constrained by the chemical composition of $U$ phases determined by SEM or EMP. The most important trace element signatures in UOC are the REE, which are best measured by ICPMS. Isotopic composition of $\mathrm{U}$ and $\mathrm{Sr}$, and in particular the ${ }^{234} \mathrm{U} /{ }^{238} \mathrm{U}$ and ${ }^{87} \mathrm{Sr} /{ }^{86} \mathrm{Sr}$ ratios, uniquely identify some UOCs. These isotopic ratios can be determined using either TIMS or multicollector ICPMS. The U isotopic compositions of the UOC can also be determined using alpha or gamma spectrometry.

Conversion. Conversion of UOC to $\mathrm{UF}_{6}$ involves further purification completed using either the wet or the dry process. The wet process removes impurities by solvent extraction, whereas the dry process does this by distillation in the final $\mathrm{UF}_{4}$ stage. Although the final product is very pure, intermediate materials, such as $\mathrm{UO}_{2}$ and $\mathrm{UF}_{4}$, do contain measurable quantities of trace elements impurities. Analysis of ultra- low abundances of trace elements can only be completed using ICPMS. The final product of the conversion process is $\mathrm{UF}_{6}$. This material is essentially devoid of impurities eliminating the potential for trace element signatures in this type of sample. As a consequence, isotopic signatures are the only signatures that remain. The uranium isotopic composition is expected to be essentially that of the initial $U$ ore mixed with whatever $U$ has been introduced into the processing stream as a result of contamination. Because conversion to $\mathrm{UF}_{6}$ removes $\mathrm{U}$ daughter products, the isotopic composition of the daughter isotopes, such as ${ }^{230} \mathrm{Th} /{ }^{232} \mathrm{Th}$, will reflect a combination of radiogenic ingrowth and contamination. The daughter isotopes are therefore likely to provide unique isotopic signatures of $\mathrm{UF}_{6}$. The $\mathrm{U}$ isotopic composition of the $\mathrm{UF}_{6}$ can be determined by $\mathrm{UF}_{6}$ mass spectrometry, TIMS, multi-collector ICPMS, or alpha and gamma spectrometry. The U daughter isotopes a likely to be present in small quantities and therefore are best analyzed by TIMS or MC-ICPMS,

Enrichment. Enrichment of $U$ can be accomplished using a variety of techniques, but gas centrifuge and gaseous diffusion are by far the most common. Additional processes that have been developed to various extents, but have never been commercialized, include AVALIS 
(Atomic Vapor Laser Isotope Separation), MLIS (Molecular Laser Isotope Separation), and the PSP (Plasma Separation Process). Enrichment of U by the SILEX process (Separation of Isotopes by Laser Excitation) is in the process of being developed for commercial enrichment. Uranium hexafluoride is the chemical form of the feed for the majority of these separation procedures (gas centrifuge, gaseous diffusion, MLIS, and SILEX). In all but the MLIS process the UF6 does not change chemical form. The primary signatures in $\mathrm{UF}_{6}$ are the naturally occurring uranium isotopes of ${ }^{238} \mathrm{U},{ }^{235} \mathrm{U}$, and ${ }^{234} \mathrm{U}$, as well as the non-natural isotopes of ${ }^{236} \mathrm{U}$, ${ }^{233} \mathrm{U}$, and ${ }^{232} \mathrm{U}$. The presence of non-natural $\mathrm{U}$ isotopes in the feed, waste, or products of the $\mathrm{U}$ enrichment stream indicates that at least some of the material came from previously irradiated $U$ fuel. Uranium isotopes can be measured using gamma spectrometry, TIMS or MC-ICPMS.

\section{U Fuel fabrication.}

\section{MOX fuel fabrication.}

\section{Receipt \& storage of fresh fuel at reactors.}

Irradiation. The most important signatures associated with irradiation are the isotopic compositions of spent fuel samples. Uranium and Pu isotopic compositions are used to evaluate the run conditions of the reactor. Because potential samples are run in different reactor types under different conditions, the isotopic composition provide signatures. Uranium and $\mathrm{Pu}$ isotopic compositions can be determined using either TIMS or multi-collector ICPMS. Note that trace abundances of some isotopes necessitate the use of mass spectrometers equipped with secondary electron multiplies. In addition to analysis of actinides, analysis of fission products, such as $\mathrm{Sr}, \mathrm{Zr}, \mathrm{Ba}$, and $\mathrm{Nd}$, are likely to provide unique signatures. This reflects the fact that the isotopic compositions of the fission products depend on reactor type and reactor performance. Strontium, $\mathrm{Ba}$, and $\mathrm{Nd}$ can be analyzed by either TIMS or multi-collector ICPMS, but $\mathrm{Zr}$ requires an MC-ICPMS. Internal normalization for mass fractionation occurring on the instrument cannot be applied to analysis of fission products because their isotopic compositions deviate dramatically from natural abundances. Thus, analysis of fission products requires characterization of instrument fractionation. Note that the isotopic composition of fission products is very different from natural isotopic compositions, and is likely to be highly variable. As a consequence, isotopic ratio measurements probably do not need to be made with the same level of precision as is typical for geologic samples.

\section{Reprocessing.}

\section{Nuclear waste reprocessing and storage.}


Table 1. Signatures in the Fuel Cycle

\begin{tabular}{|c|c|c|c|}
\hline Process & Materials & $\begin{array}{l}\text { Signatures } \\
\end{array}$ & Analytical Techniques \\
\hline $\begin{array}{l}\text { Geologic } \\
\text { Deposition }\end{array}$ & Ore & $\begin{array}{l}\text { Mineralogy, } \mathrm{U} \text { concentration, } \mathrm{U} \\
\text { isotopes, non-U isotopes, trace } \\
\text { elements }\end{array}$ & $\begin{array}{l}\text { OM, XRD, XRF, Q-ICPMS, } \\
\text { Photography }\end{array}$ \\
\hline $\begin{array}{l}\text { Mining and } \\
\text { milling }\end{array}$ & UOC & $\begin{array}{l}\text { Compound, U concentration, } U \\
\text { isotopes, non-U isotopes, trace } \\
\text { elements, morphology, size } \\
\text { distribution, color }\end{array}$ & $\begin{array}{l}\text { XRD, XRF, Q-ICPMS, MC- } \\
\text { ICPMS, TIMS, SEM, } \\
\text { Photography, Optical } \\
\text { Spectroscopy, IRMS }\end{array}$ \\
\hline Conversion & $\begin{array}{l}\mathrm{UF}_{4}, \mathrm{UF}_{6} \\
\mathrm{UO}_{2}, \mathrm{U}_{3} \mathrm{O}_{8}\end{array}$ & $\begin{array}{l}\text { Density, morphology, } \\
\text { compound, U content \& } \\
\text { isotopes, non-U isotopes, trace } \\
\text { element }\end{array}$ & $\begin{array}{l}\text { XRD, XRF, Q-ICPMS, MC- } \\
\text { ICPMS, TIMS, SEM, } \\
\text { Photography, Optical } \\
\text { Spectroscopy }\end{array}$ \\
\hline Enrichment & $\mathrm{UF}_{6}$ & $\begin{array}{l}\text { Density, morphology, } \\
\text { compound, U content \& } \\
\text { isotopes, trace elements }\end{array}$ & $\begin{array}{l}\text { Q-ICPMS, } \mathrm{UF}_{6}-\mathrm{MS}, \gamma- \\
\text { spectroscopy, } \alpha \text {-spectroscopy }\end{array}$ \\
\hline $\begin{array}{l}\text { U Fuel } \\
\text { fabrication }\end{array}$ & $\begin{array}{l}\mathrm{UO}_{2}, \mathrm{U}_{3} \mathrm{O}_{8}, \\
\text { metal, } \\
\text { pellets/rods, } \\
\text { waste }\end{array}$ & $\begin{array}{l}\text { Appearance, density, } \\
\text { roughness, morphology, } \\
\text { compound, U content \& } \\
\text { isotopes, trace elements }\end{array}$ & $\begin{array}{l}\text { TEM, SEM, OM, XRD, XRF, } \\
\text { Q-ICPMS, MC-ICPMS, TIMS, } \\
\text { IRMS, } \gamma \text {-spectroscopy, } \alpha- \\
\text { spectroscopy }\end{array}$ \\
\hline $\begin{array}{l}\text { MOX fuel } \\
\text { fabrication }\end{array}$ & $\begin{array}{l}\text { MOX } \\
\text { powder, } \\
\text { pellets, } \\
\text { rods, waste }\end{array}$ & $\begin{array}{l}\text { Appearance, density, } \\
\text { roughness, morphology, } \\
\text { compound, U/Pu content \& } \\
\text { isotopes, major \& trace } \\
\text { elements }\end{array}$ & $\begin{array}{l}\text { TEM, SEM, OM, XRD, XRF, } \\
\text { Q-ICPMS, MC-ICPMS, TIMS, } \\
\text { IRMS, } \gamma \text {-spectroscopy, } \alpha- \\
\text { spectroscopy }\end{array}$ \\
\hline $\begin{array}{l}\text { Receipt \& } \\
\text { storage of } \\
\text { fresh fuel at } \\
\text { reactors }\end{array}$ & $\begin{array}{l}\text { Fuel } \\
\text { assemblies }\end{array}$ & $\begin{array}{l}\text { Appearance, density, } \\
\text { compound, U/Pu content \& } \\
\text { isotopes, major \& trace } \\
\text { elements }\end{array}$ & $\gamma$-spectroscopy \\
\hline Irradiation & Spent Fuel & $\mathrm{U} / \mathrm{Pu}$ content $\&$ isotopes & $\begin{array}{l}\text { MC-ICPMS, TIMS }, \gamma- \\
\text { spectroscopy }\end{array}$ \\
\hline Reprocessing & $\begin{array}{l}\text { Pu-nitrate, } \\
\text { uranyl } \\
\text { nitrate, } \\
\mathrm{Pu}_{\mathrm{x}}, \mathrm{UO}_{\mathrm{x}} \\
\text { MOX }\end{array}$ & $\begin{array}{l}\text { Appearance, density, } \\
\text { compound, U/Pu content \& } \\
\text { isotopes, major \& trace element }\end{array}$ & $\begin{array}{l}\text { TEM, SEM, XRD, XRF, Q- } \\
\text { ICPMS, MC-ICPMS, TIMS, } \gamma \text { - } \\
\text { spectroscopy }\end{array}$ \\
\hline $\begin{array}{l}\text { Nuclear } \\
\text { waste } \\
\text { reprocessing } \\
\& \text { storage }\end{array}$ & $\begin{array}{l}\text { Nuclear } \\
\text { waste forms }\end{array}$ & $\begin{array}{l}\text { Serial number, container type, } \\
\text { activity, dimensions, mass, } \\
\text { density, color, compound, } \\
\text { major elements and isotopes }\end{array}$ & $\begin{array}{l}\gamma \text {-spectroscopy, XRD, Q- } \\
\text { ICPMS, MCICPMS, TIMS }\end{array}$ \\
\hline
\end{tabular}

Abbreviations as follows: $\mathrm{OM}=$ optical microscopy, $\mathrm{SEM}=$ scanning electron microscopy, TEM = transmission electron microscopy, XRD = x-ray diffraction, $\mathrm{XRF}=\mathrm{x}$-ray fluorescence, $\mathrm{Q}-\mathrm{ICPMS}=$ quadrupole inductively coupled mass spectrometry, MC-ICPMS = multi-collector inductively coupled mass spectrometry, TIMS = thermal ionization mass spectrometry, IRMS = isotope ratio mass spectrometry 
Discussion of Analytical Techniques

Photography

Optical Spectroscopy

Optical Microscopy

Scanning Electron Microscopy

Electron Probe Microscopy

Transmission Electron Microscopy

X-ray Diffraction

Secondary Ion Mass Spectrometry (SIMS)

Modified Davies-Gray Titration

Quadrupole Inductively Coupled Mass Spectrometry

X-ray Fluorescence

Thermal Ionization Mass Spectrometry

Multi-collector Inductively Coupled Mass Spectrometry

Isotope Ratio Mass Spectrometry

UF $_{6}$-Mass Spectrometry

High Resolution Gamma Spectrometry

Alpha Spectrometry

Stable Isotope Mass Spectrometry 


\section{Photography}

\section{Introduction}

The saying 'A picture is worth a thousand words' is very true and thus having photographic documentation of samples is very valuable in preserving an abundance of information some of which may not have originally appeared to be significant. Given how ubiquitous digital cameras are in todays' society, obtaining photos is rather convenient and any photo is better than none at all. However, this section will provide some guidance for taking photographs of samples that can help provide reliable and consistent information.

\section{Instrumentation}

\section{Camera}

The most obvious piece of equipment is the camera which an be a simple point-and-shoot type or cell phone or a high-end digital SLR camera with interchangeable lenses (see Figure 1). The cost of these can range from a few hundred dollars to several thousand dollars. Some of the key features to look for are the number of pixels and the lenses. More pixels will provide better spatial resolution and it's common to have more than 5 megapixels. Most cameras will allow for different photo features, however, too much alteration of the image can alter the data that can be collected. Adjusting the light level or exposure time, white balance, and aperture setting are valuable features.

\section{Tripod and Lighting}

A stable tripod is used to position the camera and keep it steady while lighting adjustments are being made. Using the delay timer, allows the user to take the hands off the camera during the photo and minimizes blurring due to vibrations.

The light source will have an effect on the perceived color of the material. Blackbody radiators such as incandescent light bulbs and tungsten halogen lamps provide continuous illumination across a broad wavelength spectrum and are preferred. Fluorescent or LED sources, although they may appear white, are not continuous and have different bands across the color spectrum and are not

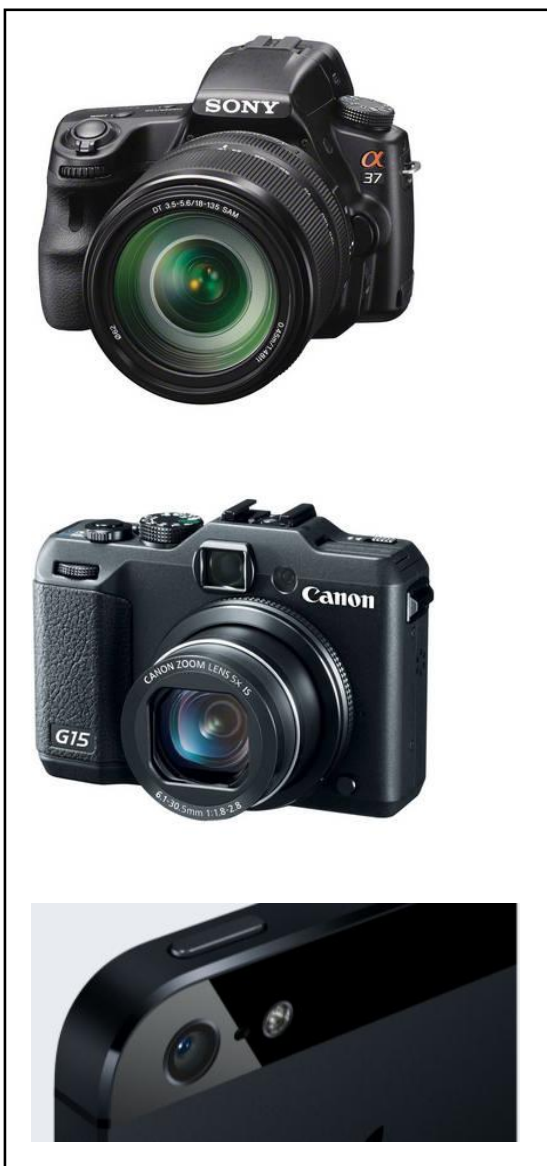

Figure 1. Camera options. ideal. These Lighting should be arranged to provide uniform illumination to the sample minimize shadows and avoiding hotspots. Diffusers can be used to help evenly distribute the light and sources should be at a consistent color temperature to cover a broad spectrum of light. 


\section{Reference/calibration}

Since all lighting conditions will vary, it is important to have a standard reference material to which all the images can be compared. One standard is known as the Macbeth Color Checker, Figure 2, which consists of 24 color squares arranged in a grid. The colors represent various natural objects, colors that are problematic for color reproduction, additive and subtractive primaries, and a gray scale. These charts come in a standard size, 8"x 11", or a mini version of 2.25 " $\mathrm{x} 3.25$ ". In addition to providing a color reference, the color chart can provide a sense of scale when the objects

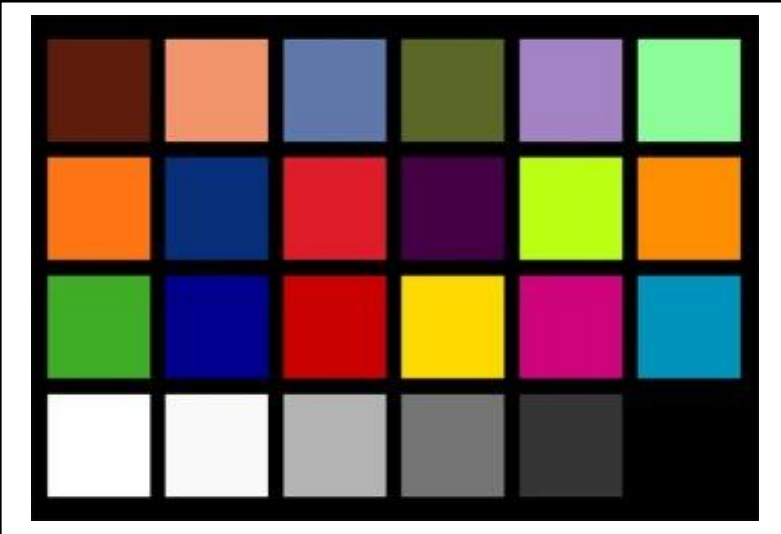

Figure 2. MacBeth Color Checker. are variable in size. Including a ruler in the image can provide a size reference that is more universally interpreted.

\section{Sample label}

All samples should have a reference number or identification label. It is best to use these numbers directly in the image and not rely on cross-referencing the image numbers to the samples. Figure 3 below shows a typical image of a uranium ore concentrate (UOC) sample in a

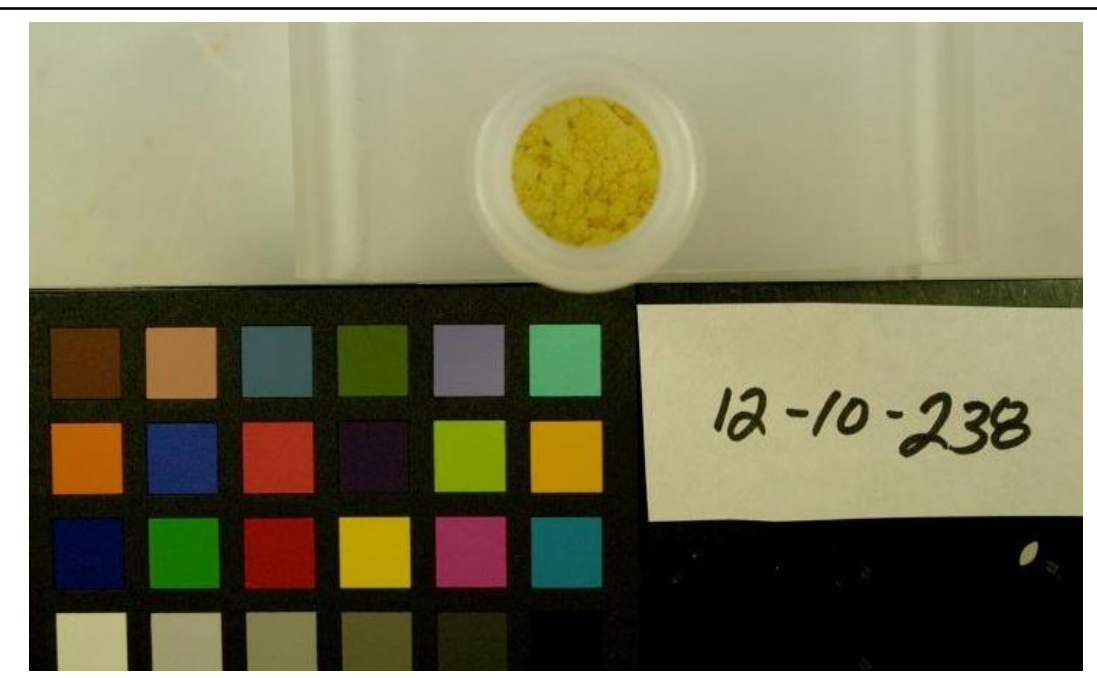

Figure 3. Typical image of a uranium ore concentrate standard $20 \mathrm{ml}$ vial with the color chart and sample ID label.

\section{Summary}

Photo documentation of samples can provide a valuable archive of information about the material prior to processing. Color, texture, size, and homogeneity can all be captured in a simple photograph for permanent record. 


\section{Optical Spectroscopy}

\section{Introduction}

Optical Spectroscopy (OS) is used to obtain molecular information on a bulk sample by measuring the light transmitted or reflected from the samples. The light is separated into discrete wavelengths and absorptions bands are measured at a detector. Different molecules or subgroups will have unique absorption bands based on their chemical composition. There are a number of different optical spectroscopy techniques that can be used, including; visible, near-infrared (NIR), infrared (IR), Raman, and fluorescence.

In this guide, we will focus on visible/NIR (Vis/NIR) and IR spectroscopy in a reflectance mode. The visible wavelength range is from $400-700 \mathrm{~nm}$ and the NIR covers 700$2500 \mathrm{~nm}$, while the IR is normally referred to in wavenumbers from $4000-500 \mathrm{~cm}^{-1}$. In general, the IR measures molecular motions associated with bending, wagging, twisting and stretching and have rather discrete bands associated with the different chemical entities (Figure 1).

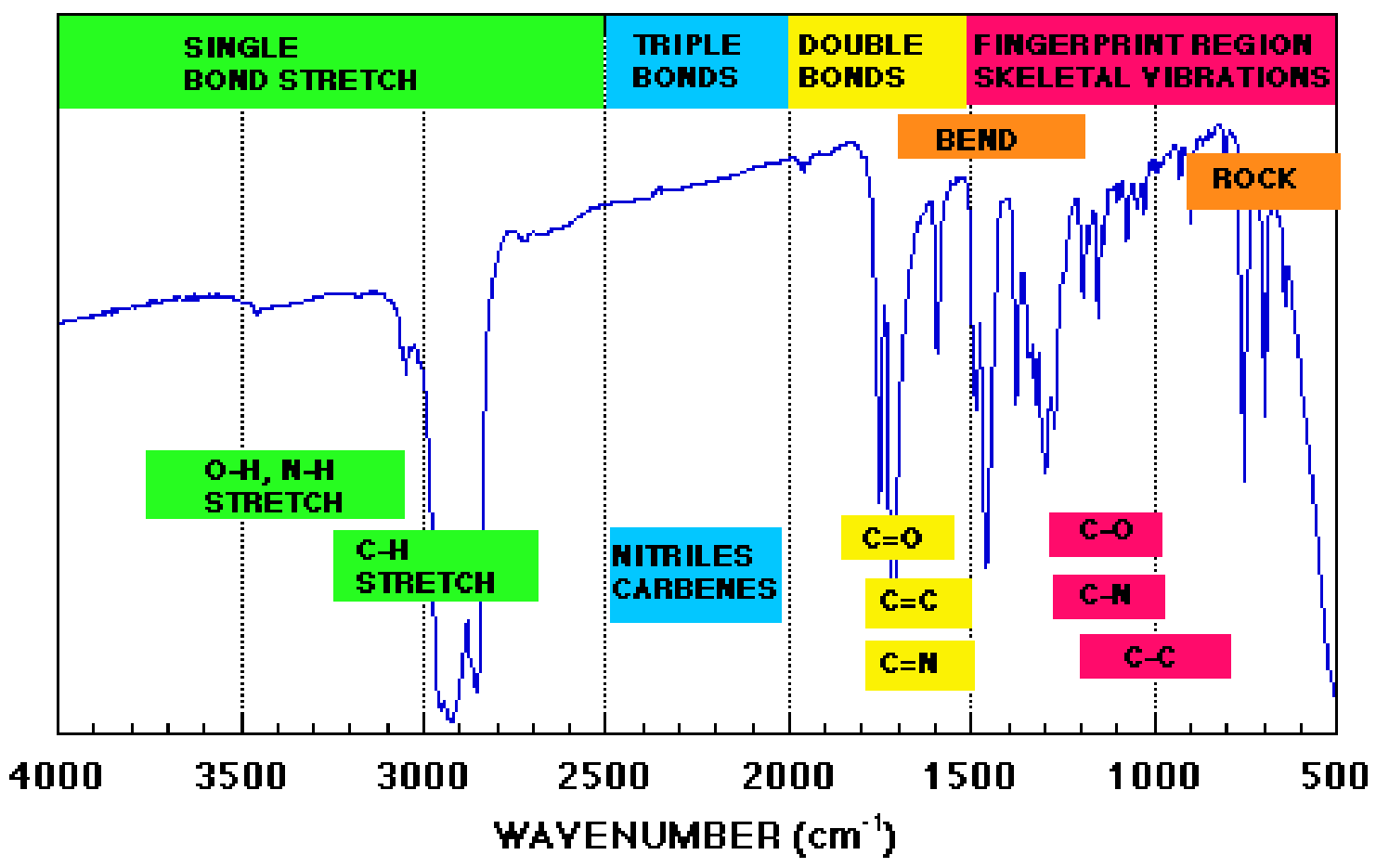

Figure 1. Illustrates some of the locations of spectral bands.

The NIR spectral range measures the overtones and combination bands of the motions detected in the IR. These absorption bands tend to be weaker and broader, yet can still provide 
important chemical information. Figure 2 is a chart that outlines the absorption band locations for chemical entities in the NIR spectral range.

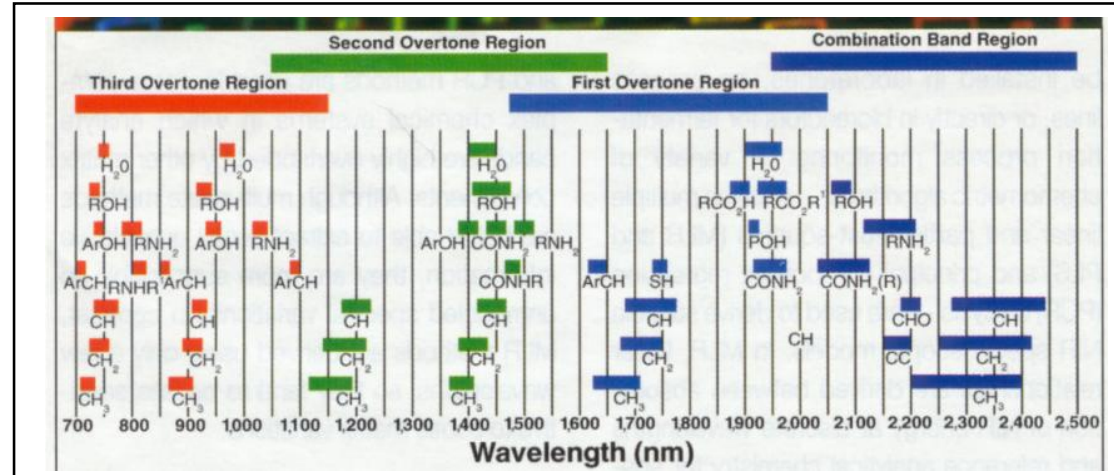

Figure 2 Location of absorption bands for selected chemical entities.
Both of these techniques offer advantages based on the information obtained and the method of analysis. . Vis/NIR spectrometer use standard quartz glass optics and can use fiber optics to measure the sample in place, whereas the IR instrument requires special materials

for the optics and the sample must be in direct contact with the ATR surface. These instruments are available from a number of manufacturers and can range from $\$ 40-60 \mathrm{~K}$.

\section{Instrumentation}

Vis/NIR spectrometers (Figure 3) can be purchased with or without the light source in a single instrument. The light is coupled into a bifurcated fiber optic bundle that delivers the light to sample and returns the reflected light to the spectrometer. Using fiber optics provides flexibility in making measurements and allows for samples to be analyzed in place if necessary. The measurements are non-contact and typically made at a few $\mathrm{mm}$ from the distal end of the

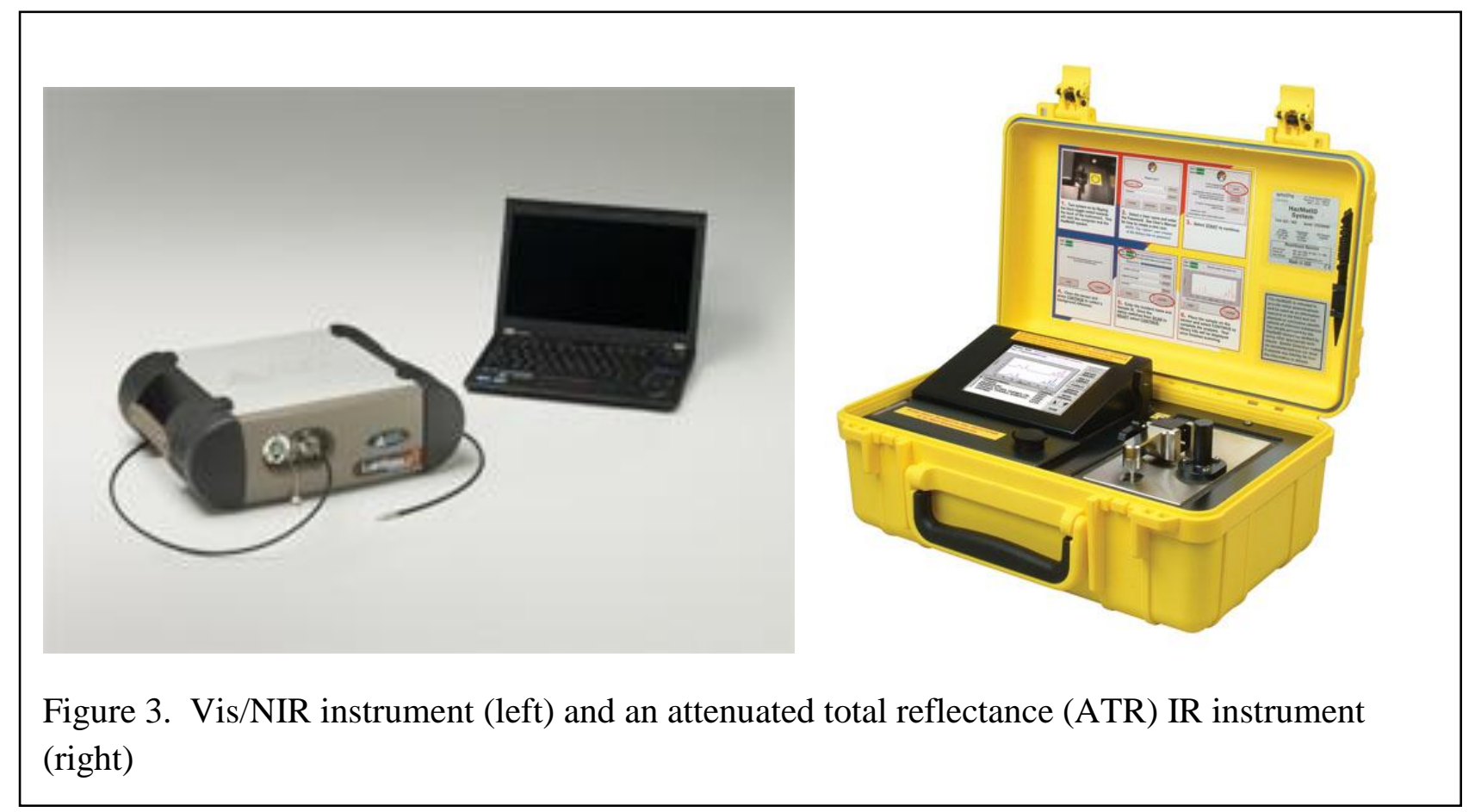


fibers. Smaller diameter fiber bundles can provide better spatial resolution, albeit still on the mm scale, and using optics measurements can be made at standoff distances as needed. For example, using a simple lens coupled to a fiber bundle, Vis/NIR reflectance spectra of UOC materials can be acquired directly in the sample vial as received without the need for sample preparation or taking a subsample.

IR spectrometers can be benchtop or portable models which are all in one instruments with the computer on board. Samples can be analyzed in reflectance or transmittance, however, the simplest method is to use attenuated total reflectance (ATR) to measure reflectance. ATR requires that the sample be in intimate contact with ATR surface which requires that the sample be pressed onto the surface with some force. This typically requires the sample be in a powder form and, although it is not a destructive analysis, the small amount of sample required is difficult to retrieve and potentially contaminated for subsequent analyses.

Light Source. The most common source used for the Vis/NIR range is a tungsten halogen bulb which has a continuous spectral output from 350- $2500 \mathrm{~nm}$. This is couple into low $-\mathrm{OH}$ fiber optics using quartz lenses. Sources in the IR are commonly glowbars which are essentially heated filaments that provide blackbody radiation output that follows Planck's law. Optics for IR transmission are commonly made from salt glasses and the ATR crystal is made from diamond or $\mathrm{ZnSe}$.

Light separators. Vis/NIR spectrometers use dispersive gratings to separate the light prior to the detector. The gratings can be fixed and the light imaged on to a diode array detector or moving which allows the light to be scanned over a single point fixed detector. There is an inherent advantage to having spectrometer with no moving parts which works well for the visible region where diode array detectors are readily available and sensitive. In the IR, interferometers provide substantial advantages (Felgett and Jacquinot advantages) over dispersive techniques in their ability to acquire all the light and all the wavelengths simultaneously.

Detectors. Silicone diode array detectors are readily available and sensitive from the visible up to $1000 \mathrm{~nm}$. From 1000-1800 nm InGaAs detectors are the most common, thinned InGaAs can extend the range to $2500 \mathrm{~nm}$. Although, there are InGaAs arrays available the single point source detectors with scanning spectrometer provide lower noise detection. Alternatively, $\mathrm{HgCdTe}$ detectors or arrays are available that can extend the spectral range to the IR, however, these can be considerable more expensive and require more energy due to the need for cooling. $\mathrm{HgCdTe}$ or dTGS point detectors are also used in the IR spectral range.

Sample preparation. One of the advantages of Vis/NIR spectroscopy is that it can be made on samples without any sample preparation. Thus, different veins in ore samples can be measured directly as a non- contact measurement. Grinding and homogenizing the sample helps to provide uniform response for the bulk sample. Rotating the sample during the analysis helps to minimize effects from specular reflection and inhomogeneities. For IR spectroscopy using ATR, the 


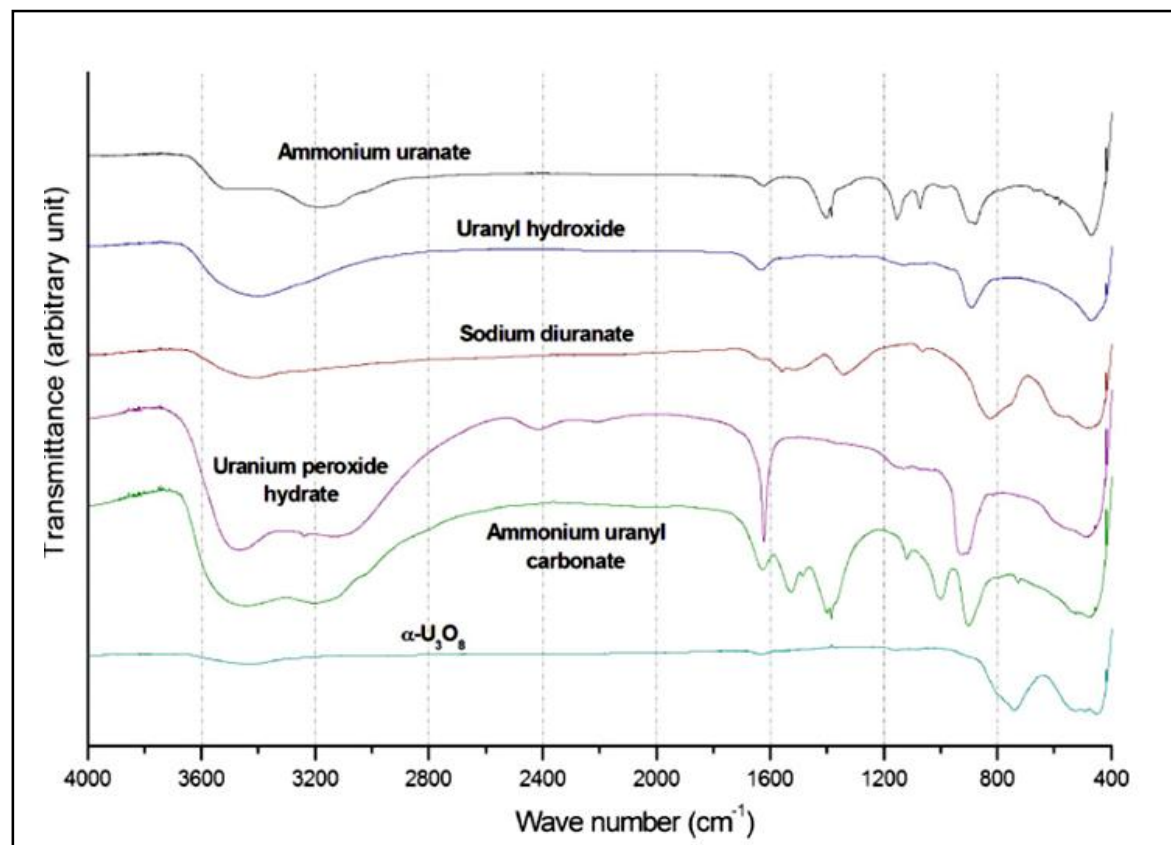

Figure 4. IR spectra of common uranium ore concentrates sample should be a powder as the light only penetrates on the order 3 microns and must be pressed smoothly onto the surface. Larger particles may not be analyzed appropriately if proper contact is not made with the ATR crystal.

\section{Analytical Issues}

IR spectroscopy measures fundamental molecular motions of the molecules and thus can

provide a means for chemical identification of pure compounds. In the case of processed UOC materials, there will be a mixture of chemicals which can complicate the IR spectra. Although the primary components can be identified (Fig. 4), comparison of different UOC sources will require the development of spectral libraries. Using chemometric algorithms such principal component analysis (PCA) or partial least squares (PLS), spectra can be rapidly classified as to how well they match those in the libraries. A visual comparison of the spectra is always recommended if conclusions are going to be made for source matching.

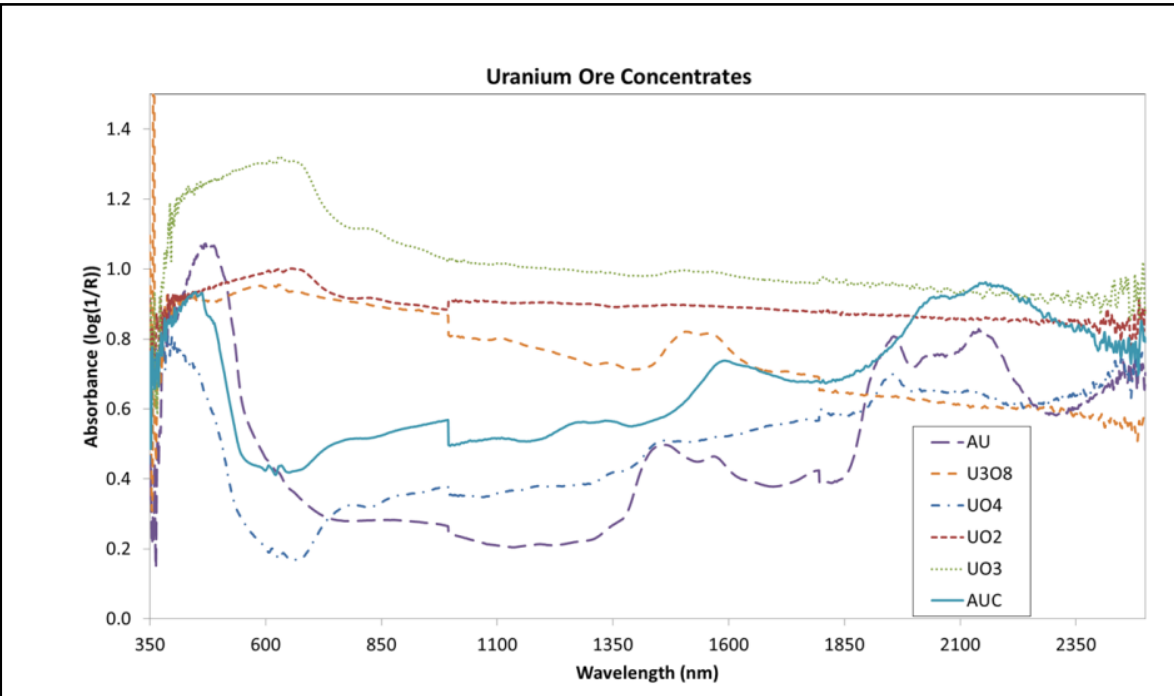

Figure 5. Unique spectra obtained in the Vis/NIR range
Vis/NIR spectroscopy can also provide chemical information about the samples, however, the combination and overtone bands are more challenging to interpret than the fundamentals. Thus, Vis/NIR spectroscopy is heavily reliant on techniques like PCA and PLS to compare spectra. Figure 5

shows some of the unique spectra obtained in the Vis/NIR range for a variety of UOC materials. 
NIR spectroscopy primarily measures $\mathrm{CH}, \mathrm{NH}$, and $\mathrm{OH}$ overtone vibrations and combinations, yet unique absorption bands have been observed or $\mathrm{U}_{3} \mathrm{O}_{8}$ and $\mathrm{UO}_{3}$ which can be attributed to crystal field effects.

\section{Strengths and weakness of optical spectroscopy}

Strengths. Both Vis/NIR and IR spectroscopy can be used to provide molecular information about the samples. Even for mixtures, spectra from unknown samples can be compared to spectral databases that can provide information as to the source or processing of the material. Vis/NIR spectroscopy is truly a non-contact, non-destructive method of analysis and can be used for remote detection. Spectra can be acquired rapidly and samples can be analyzed in place with fiber optics. IR spectroscopy can provide molecular information from the analsyis of the fundamental vibrations observed. Reflectance analysis using ATR is a convenient method that requires minimal sample preparation.

Weaknesses. Probably the biggest weakness of both Vis/NIR and IR spectroscopy is the limit of detection which is on the order of $1-10 \%$. There are additional methods that can be combined with these spectroscopic methods to improve the LODs. Analysis of mixtures requires that spectra of pure components in the mixture are in the spectral library. If the desired result is to compare mixtures, than this is not considered a weakness as composite spectra are compared. Finally, spatial resolution is limited to the area of the light source on the sample that is on the order of a 1-3 mm. 


\section{Optical Microscopy}

\section{Introduction}

Optical microscopy is an important technique for examination of bulk samples and particles. Optical microscopes are used to rapidly observe and compare morphology of bulk samples and particles, and also to locate and isolate impurities that may carry important forensic information. Optical microscopes are particularly useful for documentation of gross sample characteristics, assessment of sample heterogeneity, quantification of sample dimensions or features, and identification of areas of interest for further, higher resolution imaging.

\section{Instrumentation}

Two main types of optical microscope have applicability to nuclear forensic applications: stereoscopic microscopes and polarizing light microscopes. Most common are steroscopic (also called dissecting) microscopes that are specifically designed for low magnification observation of a sample using incident light illumination. These microscopes have two separate optical paths, and two eyepieces allowing slightly different viewing angles for each eye. This produces a thee-dimensional visualization of the sample being observed, and allows for examination of

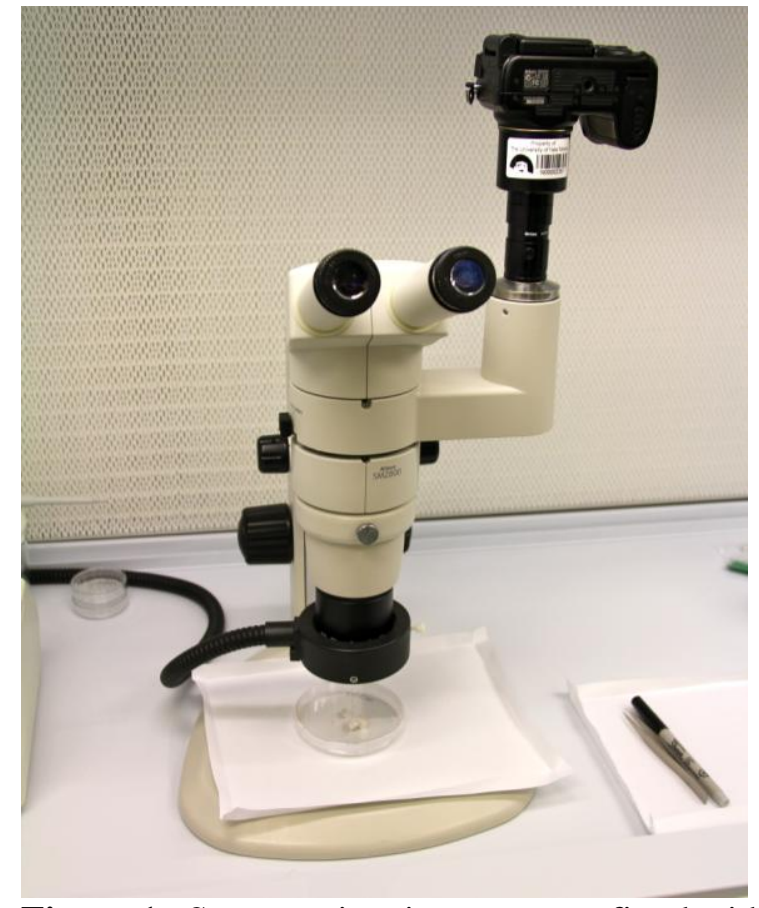

Figure 1. Steroscopic microscope outfitted with LED ring illuminator light source and confocal digital camera (microscope model is a Nikon SMZ800). This scope is set up in HEPA filtered clean bench. complex surface topography. A high quality, research grade steroscopic microscope costs about from $\$ 2500.00$ to 10,000 USD.

Visible light can be either incident to the sample, such as the illumination associated with a steroscopic microscope. Visible light can can also be transmitted through (or reflected from the surface of) a sufficiently thin and polished sample. Polarizing light microscopes use transmitted or reflected light to provide addiontal important forensic information. Minerals and other crystalline materials (organic as well as inorganic) produce interesting optical effects with crosspolarized light due to the variable birefringence of different materials. Polarizing light microscopes operate at higher magnification than stereoscopic microscopes and provide information on morphological, optical, chemical and physical properties, and are therefore especially useful for identifying rocks and minerals in thin section. Optical 
properties such as refractive index, birefringance, pleochroism, and interference figures can be used to identify crystals. Polarizing light microscopes use either transmitted or reflected polarized light, and can be used to study both translucent and opaque materials in thin section. These microscopes are designed to observe specimens that are visible primarily due to their optically anisotropic character. Polarizing light microscopes are equipped with a polarizing filter (polarizer) positioned in the light path between the light source and the specimen, and a second polarizer (called the analyzer) positioned in the optical pathway between the objective rear aperture and the observation tubes (oculars) (Figure 2). Image contrast arises from the interaction of plane-polarized light with birefringent (doubly refracting) specimens to produce two individual light wave components that are each polarized into mutually perpendicular planes. Because the velocities of these components are different, and vary with the propagation direction through the specimen, the components exit the specimen out of phase with each other. The waves are then recombined with constructive and destructive interference when they pass through the analyzer. A research quality polarizing light (petrographic) microscope costs about $\$ 5,000$ up to more than 20,000 USD. Cameras and imaging software are available for both steroscopic and polarizing light microscopes from all major vendors, and offer a wide range of tools for image quantification and analysis. These imaging systems generally cost from $\$ 5000$ and up, although very simple imaging systems can be as inexpensive as about $\$ 100$.

\section{Applications of steroscopic microscopy to forensic analysis.}

\section{Sample preparation.}

Samples for investigation by stereoscopic microscope require no special preparation. Bulk samples (i.e. yellowcake powders, U metal, rock samples, etc.) are typically placed in a suitable container (i.e. weighing boat) and visually inspected.

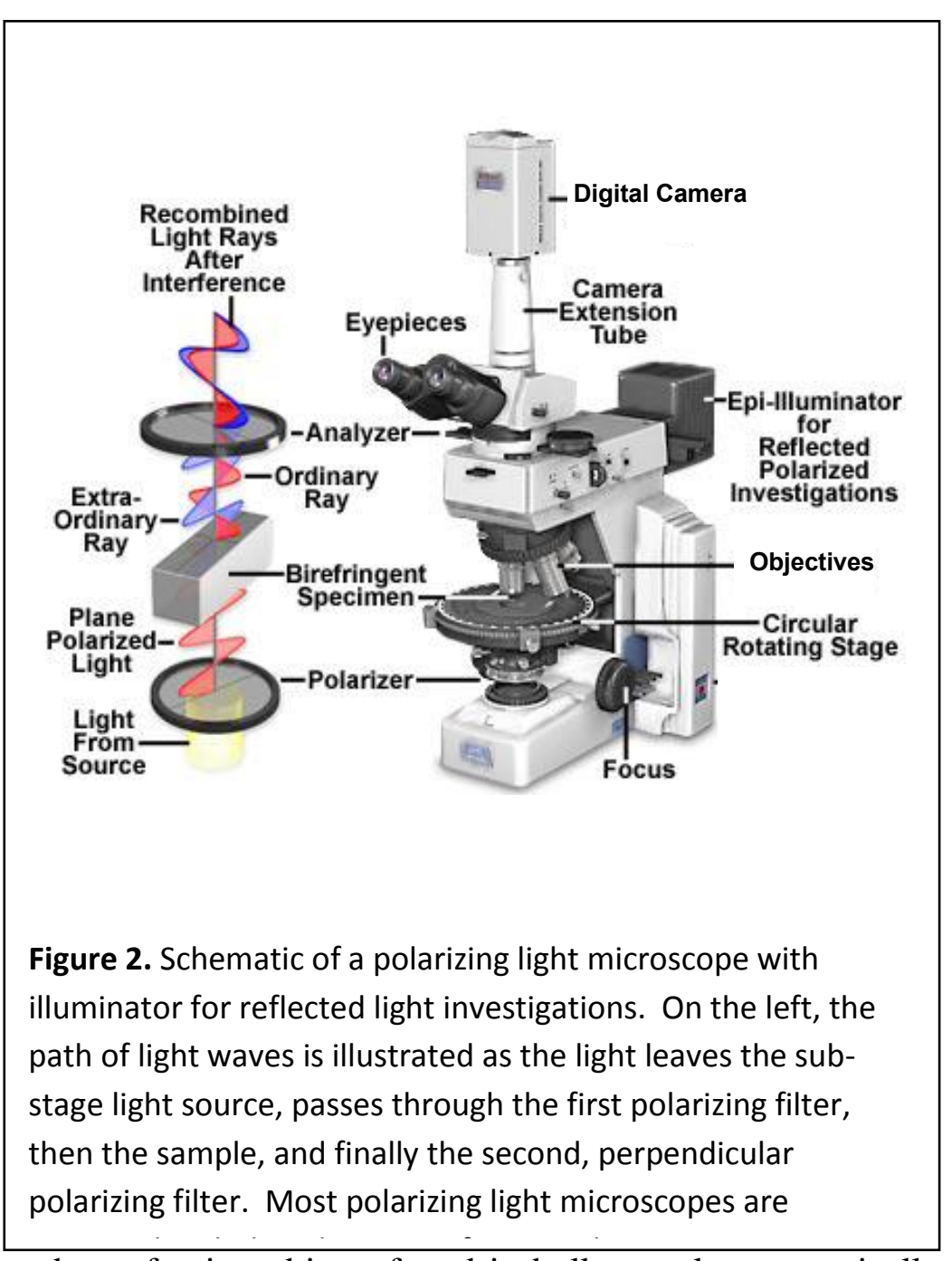
Textural characteristics are noted, and any foreign objects found in bulk samples are typically 
documented and removed. Samples for investigation by polarizing microscope must be mounted on glass slides, for analysis of samples in reflected light doubly polished thin sections are required. Thin sections for petrographic work are typically 30 um in thickness, although thicker sections that could also be used for other analytical techniques (i.e. SIMS, electron microprobe, laser ablation ICP-MS, micro-drilling) are sometimes preferred. Thin sections of samples can be prepared in-house if equipment is available, or can be sent out to commercial thin section preparation laboratories, although not all such laboratories are equipped to handle radioactive samples.

\section{Strengths and weakness of Optical Microscopy techniques}

Strengths. Stereoscopic observations of samples require essentially no sample preparation, and can yield important clues to material provenance including not only textures and appearance, but also the presence of foreign objects. Optical microscopy of bulk samples can be achieved rapidly, and can help to document and characterize forensic samples microscopically. Minimal training is required to make valuable stereoscopic observations, and it is straightforward to take high quality digital images of samples. Polarized light microscopy excels at aiding in the identification of mineral phases, and is particularly suited to the observation of opaque phases that might otherwise be difficult to identify. Reflected light microscopy can be useful for identifying regions of particular interest in a sample to be later interrogated by quantitative techniques including electron microprobe or SIMS.

Weakness. Optical microscopy, while inexpensive, rapid, and potentially quite useful in forensic investigations, has a couple of major weaknesses. First, Optical Microscopy is not quantitative, and therefore is mainly useful for documenting initial observations. Polarized light microscopy, and particularly reflected light microscopy used for identifying opaque phases, is less rapid than stereoscopic observations and requires expert sample preparation and also significant user training and experience. 


\section{Scanning electron microscopy}

\section{Introduction}

Scanning electron microscopy (SEM) is a versatile and commonly utilized form of microscopy that is well suited for the observation of topological and morphological sample features on a nanometer to micrometer scale. In nuclear security and nuclear forensics applications, SEM provides a means to characterize the morphology of particles and their associated microstructure.

Application of the SEM provides two types of images (Figure $\mathrm{X}$ ). Secondary electron images provide excellent depth of field to aid in the study of morphological features. Backscattered electron images provide contrast as a function of the mean atomic number of the constituent atoms in the sample. Similarly, characteristic $\mathrm{x}$-rays are emitted through the excitation of individual atoms and can be used for the semi-quantitative analysis of sample composition on the micrometer scale (see section on the Electron Microprobe). In particular, backscattered electron imaging can be a useful tool to identify impurities or foreign debris of potential forensic value in an otherwise homogenous matrix.

\section{Instrumentation}

The SEM works by rastering electrons, generated through thermal and/or electrostatic means and focused into a beam using electromagnetic lenses, across an area of the sample (Figure Y). As the electrons interact
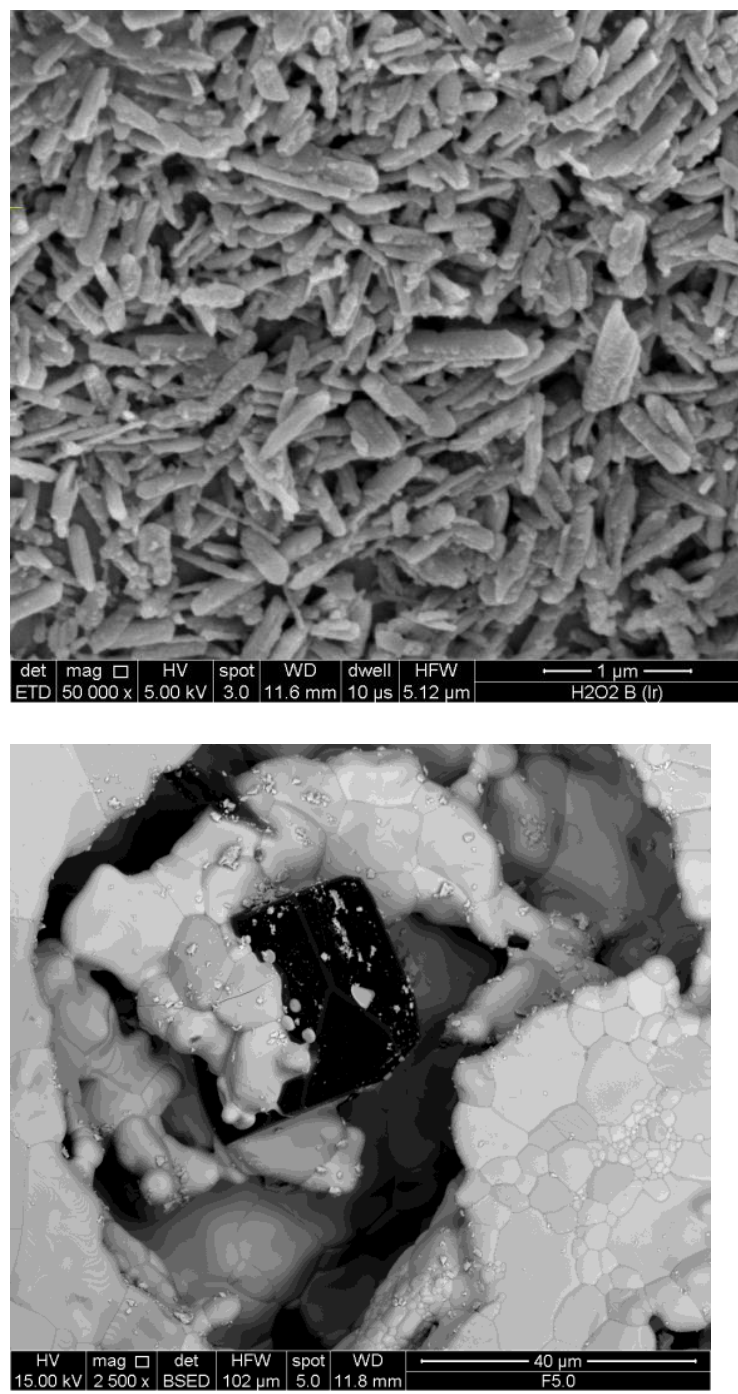

Figure X. Scanning electron micrographs demonstrating the utility of secondary electron imaging for morphology (top: uranyl peroxide) and backscattered electron imaging for identification of impurities (bottom: aluminum oxide impurity in an uranium dioxide ceramic). with the sample, useful signals are generated as secondary electrons, backscattered electrons, and characteristic X-rays, which are detected and transformed into an image (Figure Z).

\section{Application}

An analyst will typically use SEM images to perform a comparative examination of samples of interest against reference materials or other materials with an otherwise well-established pedigree. Effective comparisons necessitate sample preparation and imaging under similar conditions. Aspects to be compared include the sizes, shapes, and other morphological 


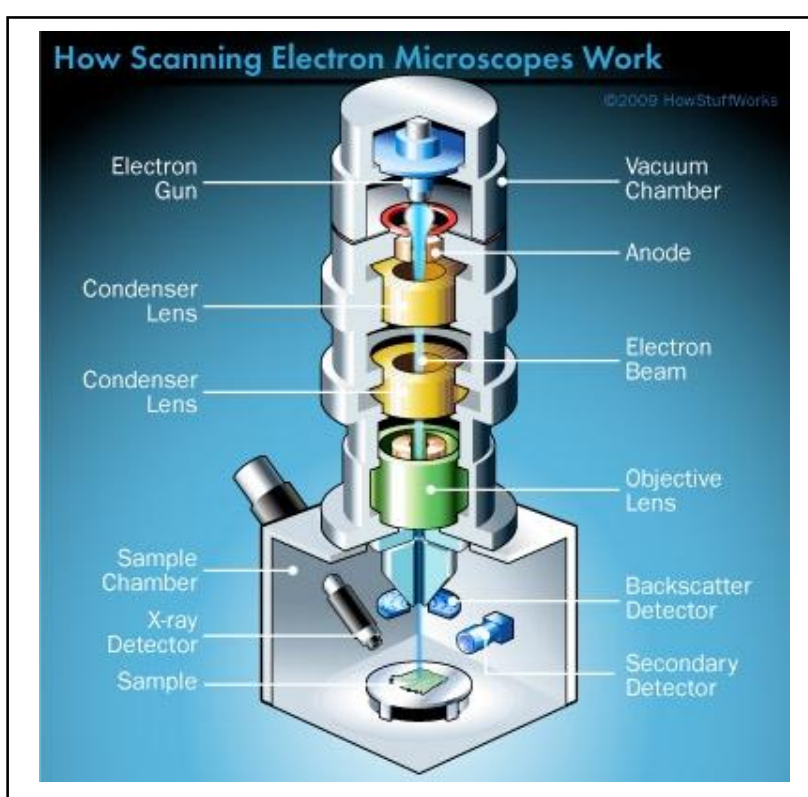

Figure Y. Schematic diagram of a Scanning Electron Microscope.

http://science.howstuffworks.com/scanning-electronmicroscope2.htm. [Accessed: 18-Nov-2012]. descriptors of the bulk materials and the associated microstructure. Morphology can indicate aspects of chemical and physical processing history.

Software is available to develop quantitative values for some morphological descriptors. Quantitative mage analysis is a complicated task and necessitates careful integration of sample preparation, imaging, and image processing methods. The analyst should thoroughly understand the limitations of the particular software and approach direct quantitative comparisons with caution.

\section{Sample Requirements}

For traditional SEM analysis, samples must be non-volatile to survive the vacuum environment and must be conductive to prevent charging by the electron beam. Nonconductive samples may be coated with a thin (typically a few tens of nanometers) layer of a

conductive substance such as carbon, gold, or iridium. These requirements can be eased using an Environmental SEM instrument, which can operate under various sample chamber atmospheres. Powdered samples consisting of a fraction of a milligram are typically adhered to a specimen stub using conductive tape, adhesives, or set into epoxy resin and polished. Analysts must take significant care to ensure that the sample is adequately dispersed. Common methods of dispersion include ultrasonication using various solvents and repeated contact transfers. Dispersion will depend heavily on the characteristics of the sample and require scoping efforts to ensure success.

\section{Strengths and Weaknesses}

Strengths. Alternatives to the SEM include optical microscopy, transmission electron microscopy, and optical light scattering techniques. The SEM offers superior resolution (typically at the nanometer range) and depth of field compared to optical microscopy, as well as simplified sample preparation compared to transmission electron microscopy. Optical light scattering techniques offer a potentially viable alternative; however, these systems tend to provide results that

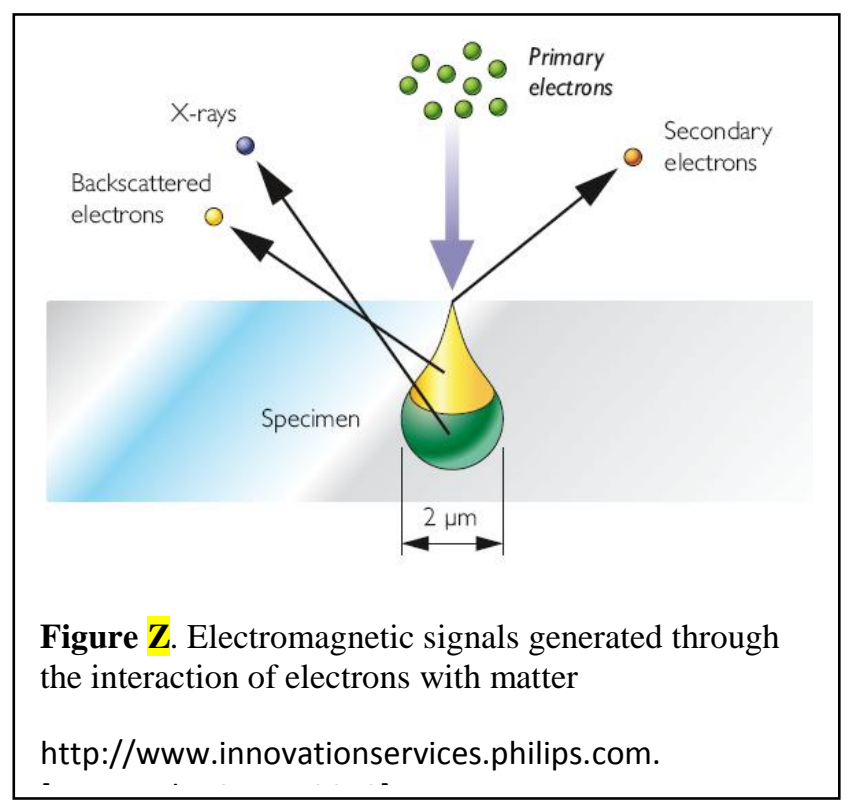


are abstractions of the actual particle dimensions. Sample size requirements of sub-milligram amounts for the SEM are also advantageous compared to most optical light scattering.

Weaknesses: The current state of maturity for the application of quantitative image analysis largely limits the use of SEM imagery to qualitative comparisons. Sample preparation, particularly dispersion, can be difficult for some materials. Further, capturing a representative set of images for a complex matrix can be difficult depending on the degree of sample homogeneity. 


\section{Electron Probe Microscopy}

\section{Introduction}

An electron microprobe (also called an electron probe microanalyzer or EPMA) is an electron microscope designed for the non-destructive x-ray microanalysis and imaging of solid materials. The technique is usually applied to inorganic materials such as metals, oxides, minerals, glasses, semiconductors, and ceramics. Organic materials may be analyzed but must be made stable in vacuum, freeze-dried, fixated with $\mathrm{OsO}_{4}$ or similar fixative, and treated as beam-sensitive materials. It is capable of high spatial resolution and relatively high analytical sensitivity. The electron microprobe can typically acquire digital secondary-electron and backscattered-electron images, digital x-ray maps, and sometimes digital cathodoluminescence images. Most of the periodic table can in principle be analyzed (Boron through Uranium), subject to several important considerations. The analytical sensitivity ranges from a low of a few parts per million for optimum cases, to a typical detection limit of several hundred ppm, but can be as high as several weight percent for problem elements. The volume sampled is typically a few cubic microns, corresponding to a weight of a few picograms. Samples must be prepared as flat, polished solid mounts (either rounds or petrographic thin sections), and must be clean and stable in a $10^{-5}$ torr vacuum environment. After preparation, samples are coated with a $\sim 22 \mathrm{~nm}$ layer of carbon using a high-vacuum carbon evaporator. The quality of analyses performed depends on the quality of sample preparation, character of the sample material, and availability of appropriate primary and secondary calibration standards for the desired elements.

\section{Instrumentation}

The electron microprobe works similarly to a scanning electron microscope (SEM). A solid sample is bombarded a focused beam of high-energy electrons $(5-30 \mathrm{keV})$ which causes a variety of different type of interactions between the electron beam and the atoms in the sample. A number of these interactions produce signals that can be detected and used to provide information about the nature of the material being examined. For forensic applications, the most important of these interactions occurs when the electron beam collides with an atom in the sample and causes an inner shell electron to be ejected from the atom. The inner shell vacancy leaves the atom in a high-energy excited state, and it loses this excess energy by either having an outer shell electron move to fill the inner shell vacancy (accompanied by the release of an X-ray photon) or the ejection of an outer shell (auger) electron. An X-ray produced by the interaction with an inner shell electron has an energy equal to the difference in energy between the two shells involved in the transition. For a given transition between a specific inner and outer shell, the energy of the X-ray will uniformly increase with increasing atomic number $(Z)$ of the excited atom. As a result, for a given transition, each element will produce $X$-rays with unique (characteristic) wavelength (energy). The electron microprobe uses these characteristic X-rays, ranging in energy from $0.1-15 \mathrm{keV}$ to identify and quantify the elements present in the sample. 


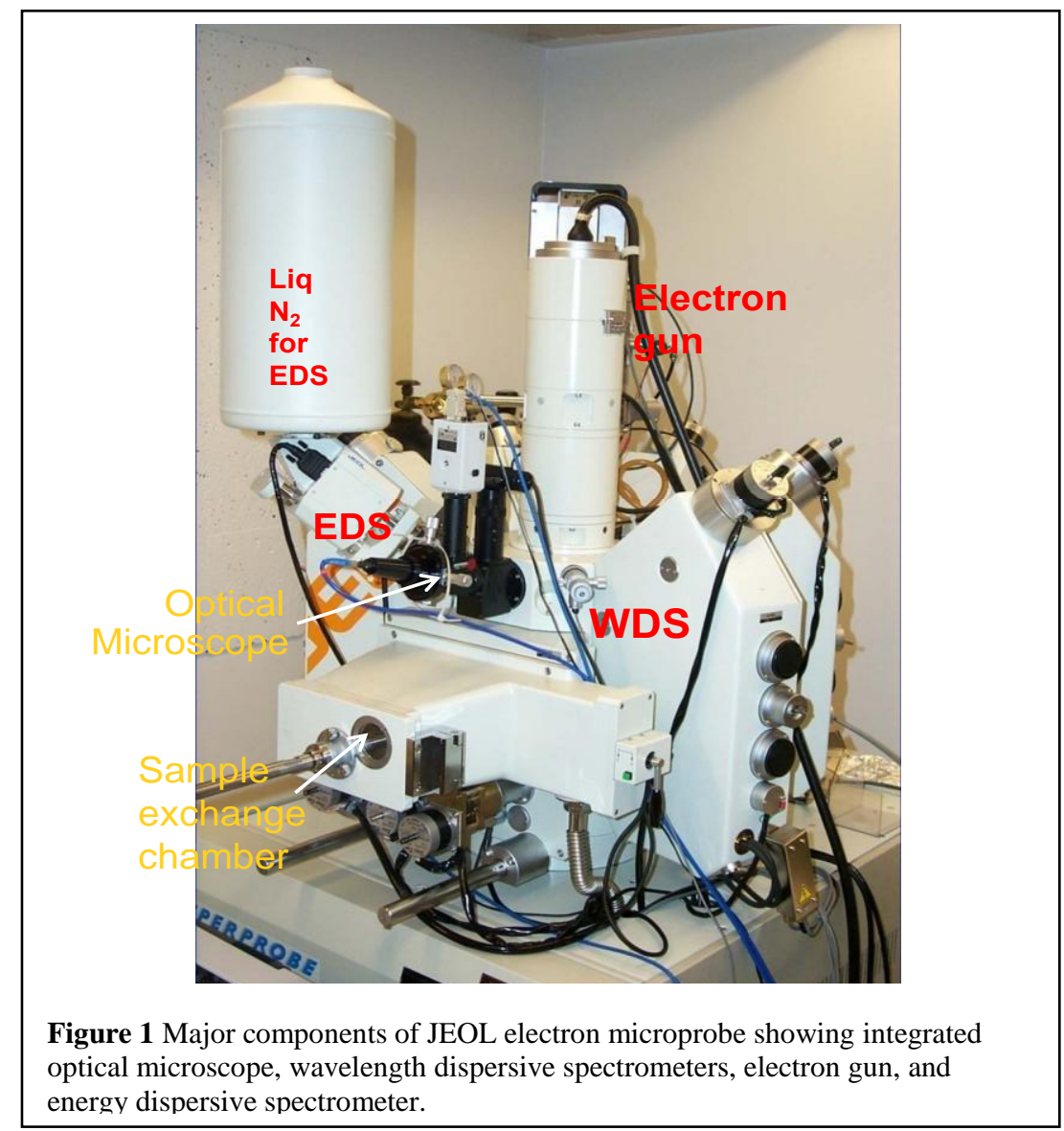

There are two methods for detecting and quantifying the spectrum of secondary X-rays emitted from the specimen: Wavelength dispersive spectrometry and Energy Dispersive Spectrometry. The wavelength dispersive spectrometry is a quantitative technique that employs a diffracting crystal to isolate the characteristic X-ray peaks. This technique is characterized by high spectral resolution (i.e. elements are clearly resolved) and better detection limits, although it is considerably slower than energy dispersive spectrometry. In contrast, energy dispersive spectrometry uses a solid-state detector that discriminates between the energies of incoming photons. This technique is characterized by low spectral resolution and lower precision, but it is valuable because it allows the simultaneous collection of the entire elemental spectrum, and is therefore allows rapid semi-quantitative characterization of sample composition.

These instruments have similar designs and roughly equivalent capabilities. Electron microprobes typically have four main components: (1) an electron column (gun) in which the electron beam is produced, (2) wavelength dispersive spectrometers (WDS) for detection and counting of characteristic X-rays, (3) an energy dispersive spectrometer (EDS) for rapid detection of the whole spectrum of measurable elements, and (4) counting electronics for amplifying the signal, screening out noise, and measuring the $\mathrm{X}$-ray count rate. Electron microprobes used for forensic analysis are obtained from commercial vendors at a cost of $\sim$ 0.75-1.5M US dollars (Figure 1). A schematic illustration of the beam paths in an electron microprobe is presented in Figure 2.

\section{X-ray lines and Quantitative Analysis.}

The electron shells of an atom are labeled $\mathrm{K}, \mathrm{L}, \mathrm{M}, \mathrm{N}$, and $\mathrm{O}$ starting with the innermost, lowest energy shell. These shells correspond to orbitals with principle quantum numbers $n=1,2,3$ etc. The transitions giving rise to the $\mathrm{X}$-ray lines most commonly used in electron microprobe 
analysis are the transitions Lii-K (the $\mathrm{K} \square$ line), Mv-Lii (the $\mathrm{L} \square$ line) and somewhat less commonly Nvii - Mv (the M $\square$ line) (Figure 3).

Characteristic X-rays are detected and counted by several wavelength dispersive spectrometers (WDS) arrayed around the sample chamber. Modern instruments usually have between 4 and 6 WDS installed. Each spectrometer contains a crystal that can be positioned at an angle to diffract a specific X-ray wavelength coming from the sample into the X-ray counter while allowing the other wavelengths to be absorbed by the crystal. The rate at which X-ray photons of a given element are produced by the sample can be determined by setting the crystal to the position which will reflect the X-ray of interest into the detector and then counting the number of X-ray photons detected over a specified length of time. The crystal of a WDS spectrometer can

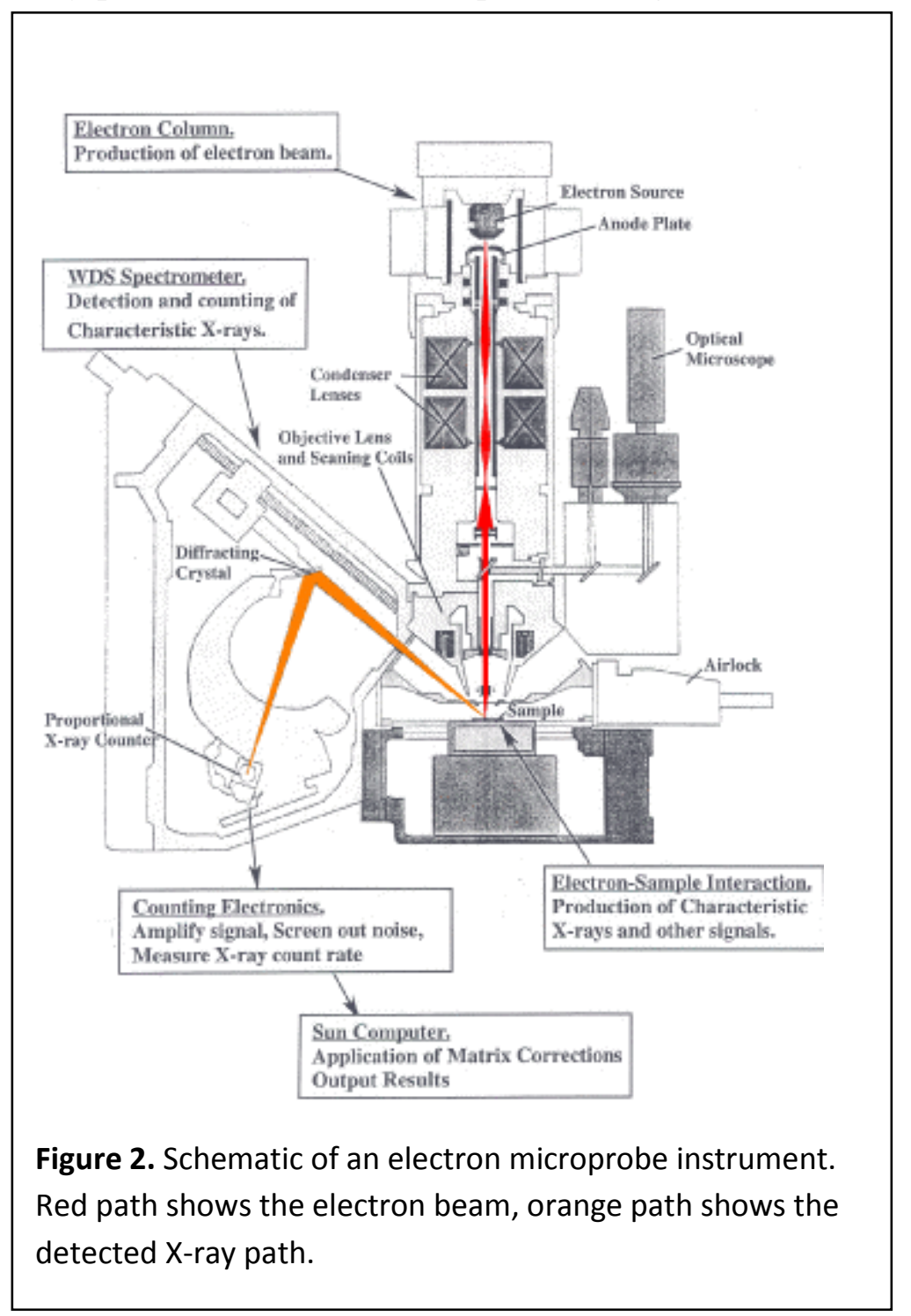
only collect X-ray counts for one wavelength at a time, and therefore the analysis of multiple elements on a single spectrometer must be done sequentially. The angle at which the spectrometer crystal is placed is dependent on Bragg's Law, as the diffraction angle changes with the wavelength being diffracted. Since no single crystal can satisfy Bragg's Law for the full range of $\mathrm{X}$-ray wavelengths for the entire periodic table, most modern electron microprobes are equipped with more than one spectrometer and the ability to automatically exchange different crystals within a single spectrometer. A typical electron microprobe might have four WDS spectrometers and as many as 12 diffracting crystals allowing the collection of X-ray counts for four elements simultaneously, and giving a range for all elements between $B$ and $U(Z=5-92)$.

Quantitative electron probe microanalysis is a standards-based technique. In order to turn the Xray count rate generated from an unknown into a concentration, it is necessary to compare the 
unknown count rate to the count rate obtained from a standard with a known composition, under the same analytical conditions. For example, if under identical analytical conditions an unknown sample produces 20 counts per second and the standard (containing $50 \% \mathrm{Fe}$ ) produces 40 counts per second, to a first approximation the unknown sample is calculated to have: $(20 / 40) * 50 \mathrm{wt} \%$ $\mathrm{Fe}=25 \mathrm{wt} \% \mathrm{Fe}$. In fact, this approximation must be adjusted to account for the effects of other
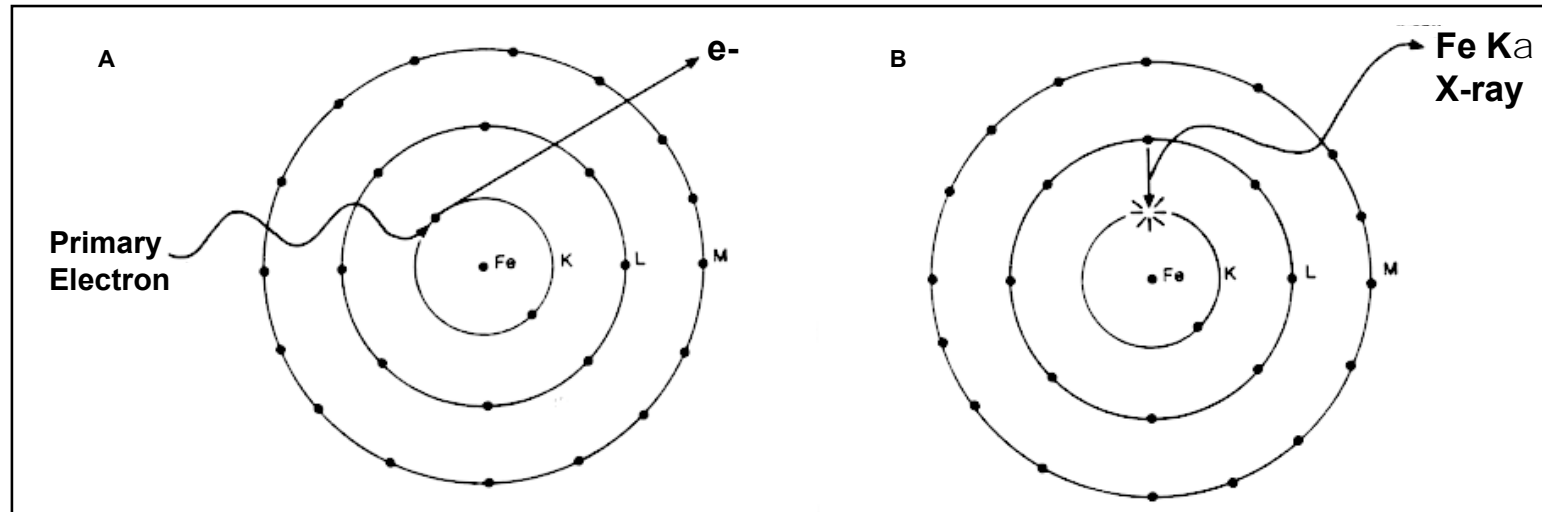

Figure 3. Excitation of a characteristic X-ray results from an atom of iron. A) the primary electron collides with the atom and causes an inner shell electron to be ejected, in this case an electron from the $\mathrm{K}$ shell. Since this leaves the atom in a high-energy state, an electron from the L shell replaces the inner shell electron, (B) and causes the emission of a Fe K $\square$ characteristic X-ray that can then be counted.

elements present in the sample and standard that may differently affect x-ray production for the element of interest (matrix effects). These corrections will be discussed below.

Semi-quantitative Energy Dispersive Analysis. Qualitative analysis of EDS spectra involves the identification of the lines in the spectrum, a task which is usually aided by software designed for identifying X-ray peaks. The technique allows for rapid identification of specimen composition, and is useful for understanding what materials are present prior spending the time for more time consuming WDS measurements. Unlike WDS, EDS is insensitive to specimen geometry, which means that a semi-quantitative analysis of an imperfectly prepared sample is possible. Energy dispersive spectrometers employ pulse height analysis: a detector giving output pulses proportional in height to the $\mathrm{X}$ ray photon energy is used in conjunction with a pulse height analyzer. A solid-state detector is

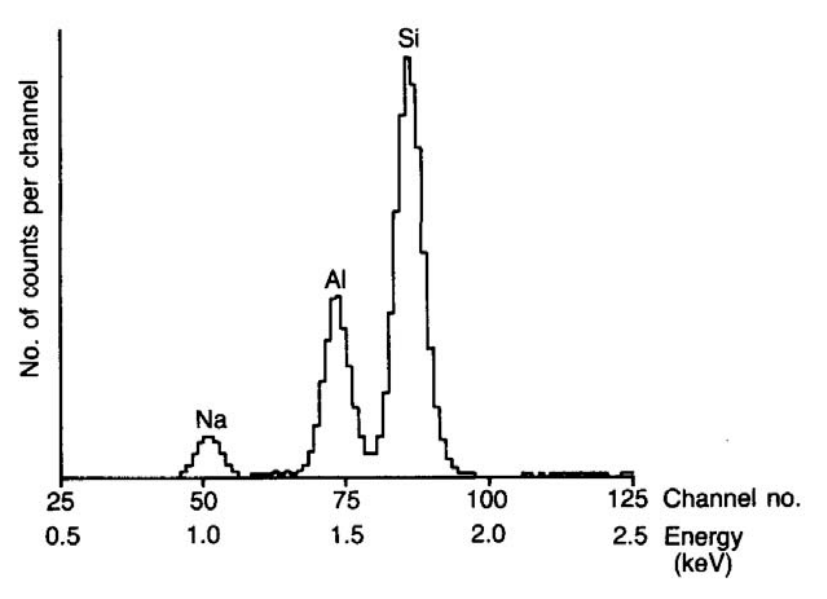

Figure 4 Energy Dispersive Spectrum of jadeite showing $\mathrm{K}$ peaks of $\mathrm{Na}, \mathrm{Al}$, and $\mathrm{Si}$. 
used because of its better energy resolution. Incident X-ray photons cause ionization in the detector, producing an electrical charge, which is amplified by a sensitive preamplifier located close to the detector. Both the detector and the preamplifier are cooled with liquid nitrogen to minimize electronic noise (Figure 2).

The EDS spectrum is displayed in digitized form with the $\mathrm{X}$-axis representing X-ray energy and the $\mathrm{y}$-axis representing the number of counts per channel (Figure 4). Although X-ray lines consist of effectively mono-energetic photons, they are broadened into Gaussian peaks due to the electronic response of the system. Energy resolution is defined as the full width of the peak at half maximum height. The resolution of an EDS is about an order of magnitude worse than WDS, but it is good enough to separate the $\mathrm{K}$ lines of neighboring elements.

\section{Analytical Issues}

The net intensity of the characteristic X-ray peak is proportional to the mass concentration of the element in the specimen. Quantitative analysis relies on comparing the specimen with a standard of known concentration of the element. Standards are calibrated to establish the net counts per unit concentration for each element. The standards are natural or synthetic materials with accurately known composition. These are usually pure stoichiometric compounds, or alternatively they have been meticulously analyzed by other techniques

Correction procedures. The ratio of peak intensities measured on the specimen and standard gives an approximate concentration $\mathrm{C}_{\text {specimin }}=\mathrm{C}_{\text {standard }} *\left(\mathrm{I}_{\text {specimin }} / \mathrm{I}_{\text {standard }}\right)$. A number of corrections must be applied because X-ray peak intensities are affected by differences in the nature and composition of the bulk material in the specimen and standard. The most widely applied correction procedure is called the ZAF correction. The letters stand for the following:

Z-atomic number (affects the penetration of incident electrons into the material)

A-absorption of X-rays in the specimen, on the path to the detector

F-fluorescence caused by other X-rays generated in the specimen

Since the corrections are a function of sample composition, the correction procedure necessarily involves iteration, starting with estimated correction factors and continuing until the input composition and the corrected composition converge. Corrections are usually handled by the microprobe software package.

Analytical uncertainty. Characteristic X-rays are emitted as a result of random events, so the standard deviation $(\square)$ of a set of counts can be approximated by the square root of the total number of counts.

For a sum or difference, $\sigma=\sqrt{\left(\sigma_{1}\right)^{2}+\left(\sigma_{2}\right)^{2}}$ 
For a ratio or product, where $m$ is the mean value: $\sigma / m=\sqrt{\left(\sigma_{1} / m_{1}\right)^{2}+\left(\sigma_{2} / m_{2}\right)^{2}}$

If the total counts on the peak and background are $\mathrm{N}_{\mathrm{p}}$ and $\mathrm{N}_{\mathrm{b}}$ respectively, (peak and background being counted for the same length of time), then: $\sigma_{\text {net peak }}=\sqrt{N_{p}+N_{b}}$

And the overall uncertainty on the concentration determined from the specimen-standard ratio will be: $\sigma_{\text {overall }}=m_{s p} \sqrt{\frac{\left(N_{p}+N_{b}\right)_{s p}}{\left(N_{p}-N_{b}\right)_{s p}{ }^{2}}+\frac{\left(N_{p}+N_{b}\right)_{s t d}}{\left(N_{p}-N_{b}\right)_{s t d}{ }^{2}}}$

Since many elements are present in moderate amounts compared to the high concentrations of the standards, the standard term will be comparatively small, so the analytical uncertainty can be approximated by:

$$
\sigma_{\text {overall }}=m_{s p} \sqrt{\frac{\left(N_{p}+N_{b}\right)_{s p}}{\left(N_{p}-N_{b}\right)_{s p}}}
$$

Typical values for the relative error $\square / \mathrm{m}$ are 0.3 to $1 \%$ on this basis for oxide concentrations between 50 and $2 \mathrm{wt} \%$. In practice, however, the reproducibility will not be as good as the relative error (e.g. Waters and Charnley, 2002).

Detection Limits. The detection limit is typically a concentration equivalent to three standard deviations of the background counts, corresponding to a $99 \%$ probability that a peak is significantly different from the background. If the count rate and counting time on the background are $\mathrm{I}_{\mathrm{b}}$ and $t_{\mathrm{b}}$ then:

$$
\sigma_{b}=\sqrt{\left(I_{b} * t_{b}\right)}
$$

To convert this to concentration, take the count rate per weight percent on the standard $\left(\mathrm{I}_{\text {std }}\right)$ and the ZAF correction factor $(\sim 1)$. The detection limit is then:

$$
D L(w t \%)=3 *(Z A F) * \frac{1}{I_{s t d}} * \sqrt{\left(\frac{I_{b}}{t_{b}}\right)}
$$

Detection limits for routine analysis of common elements (Na to $\mathrm{Fe}$ ) are around $0.02 \mathrm{wt} \%$.

\section{Other Useful Microprobe Capabilities}

Backscattered Electrons. Aside from characteristic X-rays, electron beam-sample interactions produce a number of other signals that can be detected by the electron microprobe. 
One of the most useful of these interactions is the production of backscattered electrons (BSE). A backscattered electron is a beam electron whose trajectory has been deflected so much by elastic scattering events with atoms in the sample that it is turned around and reemerges from the sample surface with relatively high energy. The number of beam electrons that are backscattered is strongly dependent on the average atomic number $(Z)$ of the atoms in the sample. Backscattered electron detectors are placed in the roof of the sample chamber, and rastering the electron beam over the sample surface makes it possible to produce a composition map showing areas of the sample having different average atomic numbers as different shades of gray (Figure 5). Backscattered electron images of this type are extremely useful for analyzing multiphase samples and for identifying impurities in otherwise homogeneous materials.

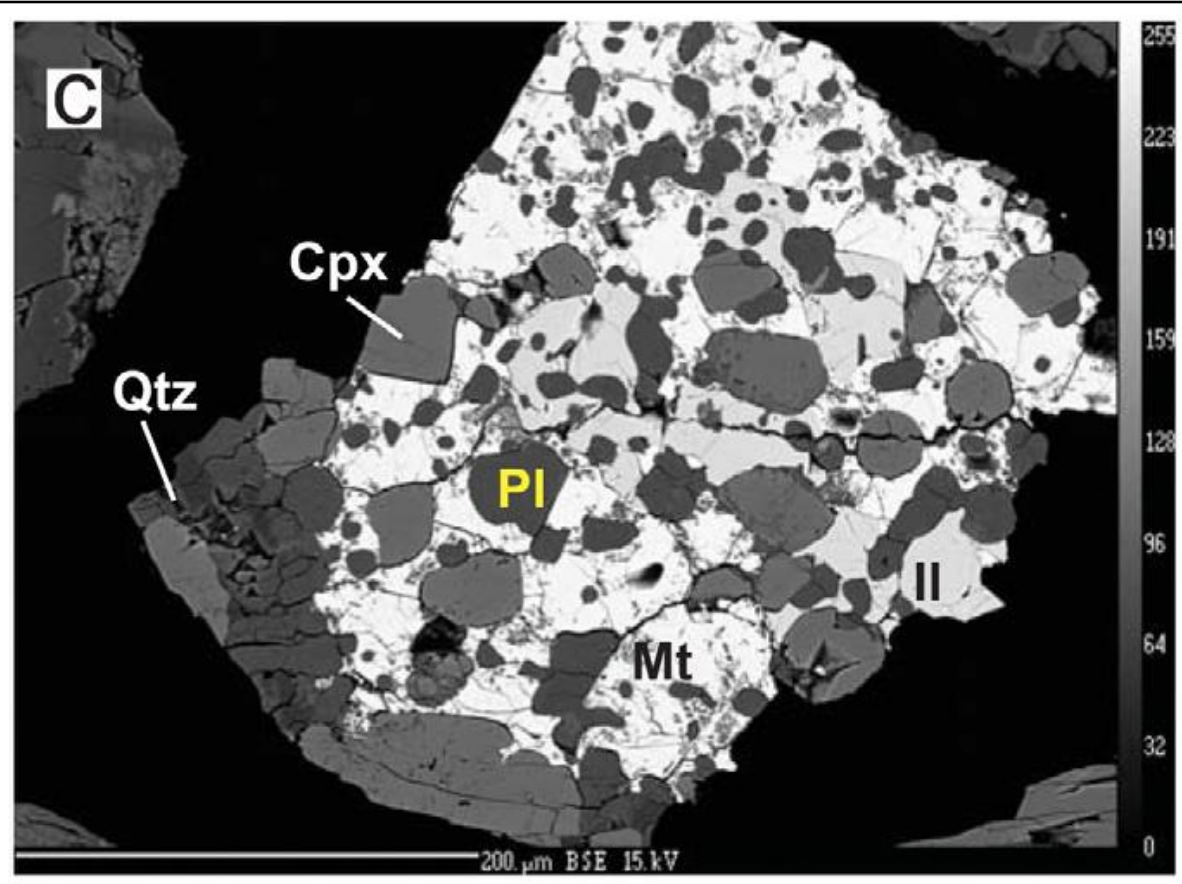

Figure 5 Backscattered electron image of a particle showing intergrown granoblastic phases in order of brightness (highest mean $\mathrm{Z}$ ). Magnetite (Mt), ilmenite (Il), clinopyroxene (Cpx), plagioclase (Pl), and quartz (Qtz). Black is epoxy.

Cathodoluminescence. Some insulators and semi-conductors produce UV and near-IR radiation (including visible light) when bombarded by the electron beam, due to the excitation and decay of electron-hole pairs in the target atom. The color and intensity of this radiation is extremely sensitive to trace element content and crystal lattice defects. Images of this radiation can be very useful in studies of growth zoning in minerals and other materials. Some electron microprobes are equipped to do cathodoluminescence (CL) imaging.

Secondary Electrons. Interactions in which energy is transferred from beam electrons to atoms in the sample (i.e. inelastic events) can cause loosely bound outer electrons of the sample 
atoms to gain enough energy that they can move through the sample. These secondary electrons have such low energy that only those produced very near the surface can escape the sample and be detected. Secondary electrons can be used to produce high-resolution images of the sample surface that are used in electron microscopy.

\section{Analytical Capabilities}

Sample Geometries. Three types of samples can be analyzed by electron microprobe: 1) bulk materials; 2) thin-film and stratified materials; and 3) particles. Bulk materials are the default sample configuration, these are typically flat, micro-polished surfaces (i.e. polished petrographic thin sections or bulk cast epoxy mounts), these must generally be no thinner than $\sim 25$ microns. The polished surface must be coated with carbon, a task usually accomplished just prior to analysis. Because the electron microprobe is very sensitive for surface analysis, specialized techniques exist for detecting thin surface layers down to monolayer thickness. This type of thin film analysis requires additional training and the use of special software designed for the purpose. Finally, particles with a grain size larger than 10 microns can be cast in epoxy and polished as for bulk samples. Particles smaller than 5 microns can be dispersed on double stick carbon tape and placed on a planchet, and analyzed using a rastered electron beam. This type of analysis requires additional corrections for the geometries of the sample.

Sample Requirements. Samples must be planar on a micron scale and polished to a 0.25-micron final polish. They must be either electrically conductive or coated with carbon (typically by using a high-vacuum carbon evaporator). The sample must also be stable in a high vacuum environment. Samples must be free from residual oils (i.e. hand oils), uncured epoxy, or other substances that will outgas and contaminate the microprobe. Common sample sizes include 1-inch rounds and standard $27 \mathrm{~mm}$ x $46 \mathrm{~mm}$ thin sections. A number of commercial laboratories exist for sample preparation, although radioactive samples do require special handling that many commercial laboratories are unable to accommodate.

\section{Strengths and weakness of the Electron Microprobe Techniques}

Strengths. Electron microprobe instruments are ideal for measuring in-situ elemental composition of solid samples. Backscattered electron imaging in combination with EDS allows for rapid characterization of the various phases present in a specimen, and can reveal elemental zoning and other phase relations that are otherwise undetectable by other techniques. Quantitative WDS measurements provide precise compositional data in a relatively timely manner, and are invaluable in studies of mineral equilibria. The microprobe can be used to produce elemental maps of specimens that can reveal heterogeneities.

Weakness. Although the volume of material sampled in the electron microprobe analysis is quite small, and is considered to be non-destructive, the preparation of thin sections or 
epoxy mounted probe rounds consumes a significant amount of material. Sample preparation can be time consuming, and difficult especially if the material is friable or radioactive. WDS measurements can be time consuming as well. Some mineral phases, including many carbonate phases, are sensitive to the heat produced by electron beam interactions and may decrepitate while under interrogation. 


\section{Transmission Electron Microscopy (TEM)}

\section{Introduction}

Since transmission electron microscope was invented in 1930s, it has been extensively developed and evolved into a versatile electron-optic instrument in combination with X-ray energy dispersive spectroscopy (EDS) and electron energy loss spectroscopy (EELS), especially, in the past 10 years, commercial aberration correction devices and monochromator further enhancing the instrument performances and making it become an even more powerful and comprehensive tool in microstructural characterization of materials on the scale down to subÅngström spatial resolution in imaging (below atomic level) and sub-eV energy resolution in EELS (comparable to synchrotron X-ray absorption spectroscopy (XAS)). Atom resolved chemical mapping can also be achieved using either EDS or EELS.

Transmission electron microscopy (TEM) is an experimental technique for microstructural characterization of materials (including a variety of natural and man-made inorganic and organic condensed substances such as metal, alloy, ceramics, semiconductor, minerals, polymer, composite, biological cells, virus, proteins nucleic acids, and bacteria, etc.). It is performed under a transmission electron microscope where a high-energy beam of electrons $(50-1,000 \mathrm{keV})$ is transmitted through an ultra-thin specimen (typically on the order of tens to $100 \mathrm{~nm})$ in a vacuum column $\left(10^{-4}\right.$ to $10^{-7} \mathrm{~Pa}$ or higher) after undergoing elastic and inelastic electron scatterings due to interactions between the beam and the specimen, from which a variety of physical signals is simultaneously generated. Each of the signals is reflecting of a certain information associated with the specimen, therefore, can be used in TEM for examining and measuring microstructures and material properties of the specimen, including topography, morphology, constitutive compound(s) or phase(s), crystal structure and crystallographic orientation, interfaces and crystal defects, chemical element composition, valence states and chemical bonding, electronic band structure, magnetic structure, etc.. The TEM is unique among materials characterization techniques used in the nuclear forensics is in that it enables essentially simultaneous examination of microstructural features at an extensive magnification ranging from 50x to $1,000,000 \mathrm{x}$ through a significantly high spatial-resolution (can achieve better than $50 \mathrm{pm}$ ) imaging and acquisition of chemical and crystallographic information from a very small region (down to the order of nanometer) of specimen.

\section{Instrumentation}

Transmission electron microscope has two different categories or models, i.e. conventional transmission electron microscope (CTEM) and scanning transmission electron microscope (STEM). Both CTEM and STEM form an image from the electrons that are transmitted through a thin specimen. A modern transmission electron microscope usually integrates both CTEM and STEM with optional Cs-corrector for each, and may also combine with EDS and EELS. It, therefore, not only has the capabilities of imaging and diffracting but also can perform EELS and EDS spectroscopy measurements with a small, focused electron beam. STEM mode also can be particularly useful for atomic resolution spectroscopies (X-ray, electron energy loss, etc.) from a localized volume of the specimen. The CTEM can also yield near atomic resolution spectroscopy using imaging energy loss filters. 


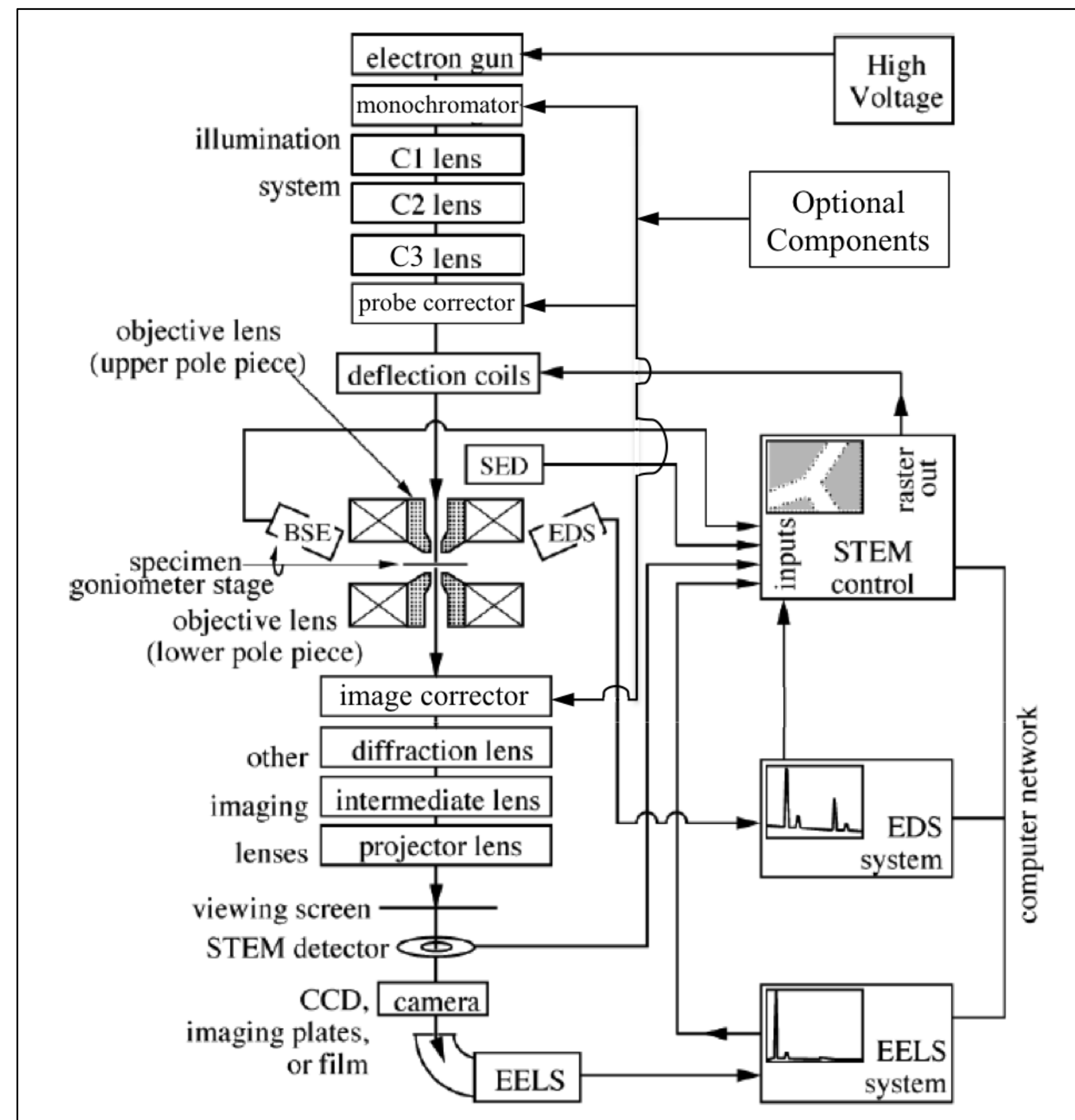

Figure 1. A block diagram of modern transmission electron microscope.

A block diagram of a modern transmission electron microscope is shown in Figure 1. The electrons emitted from the source (can be a Schottky-field emission gun (FEG), the SchottkyFEG combining with an optional monochromator, or a cold-FEG) are gathered and transfer by condenser lenses, and then passed through a probe Cs-corrector (if has) onto the specimen. With the exception of the electrostatic lens in the electron gun and the monochromator (Wien-filter) (i.e., electron source), lenses in the electron microscope are usually rotationally symmetrical magnetic lenses. The lenses in the Cs-correctors, however, are multi-pole (usually hexapole (6 
poles) or dodecapole (12 poles)) magnetic lenses. The condenser lenses can illuminate the specimen with a wide collimated parallel beam (in CTEM) or present the objective lens with a parallel narrow beam (in STEM). The objective lens images the specimen in CTEM mode or forms a small probe of atomic dimensions on the specimen in STEM mode. In the CTEM mode the objective forms a virtual image which is further magnified by several projector lenses and transferred onto the detector (usually a CCD) at the bottom of the Figure 1. The image detected by the CCD may be displayed on a monitor or computer. STEM and CTEM modes require the objective lens to be on opposite sides of the specimen. In a CTEM the whole image is formed (in parallel) at one time (Figure 2a) whereas in the STEM a focused probe is scanned across the specimen in a raster fashion and the image is sequentially built up, one pixel (or image point) at a time (Figure 2b). The STEM may have two (or more) different types of detectors. The bright field (BF) detector (on the optic axis) detects the electrons that have passed through the specimen without significant deviation. The annular dark field (ADF) detector can be used to detect the electrons that have been scattered to high angles (typically greater than three or four times the objective aperture angle), forming a high-angle annular dark-field (HAADF) (or "Z-contrast") image. This HAADF method uses incoherent elastic scattering of electrons to form images of atom columns.

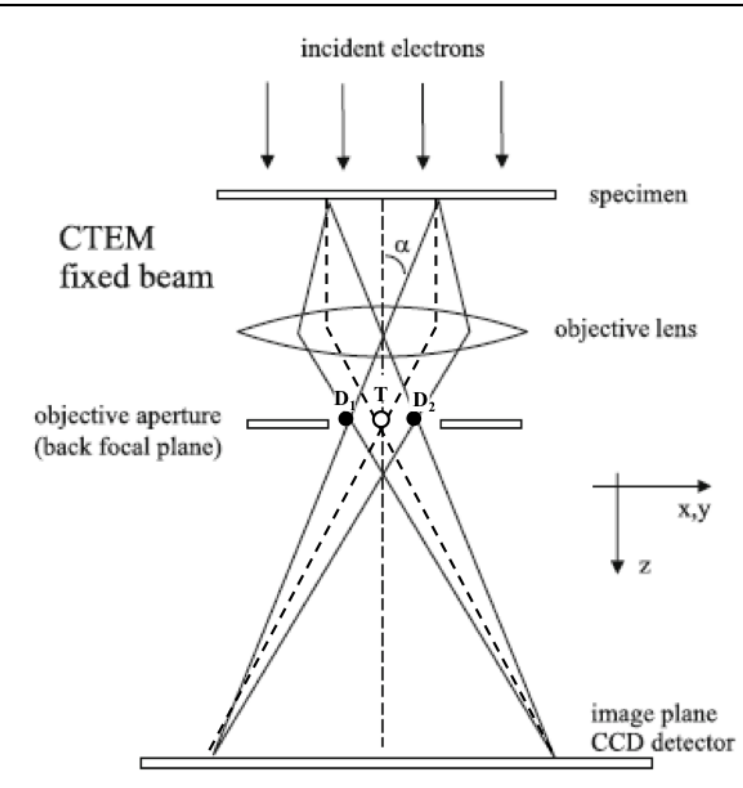

(a)

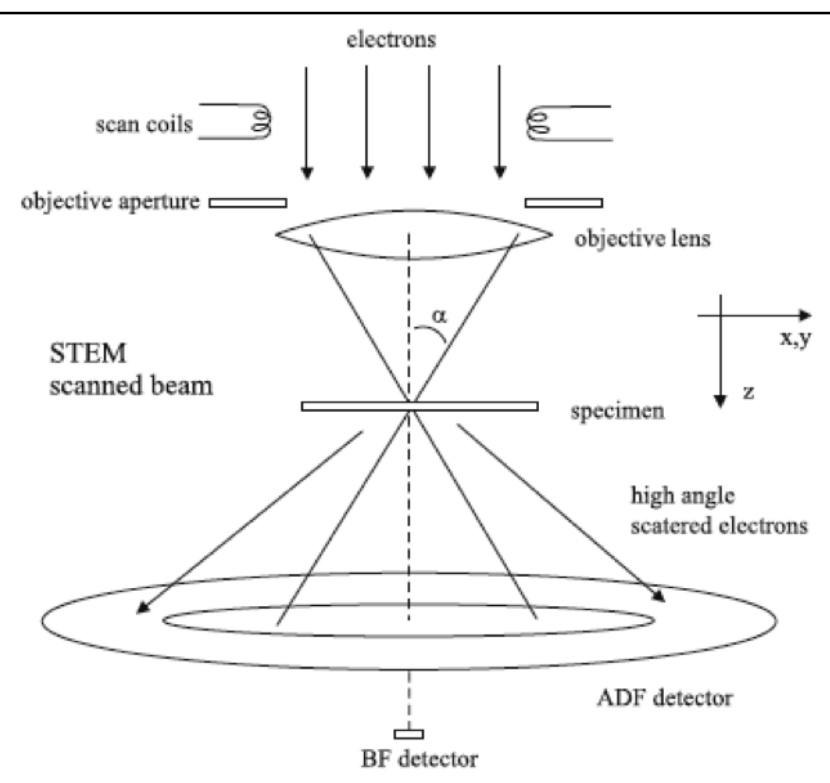

(b)

Figure 2. (a) Simplified model of a high-resolution conventional transmission microscope (CTEM). (b) Simplified model of a scanning transmission electron microscope (STEM). BF $=$ bright field, $\mathrm{ADF}=$ annular dark field.

\section{Sample requirements and preparation}

TEM specimens are required to be at most hundreds of nanometers thick. High quality samples will have a thickness that is comparable to the mean free path of the electrons that travel 
through the samples, which may be only a few tens of nanometers. Sample preparation, therefore, can be a complex procedure. Depending on the material under analysis and the desired information to obtain from the specimen, different technique will be used for the preparation of the required thin sections. The techniques often used include mechanical milling, ultramicrotome sectioning, chemical etching, ion etching, focused ion beam (FIB), etc.. The FIB is a relatively new technique to prepare thin samples for TEM examination. It can be used to prepare very thin membranes from a specific area of interest in a sample. FIB makes use of significantly more energetic gallium ions and may alter the composition or structure of the material through gallium implantation.

\section{Main functions and techniques}

Electron Diffraction. Electron diffraction is used in microstructural characterization of materials mainly for crystal structure determination, phase identification and crystallographic orientation measurement. In practice, the diffraction patterns measured by X-ray methods are more quantitative than electron diffraction patterns, but electrons have an important advantage over X-rays: electrons can be focused easily. By focusing the electron beam, diffraction patterns can be measured from microscopic regions, and it is often possible to select a single microcrystal for a diffraction measurement. The standard method for generating electron diffraction patterns using CTEM is by selected-area diffraction (SAD) in which selected-area aperture is applied to limit the area of specimen from which a diffraction pattern is obtained. Although the diameter of the selected-area aperture can be varied, but diffraction effects from the edge of the aperture limit the minimum area of the specimen from which a SAD pattern can be generated to a region approximately $0.5 \mu \mathrm{m}$ in diameter. Microdiffraction $(\mu-\mathrm{D})$ and convergent-beam electron diffraction (CBED) technique in either CTEM mode or STEM mode, however, allows the collection of diffraction data from areas of the specimen less than $0.5 \mu \mathrm{m}$ in diameter, and are very useful for structure determination of nanoparticle.

Spatial imaging. Imaging methods in CTEM mode utilize the information contained in the electron waves exiting from the sample to form an image. The electron diffraction pattern and the associated image are intimately related in that either the transmitted beam or any of the diffracted beams can be used to form the image. Bright-field imaging is commonly used for routine examination of microstructure features of a specimen such as defects, interfaces and second phase particles, which is produced with transmitted beam ("T" in Figure 2a) by applying the objective aperture allowing only the electron in the transmitted beam to pass through and contribute to the image. If we position the objective aperture to allow the electron in one of diffracted beams ("D" in Figure 2a) to pass through and contribute to the image, it is dark-field imaging. The images obtained by either the bright-field imaging or and the dark-field imaging are called "diffraction contrast" images because they display variations in the intensity of electron diffraction across a thin specimen. High-resolution transmission electron microscopy (HRTEM or HREM) imaging is demonstrated to allow the electrons in multiple beams including the transmitted beam to go through the objective aperture (Figure 2a) (or no objective aperture applied) and form the image. In the HRTEM imaging process, the phase of the diffracted electron wave is preserved and interferes constructively or destructively with the phase of the transmitted wave, therefore, the HRTEM image is called "phase-contrast" image that allows the 
imaging of the crystal structure of a specimen at an atomic scale. Because of its high resolution, it is a valuable technique to study nanoscale properties of crystalline materials.

Imaging in the STEM mode also allows for generation of bright-field and dark-field images. These images contain the same information contained in their CTEM analogs. Atomic resolution images of which the contrast is directly related to the atomic number (Z-contrast image) are also achievable using high-angle annular dark-field (HAADF) imaging. The rastering of the beam across the specimen makes the microscope suitable for analysis techniques such as mapping by EDS, EELS and ADF. These signals can be obtained simultaneously, allowing direct correlation of image and quantitative data.

EDS and EELS. Besides diffraction and spatial imaging, the high-energy electrons in CTEM and STEM cause electronic excitations of the atoms in the specimen. Two types of spectrometries (EDS and EELS) can be used to obtain chemical information from electronic excitations.

In EDS, an X-ray spectrum is acquired usually using a solid-state detector from small regions of the specimen illuminated with a focused electron beam. Characteristic X-rays from the chemical elements are used to determine the concentrations of the different elements in the specimen. EDS is particularly sensitive to heavier elements.

In EELS, energy losses of the electrons are measured after the high-energy electrons have traversed the specimen. Information on local chemistry and structure is obtained from features in EELS spectra caused by plasmon excitations and core electron excitations. EELS is capable of measuring atomic composition, chemical bonding, valence and conduction band electronic properties, surface properties, and element-specific pair distance distribution functions. EELS tends to work best at relatively low atomic numbers, where the excitation edges tend to be sharp, well-defined, and at experimentally accessible energy losses (the signal being very weak beyond about $3 \mathrm{keV}$ energy loss).

\section{Other techniques}

Besides the main techniques above it is possible to perform many other experiments in a modern transmission electron microscope.

Energy-filtered transmission electron microscopy (EFTEM). EFTEM is a technique used in CTEM, in which only electrons of particular kinetic energies are used to form the image or diffraction pattern. The technique can be used to aid chemical analysis of the sample in conjunction with complementary techniques such as electron crystallography. Improved elemental maps can be obtained by taking a series of images, allowing quantitative analysis and improved accuracy of mapping where more than one element is involved. By taking a series of images, it is also possible to extract the EELS profile from particular features.

Lorentz microscopy. Specialized adjustments of lens currents permit imaging of magnetic structures such as domain walls (interfaces where the direction of magnetization 
changes by a large angle). When the electrons pass through a magnetic specimen, they are deflected slightly by Lorentz forces, which change direction across a domain wall.

Holography. Electron holography is a fascinating technique, although it is by no means a standard tool for materials characterization. It is a method for making images without the use of lenses, so images made by electron holography use the CTEM in an unconventional way. The CTEM is modified with the addition of an electron biprism, utilizing electric fields around a fine wire of micron diameter. The electron wavefunction can pass to either side of the wire, and the self-interference between the two sides produces a set of fringes on the viewing screen. A sample, placed to one side of the wire, distorts the phase of the electron wavefunction, so the self-interference pattern is modified. There is a substantial phase shift of the electron wavefunction even when the sample is very thin - thickness variations of atomic size can be detected. Electron holography also demonstrates a remarkable quantum behavior, the AharonovBohm effect, which proves that the vector potential is more fundamental than the magnetic field because the phase of the electron wavefunction is altered by the vector potential even if the electron does not pass through a region of magnetic field.

Electron Tomography: As TEM specimen holders typically allow for the rotation of a sample by a desired angle, multiple views of the same specimen can be obtained by rotating the angle of the sample along an axis perpendicular to the beam. By taking multiple images of a single TEM sample at differing angles, typically in $1^{\circ}$ increments, a set of images known as a "tilt series" can be collected. Under purely absorption contrast conditions, this set of images can be used to construct a three-dimensional representation of the sample.

\section{Strengths and weakness of the TEM technique}

Strengths. The TEM is unique among materials characterization techniques is in that it enables essentially simultaneous examination of microstructural features at an extensive magnification ranging from $50 \mathrm{x}$ to $1,000,000 \mathrm{x}$ through a significantly high spatial-resolution (can achieve better than $50 \mathrm{pm}$ ) imaging and acquisition of chemical and crystallographic information from a very small region (down to the order of nanometer) of specimen.

Weakness. Electron microscopes are expensive to build and maintain. Many materials require extensive sample preparation to produce a sample thin enough to be electron transparent, which makes TEM analysis a relatively time consuming process with a low throughput of samples. The field of view is relatively small, raising the possibility that the region analyzed may not be characteristic of the whole sample. There is potential that the sample may be damaged by the electron beam, particularly in the case of biological materials. 


\section{X-Ray Diffraction}

\section{Introduction}

X-ray diffraction (XRD) is a non-destructive technique used to obtain compositional and structural information on crystalline and semi-crystalline materials. XRD can provide information on the composition, concentration and purity of a multi-component crystalline system, the crystallographic nature of the materials, as well as the crystallite (grain) size.

\section{History of X-ray diffraction}

Wilhelm Conrad Roentgen accidentally discovered "X-radiation" in 1895. In 1912, Max von Laue used X-rays in the first diffraction experiment and determined that X-rays have a wavelength similar to the interatomic distances in crystals. He was able to demonstrate in a later experiment that if the interatomic distances in the crystal are known then the wavelength of the X-rays can be measured, or alternatively, if the wavelength is known, diffraction experiments can be used to determine the interplanar spacings of a crystal. In 1913 William H. Bragg and his son William L. Bragg determined the first mineral structure $(\mathrm{NaCl})$ from $\mathrm{XRD}$, and also determined the atomic-level structure of many more minerals. They also simplified von Laue's mathematical generalization and introduced the Bragg equation, $\lambda=2 \mathrm{~d} \sin \theta$, which describes the condition for diffraction to occur in terms of the wavelength of the x-radiation $(\lambda)$, the interplanar ("d") spacings of the crystal, and the angle of incidence of the radiation with respect to the crystal planes $(\theta)$. Advances in XRD since Bragg's time has occurred in instrumentation.

\section{The Electromagnetic Spectrum, generation of X-rays, and the Braggs' Equation}

X-rays can be thought of as waves with wavelengths on the order of $0.1 \AA$ to $\sim 10 \AA$; the shorter the wavelength, the more energetic the wave. Because of the relatively short wavelengths of electromagnetic radiation in the $\mathrm{X}$-ray region, $\mathrm{X}$-rays are high energy waves and are much more penetrating compared to UV, visible, IR, or radio waves. When electrons strike a metal anode with sufficient energy, X-rays are produced. This process is typically accomplished using a

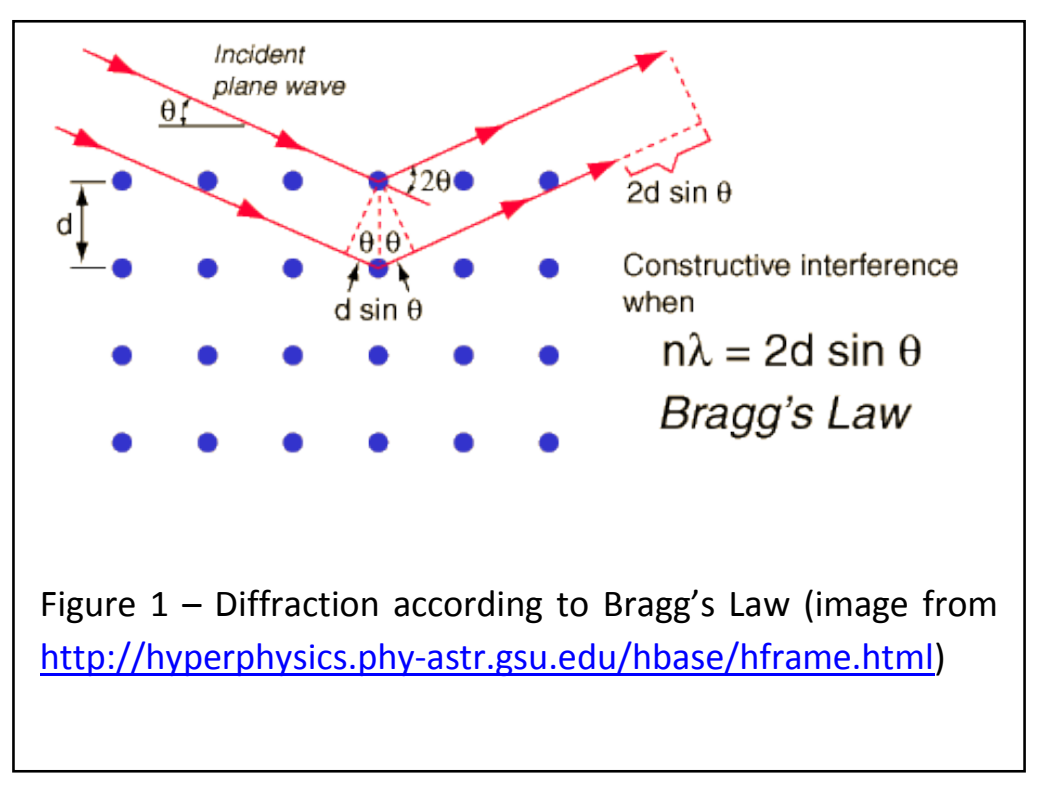


sealed x-ray tube, which consists of a metal target (often copper metal) and a tungsten metal filament, which can be heated by passing a current through it (typically 10-15 mA), resulting in the "boiling off' of electrons from the hot tungsten metal surface. The collision between these energetic electrons and electrons in the target atoms results in electron from target atoms being excited out of their core-level orbitals, placing the atom in a short-lived excited state. The atom returns to its ground state by having electrons from lower binding energy levels (i.e. levels further from the nucleus) make transitions to the empty core levels. The difference in energy between these lower and higher binding energy levels is radiated in the form of X-rays. Thus Xrays provide a convenient means of determining what elements are present in a sample because of the unique wavelengths produced by each unique element. Crystals are ordered, threedimensional arrangements of atoms with characteristic periodicities. As the spacing between atoms is on the same order as X-ray wavelengths (1-3 $\AA$ ), crystals can diffract the radiation when the diffracted beams are in-phase. The Bragg equation is given as $\mathbf{n} \boldsymbol{\lambda}=\mathbf{2 d \boldsymbol { d }} \boldsymbol{\operatorname { s i n } \boldsymbol { \theta }}$, and the modified Bragg equation is $\mathbf{d}=\boldsymbol{\lambda} / \mathbf{2} \sin \boldsymbol{\theta}$. For a given wavelength $(\lambda)$, diffraction can only occur at a certain angle $(\theta)$ for a given $\mathrm{d}$-spacing (Figure 1 ).

\section{Instrumentation}

X-ray diffractometers (single crystal and powder instruments) are manufactured by companies such as Bruker AXS, PANalytical, Rigaku, and others. Portable XRD's for field applications are available as well. Many of the commercially available instruments have similar designs and roughly equivalent capabilities. Manufacturers offer instruments that can be uniquely adapted to the needs of the customer with different add-ons such as capillary holders for liquid samples, heated stages for real time phase transformation studies, different detectors, and high throughput automatic sample changers. Figure 2 shows an example of a Bruker AXS powder X-ray Diffractometer.

Figure 3 shows the specifics of the BraggBrentano goniometer setup also visible in Figure 2. Here the X-ray tube shown on the left generates X-rays that pass through a collimator or divergence slit, hits the sample which is loaded horizontally, and are reflected into the detector after passing through another collimator or antiscatter slit. Data is often

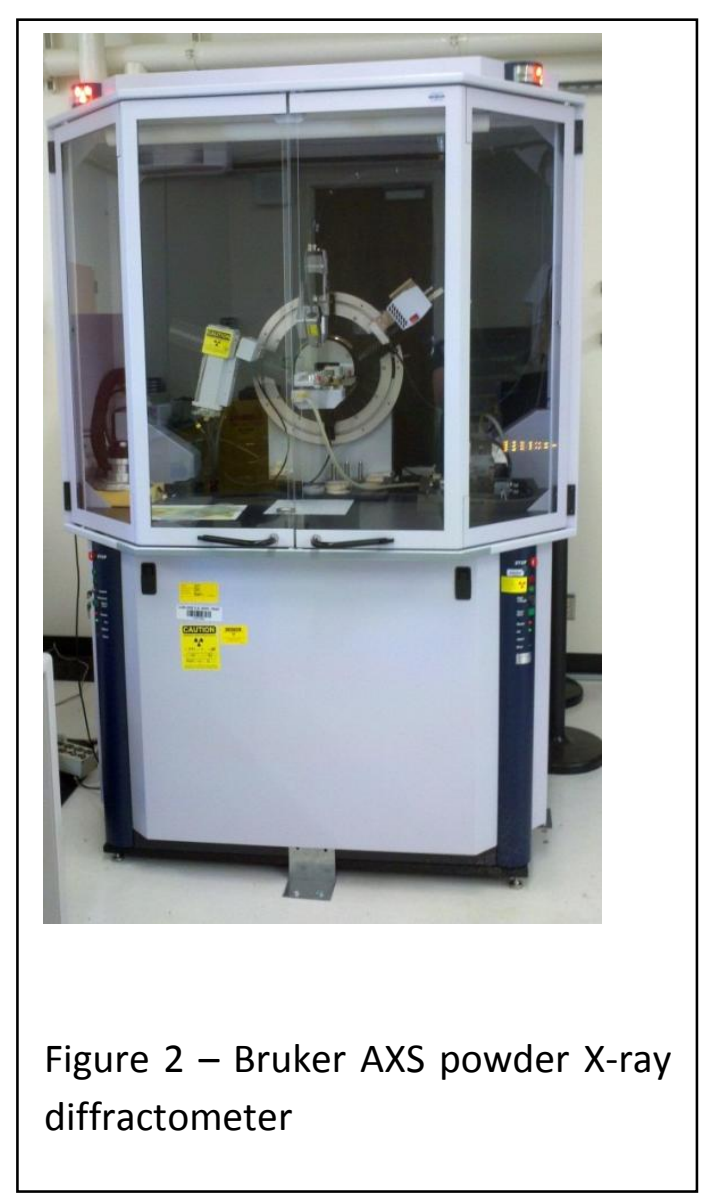


collected as counts/second. As mentioned previously, there are many variations of slit and detector settings that can be fine tuned for specific sample types.

\section{Types of samples commonly analyzed using XRD}

Powder samples - a powder is used to ensure completely random crystal orientation allowing for diffraction from all possible planes. X-rays produced in a tube are directed at a sample, and by virtue of the atomic arrangements in the sample, the $\mathrm{x}$-ray is diffracted at various discrete angles. The scattering of x-rays by crystal atoms produces a diffraction pattern that yields information about the structure of the crystal. Spectra are obtained by acquiring the x-rays intensity as a function of scattering angle. In general, each crystallographic atomic arrangement has a unique set of specific number of peaks defined by their intensities and angular positions. Powder samples can be rotated during analysis to ensure random collection of reflected $\mathrm{x}$-rays. An

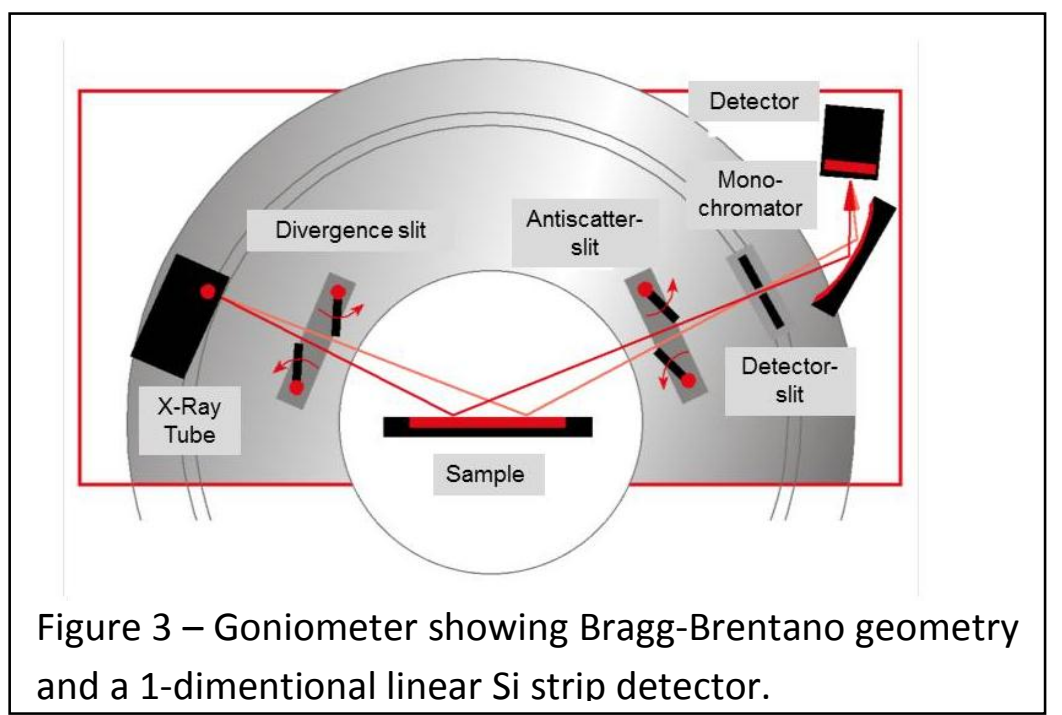

described next. example of powder spectra is shown in Figure 4 with intensity on the $y$-axis and scan angle in $2 \theta$ on the $\mathrm{x}$-axis.

Single crystals - a beam of $\mathrm{X}$ rays is directed at a single crystal, which sits stationary in front of a detector. The resulting data shows diffraction from a given series of atomic planes. Single crystal $\mathrm{x}$-ray is more helpful in determining crystal structure than powder diffraction

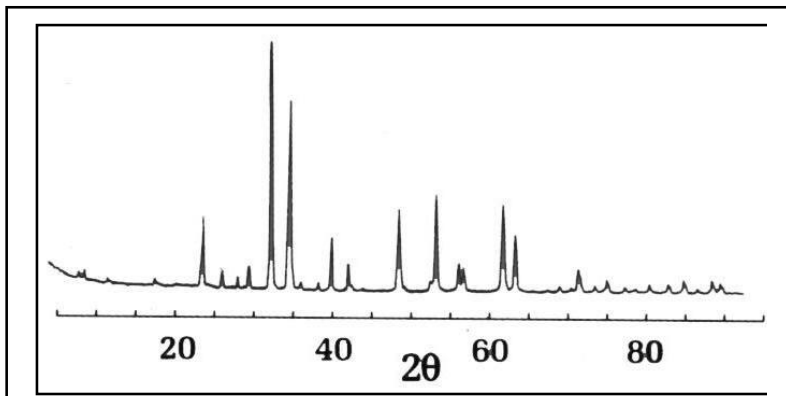

Figure $4-X$-ray spectra plot
Solids - samples are oriented in holders so that the X-ray beam penetrates the top layer of the sample. Solid samples are usually rotated during analysis to ensure random collection of reflected x-rays.

Liquids - samples are sealed in capillary tubes, and rotated during analysis. Structure information of the atoms can be gained by x-ray diffraction of liquids. 


\section{Data analysis}

All commercially available XRD instruments are equipped with data analysis software. The different search/match software packages utilize databases compiled and organized by the International Centre for Diffraction Data (ICDD). These databases contain hundreds of thousands of patterns to aid in phase and structure identification, and new patterns are being added all the time. There are also many different open source software packages that are available. Search/match programs allow users to do searches randomly (searching entire database) or adding in data acquired by other analyses to narrow the field of possible matches.

\section{Strengths and weakness of X-ray Diffraction}

\section{Strengths}

$\mathrm{X}$-ray diffraction is a quick and easy technique used to obtain compositional and structural information on crystalline and semi-crystalline materials. Relative proportions of mineral mixtures can be obtained by comparing diffraction line intensity. An advantage is that it is a non-destructive technique, allowing for samples to be used for other analyses.

\section{Weakness}

XRD works best with crystalline materials which makes glasses and partially crystalline materials problematic to identify using this method. Phases of less than a few wt. \% are also difficult to identify by XRD, however as noted above, identification of minor phases can be aided by trace element solution data (by ICP-MS) as well as compositional phases (by X-Ray Fluorescence). Phase identification in scans containing many peaks can be problematic also if nothing is known about the sample, and search match results might indicate unlikely matches. Analysts must rely on previous knowledge of similar scans to best determine phase matches.

\section{Conclusions}

$\mathrm{XRD}$ is a powerful determinative method in the mineral, materials, and forensics sciences. There are some limitations, but X-ray diffraction can also be a very straightforward and easy to use method to determine the identity of minerals or chemical phases. 


\section{Secondary Ion Mass Spectrometry}

\section{Introduction}

Secondary Ion Mass Spectrometry (SIMS) is used for elemental and isotopic analysis of solid samples. SIMS is a destructive technique, although the amount of sample consumed during analysis is usually quite small. The greatest strength of SIMS is the ability to analyze very small areas (as small as $50 \mathrm{~nm}$ using the CAMECA NanoSIMS, for example) and to generate highspatial resolution maps of the distribution of elements and isotopes within the sample. It can determine the abundances of elements that are present at trace levels, as well as stable light isotopic ratios, such as $\mathrm{O}$ and $\mathrm{S}$, and in some cases ratios of heavy actinide isotopes. SIMS is a surface sensitive technique, since the secondary ions at any point in the analysis derive from the

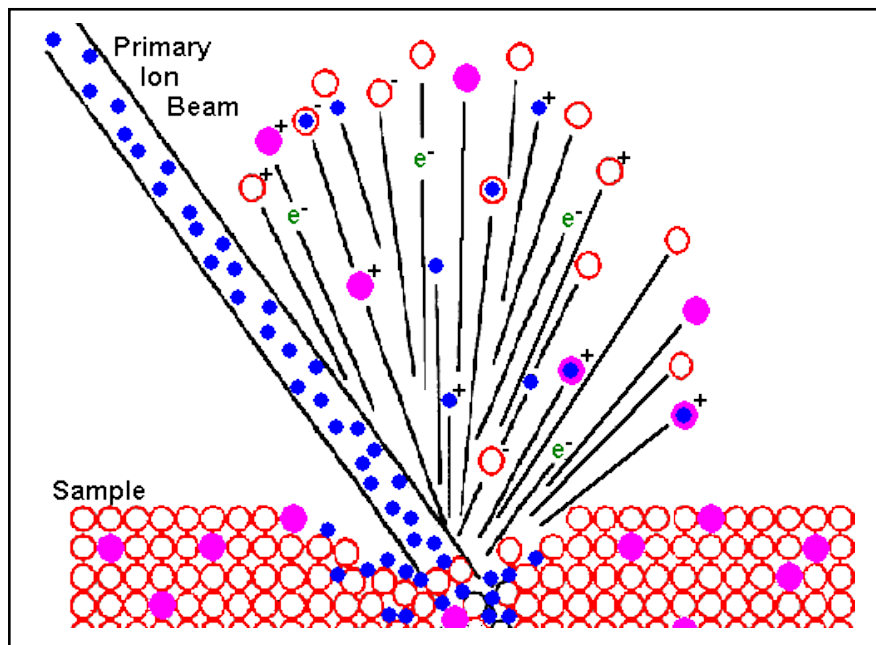

Figure 10. Schematic of the sputtering process in SIMS. top 3-5 monolayers of material (Figure 10). However, repetitive analyses can be performed over longer periods as the primary ion beam sputters away the sample (to a depth of several microns in some instances) in a technique known as "depth profiling." The measurement of the isotopic composition of sample is usually straightforward, only requiring the analysis of the sample and that of an isotopic reference material for determination of the mass bias of the instrument. Quantification of elements, however, involves the analysis of matrix matched standards for the determination of the relative sensitivity factor (a function of both the element to be analyzed and the matrix). SIMS is commonly used in nuclear forensics for exploring the heterogeneity of the material on fine spatial scale.

\section{Instrumentation}

A major manufacturer of ion microprobes is CAMECA. They manufacture three general types of instruments that are often used for nuclear forensic measurements (Figure 11). These include the "xf"-series instruments which are double-focusing mass spectrometers. These instruments have one or two Faraday collectors and a single electron multiplier suitable for stable isotope measurements and trace element determinations.

The second type of instrument is the IMS 1280-HR. This instrument is designed to complete high precision isotope ratio measurements. The CAMECA ims-1280 has similar ion optics to the "xf" series of instruments (Figure 12), but with a much larger geometry. The use of such large geometry instruments is often termed "LG SIMS." The larger geometry of the mass 


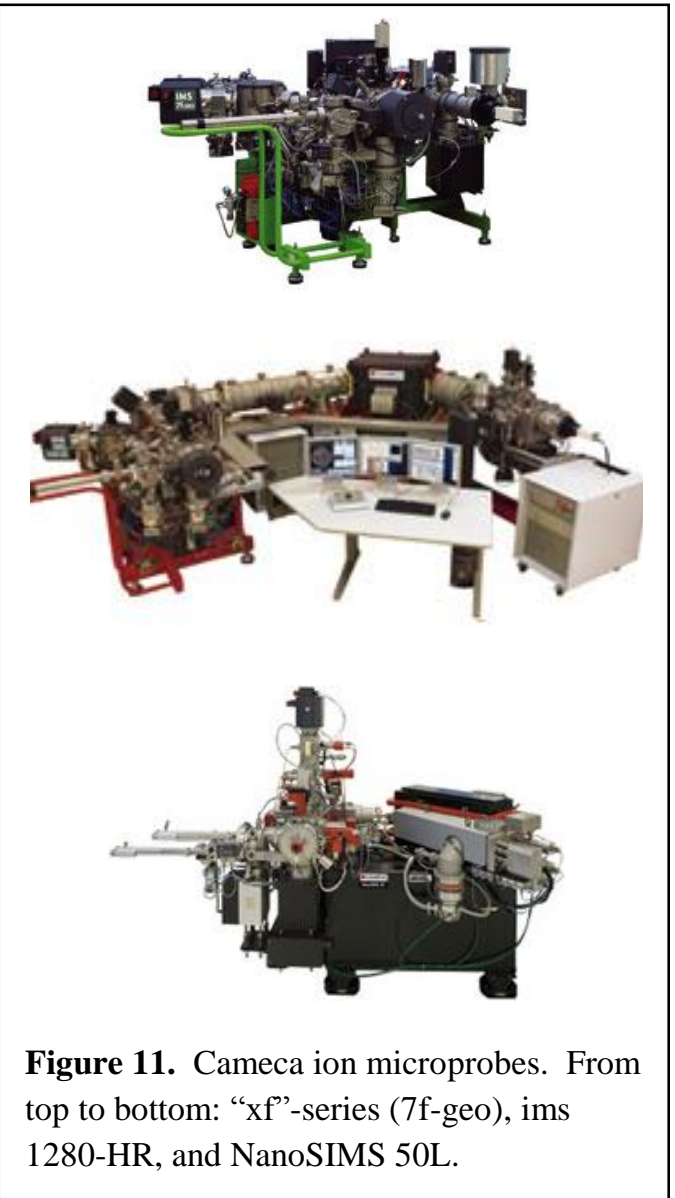

spectrometer affords increased transmission at higher mass resolving powers, allowing the reduction of interferences without a concomitant loss in sensitivity. The IMS 1280-HR has a multi-collector system composed of multiple faraday collectors and secondary electron multipliers. Like the " $x \mathrm{f}$ "-series instruments it is used to determine stable isotope compositions in materials requiring high spatial resolution. However, it is also commonly used for precise heavy isotope ratio measurements such as actinide isotope ratio measurements in nuclear particles.

The final instrument type made by CAMECA is the NanoSIMS 50L. The CAMECA NanoSIMS 50 is a uniquely designed SIMS instrument that offers extremely high spatial resolution $\left(50 \mathrm{~nm}\right.$ for $\mathrm{Cs}^{+}$ primary ion bombardment; $\sim 150 \mathrm{~nm}$ for $\mathrm{O}^{-}$ bombardment), but also with excellent sensitivity. This instrument has significantly higher spatial resolution than the other instruments, but lacks the high mass resolution of the IMS 1280-HR. The smaller analysis volume of the NanoSIMS 50L generally results in analysis of fewer atoms than the ims series instruments and therefore has lower precision. This instrument is used in nuclear forensics for analysis of light stable isotopes in nuclear materials.

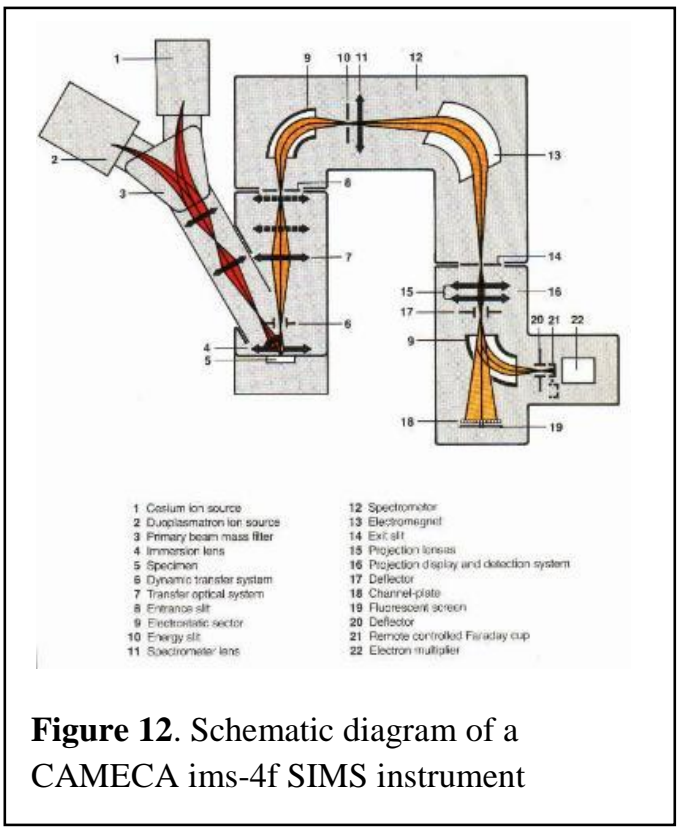

Mass Analyzers. Different commercial SIMS instruments use different mass analyzers, including double-focusing, quadrupole, and time-of-flight mass analyzers. The CAMECA ims-series SIMS instruments, for example, use a double-focusing mass spectrometer, consisting of a $90^{\circ}$ spherical electrostatic sector and a $90^{\circ}$ stigmatically focusing magnetic sector to separate the secondary ions according to their mass-to-charge ratio (Figure 12). Since most ions are singly charged, the mass spectrometer separates the secondary ions effectively according to their mass. Double focusing gives these instruments the capability for moderately high mass resolving power (ability to separate closely spaced masses). The mass resolving power $(\mathrm{M} / \Delta \mathrm{M})$ on a 
CAMECA $3 \mathrm{f}-7 \mathrm{f}$ instruments, for example, is variable from approximately 300 to 20,000 , depending on the instrument settings.

Detectors. The intensity of the mass-resolved ion beam is measured either with a Faraday cup, ion multiplier, or position-sensitive detector. The Faraday cup captures the ion beam, allowing the direct measurement of the resulting current. The ion multiplier converts ion impacts into electrical pulses, which are then counted for a specific period of time to yield a count rate (ions per unit time). The position-sensitive detector simultaneously counts ion impacts and measures their position of impact on the detector surface.

Imaging. In addition, most of the CAMECA SIMS instruments are stigmatically focusing, in which the spatial position of ions as they leave the sample surface is maintained and magnified through the instrument. Stigmatic focusing allows the real-time visualization of massresolved images through the use of a position-sensitive detector, such as a phosphor screen or resistive anode encoder. This mode of imaging is called "direct" or "microscope" ion imaging. Mass-resolved images can also be reconstructed from the secondary ion intensity information acquired while rastering a finely focused primary ion beam over the surface of the sample. This mode of imaging is called "microprobe" or "rastered" ion imaging.

\section{Application}

Bulk analyses assume that the material analyzed is homogeneous and can be represented by a single concentration or isotopic ratio without loss of information. SIMS can be used to measure isotopic and elemental abundances in bulk samples, but inductively coupled plasma mass spectrometry (ICP-MS) is usually more sensitive and accurate/precise for bulk measurements than SIMS. Even so, SIMS may still be appropriate for special cases, e.g., the analysis of $\mathrm{Si}$ when $\mathrm{HF}$ has been used during the sample dissolution process. SIMS may also be the technique of choice for electro-negative elements $(\mathrm{C}, \mathrm{N}, \mathrm{O}, \mathrm{F}, \mathrm{Cl}, \mathrm{S})$ for which ICPMS has poor sensitivity.

In many cases, however, the nuclear material will be heterogeneous. This heterogeneity may convey information about either the source of the material or the process used to make it. Because of the small size of the primary ion beam, SIMS can be used to measure isotopic and elemental abundances as a function of lateral $(\mathrm{X} \& \mathrm{Y})$ position. The spot size of a SIMS primary ion beam is usually around $1 \mu \mathrm{m}$, but can be as small as $50 \mathrm{~nm}$ for the CAMECA NanoSIMS 50 . Plots of isotopic or elemental abundances versus lateral position are called "images" or "maps." In addition, SIMS can be used to measure isotopic and elemental abundances, as a function of time. If the sputter rate remains constant during the analysis, or varies in a known way, the variation of isotopic and elemental abundances as a function of depth (Z) can be calculated. Plots of isotopic or elemental abundances versus depth are called "depth profiles." 


\section{Sample requirements}

SIMS can only be used to analyze solid samples. SIMS samples must either conform dimensionally to the instrument's sample holder or may be cut to fit. Small samples, such as particles, can be mounted onto an appropriate substrate that fits into the instrument's sample holder. SIMS is a destructive analysis technique, although the amount of material removed during most analyses is quite small.

\section{Strengths and weakness of the SIMS technique}

Strengths. One of the key advantages of SIMS is the ability to measure both elements and isotopes with high sensitivity. Detection limits vary with the element of interest and the matrix, but typically range from 1 ppba to 1 ppma. Under optimum conditions, elemental concentrations from SIMS data can be obtained with $+/-2 \%$ precision and $+/-10 \%$ accuracy. The precision of isotopic ratios from SIMS data is often limited by counting statistics and can approach $0.1 \%$ or better. With proper calibration of the mass bias of the instrument, the accuracy of isotopic ratios from SIMS data is comparable to the precision.

Weakness. SIMS analysis can be affected by isobaric interferences, such as ionic species so close in mass to the species of interest that the mass spectrometer cannot separate them. In addition to interferences from atomic ions of the same mass, e.g., ${ }^{40} \mathrm{~K}^{+}$and ${ }^{40} \mathrm{Ar}^{+}$in the measurement of ${ }^{40} \mathrm{Ca}^{+}$, the analysis can also suffer from interferences from molecular ions of the same nominal mass, e.g. ${ }^{28} \mathrm{Si}^{16} \mathrm{O}^{+}$and ${ }^{12} \mathrm{C}_{3} \mathrm{H}_{8}{ }^{+}$in the measurement of ${ }^{44} \mathrm{Ca}^{+}$. These molecular ions can be formed from constituents in the sample itself (usually matrix level constituents), the sample vacuum, and the primary ion beam. Interferences can be reduced to some extent by instrumental improvements, such as mass filtering of the primary ion beam and improved sample chamber vacuum. 


\section{Modified Davies and Gray Technique for Uranium Assay}

\section{Introduction}

The Modified Davies and Gray (Davies-Gray) technique is an analytical method for determining the concentration of uranium in solution by electrochemical titration. It is a useful complementary technique to the measurement of uranium concentration by isotope dilution mass spectrometry (IDMS) as it is faster and requires considerably less infrastructure, but lacks the precision and dynamic range of IDMS. The Davies-Gray technique is particularly useful for measuring samples with high uranium concentrations, such as uranium ore concentrates, uranium fuels, and liquid samples contaminated with uranium.

\section{Basic Theory}

The Davies-Gray technique is an electrochemical titration technique described in detail in ASTM C1267 - 06. Uranium in solution, present as U(IV) and U(VI), is quantitatively reduced to U(IV) by adding a solution of $\mathrm{Fe}$ (II) in phosphoric acid and sulfamic acid to excess. Excess Fe(II) is oxidized to $\mathrm{Fe}(\mathrm{III})$ by adding a solution of $50 \%$ nitric acid with a $\mathrm{Mo}$ (VI) catalyst. A solution containing $\mathrm{V}(\mathrm{IV})$ is added to improve the titration reaction rate, and $\mathrm{U}(\mathrm{IV})$ is titrated to a potentiometric end-point with a solution containing $\mathrm{Cr}(\mathrm{VI})$. The concentration of uranium is determined based on the mass of the $\mathrm{Cr}(\mathrm{VI})$ solution (calibrated using a uranium solution standard or a standard $\mathrm{Cr}(\mathrm{VI})$ solution) used to titrate the mass of the sample to the end-point.

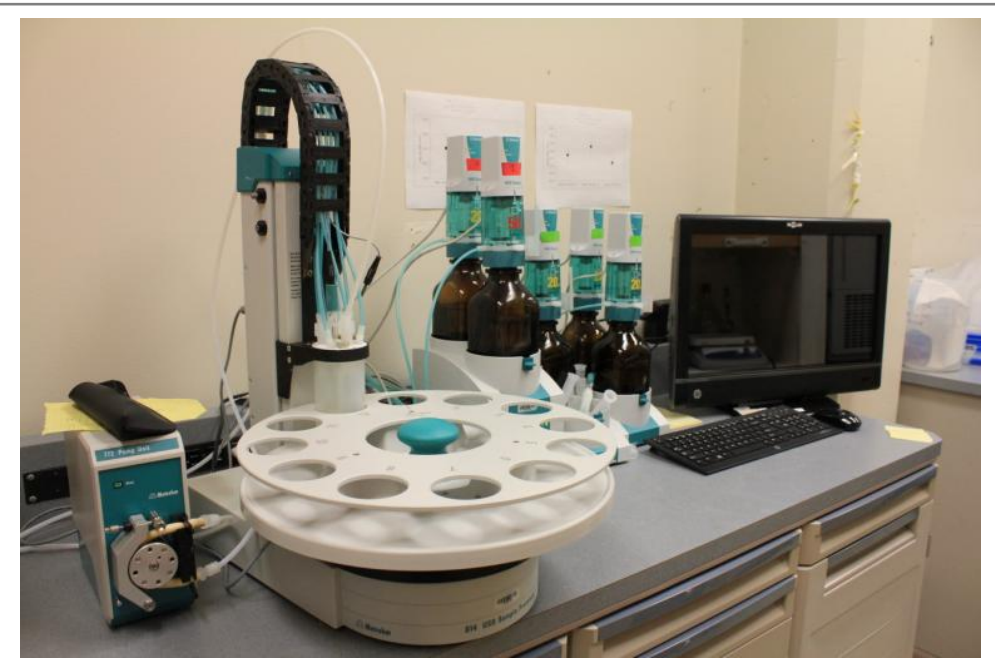

Figure 1. Auto-titration system (Metrohm) at Lawrence Livermore National Laboratory.

\section{Instrumentation}

Individual Davies-Gray analyses can be performed by hand, although this approach is time-consuming and difficult to reproduce to the precision required for useful results. Routine application of the DaviesGray technique is greatly aided by the use of an autotitration apparatus (Figure 1). Inexpensive and highly adaptable set of instrumentation is available 
from many manufacturers. Although the initial setup and fine-tuning of the apparatus is trial-anderror and requires some effort, considerable time can be saved in the long run.

Auto-titration apparatus are programmable using software provided by the manufacturer. The software should be used to set up the method following the procedure described by ASTM C1267 - 06. Care should be taken to determine the desired volume of reagent to add at each step. The potentiometric end-point can be set, and some fine-tuning may be required to program the software to accurately identify a single end-point in a reproducible fashion. The titration of $\mathrm{U}(\mathrm{IV})$ with $\mathrm{Cr}(\mathrm{VI})$ is the most sensitive step in the procedure, and the software should be programmed to add incrementally smaller volumes of the $\mathrm{Cr}(\mathrm{VI})$ solution as the end-point is approached.

The following reagents should be prepared in order to successfully perform the procedure:

1. Ferrous sulfate heptahydrate, $1.0 \mathrm{M}\left(\mathrm{FeSO}_{4} \square 7 \mathrm{H}_{2} \mathrm{O}\right)+\mathrm{H}_{2} \mathrm{SO}_{4}+\mathrm{H}_{3} \mathrm{PO}_{4}$

2. Sulfamic acid, $0.15 \mathrm{M}\left(\mathrm{NH}_{2} \mathrm{SO}_{3} \mathrm{H}\right)$

3. Potassium dichromate, $0.0045 \mathrm{M}\left(\mathrm{K}_{2} \mathrm{Cr}_{2} \mathrm{O}_{7}\right)$

4. Vanadyl sulfate dihydrate, $0.0038 \mathrm{M}\left(\mathrm{VOSO}_{4} \square \square 2 \mathrm{H}_{2} \mathrm{O}\right)+\mathrm{H}_{2} \mathrm{SO}_{4}$

5. Ammonium molybdate, $0.4 \%\left(\left(\mathrm{NH}_{4}\right)_{6} \mathrm{Mo}_{7} \mathrm{O}_{24} \square 4 \mathrm{H}_{2} \mathrm{O}\right)+\mathrm{NH}_{2} \mathrm{SO}_{3} \mathrm{H}+\mathrm{HNO}_{3}$

See ASTM C1267 - 06 for more specific directions on preparing the reagents.

\section{Analytical Issues}

Prior to performing the Davies-Gray technique, solid samples must first be dissolved using another method, and liquid samples should be dried down. Dried samples should be dissolved in $2 \mathrm{~mL} 1 \mathrm{M} \mathrm{HNO}_{3}$ prior to analysis. Ideally, an aliquot of the sample containing about $50 \mathrm{mg}$ total $\mathrm{U}$ should be used. Empirical evidence has shown that this method can tolerate uranium masses of 25 to $75 \mathrm{mg}$. However, it is possible to measure higher and lower concentrations of uranium if reagent concentrations are adjusted accordingly. Reagents should be prepared immediately before use whenever possible. The Fe and $\mathrm{V}$ solutions are particularly sensitive, as it is imperative that the valence states of Fe and V start at (II) and (IV), respectively. The addition of sulfuric acid will increase the temperature of the Fe and V solutions. They should be allowed to cool to room temperature before the crystalline Fe and $\mathrm{V}$ are added.

If an auto-titration apparatus is used, care must be taken to ensure that the titration procedure functions reliably. Jammed stir bars, improperly loaded samples, and other malfunctions can occur, and such events should be identified in order to exclude data obtained from an erroneous procedure. It is usually possible to identify failed titration runs on the basis of failure of autotitration software to identify the potentiometric end-point. Samples should be run in triplicate whenever possible to ensure accurate results (Figure 2), and results from failed titrations should be excluded. 

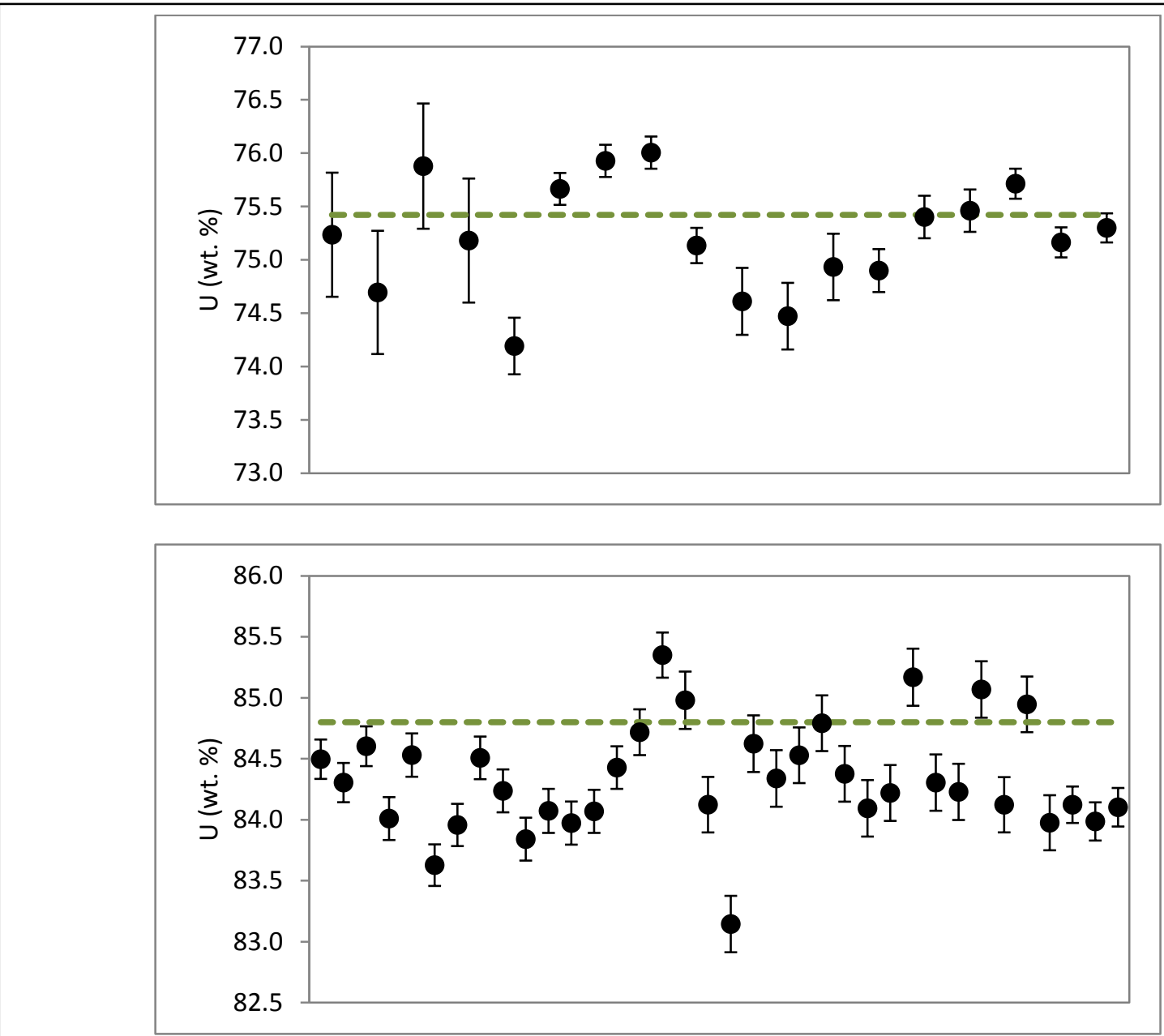

Figure 2. Accuracy and precision of replicate analyses of CUP-2 uranium ore concentrate (75.42 wt. \% U certified value) and CRM 124-1 uranium metal ( 84.8 wt. \% U) measured at Lawrence Livermore National Laboratory over a period of about one year. Uncertainty is calculated following the guidelines of JCGM100:2008.

Standard laboratory safety procedures should be utilized when preparing reagents. All reagents should be prepared in a fume hood. Special caution must be taken when handling and mixing sulfuric acid due to the possibility of vigorous exothermic reaction upon contact with water. Samples with non-natural uranium isotopic compositions, particularly samples with high ${ }^{235} \mathrm{U}$, should be treated as radiological hazards.

\section{Strengths and Weaknesses}

The Davies-Gray technique should be used when uranium concentration data are needed but IDMS measurements are impractical or too costly. Rapid throughput is possible using autotitrations systems (30 or more samples per day). Most auto-titration systems function reliably after initial setup and calibration. Laboratory-grade reagents can be used, and the reagents are 
stocked by most science suppliers. This technique need not be performed in a clean laboratory environment.

The Davies-Gray technique, less precise than IDMS, also lacks the ability to measure trace uranium in samples of interest. Furthermore, Davies-Gray analyses seem to fail at a rate of 5-10 $\%$, usually due to erroneous definition of the potentiometric end-point. Performance of the technique requires a rudimentary understanding of electrochemistry and titration procedures. 


\section{Quadrupole Inductively Coupled Mass Spectrometry}

\section{Introduction}

Quadrupole Inductively Coupled Mass Spectrometry (Q-ICP-MS) is a destructive technique used for elemental and isotopic bulk analysis. Samples are introduced primarily as solutions, however solids can be analyzed as well by coupling with laser ablation or other commercially available solid sourcing systems. Q-ICP-MS provides multi-element analysis for almost all elements in the periodic table aside from the gases. Low detection limits at single parts per trillion and below for most elements and a dynamic range of detection of 9 orders of magnitude or more allows for the analysis of many different types of samples. In addition, rapid sample throughput and relative ease-of-use have made these instruments reliable workhorses. However, this technique is not without its limitations. Matrix interferences from high concentrations of matrix species, especially heavy elements, can reduce overall sensitivity and spectral interferences from molecular polyatomic ions can degrade detection limits. Both types of interferences can be challenging for even the most experienced operator. In addition, physical deposition of the sample can cause contamination/ memory effects in the introduction system. Advances in design that include collision and reaction cells to manage spectral interferences and aerosol dilution to handle heavy matrices have helped to overcome many of these challenges and continuously improve upon this technique.

\section{Instrumentation}

Modern Q-ICP-MS systems offer benchtop platforms and are on average relatively inexpensive $(\sim<250 \mathrm{~K})$. Major manufacturers include Thermo Scientific, Agilent, Perkin Elmer, and Bruker. Most models offer collision cells and some now offer multiple quadrupoles.
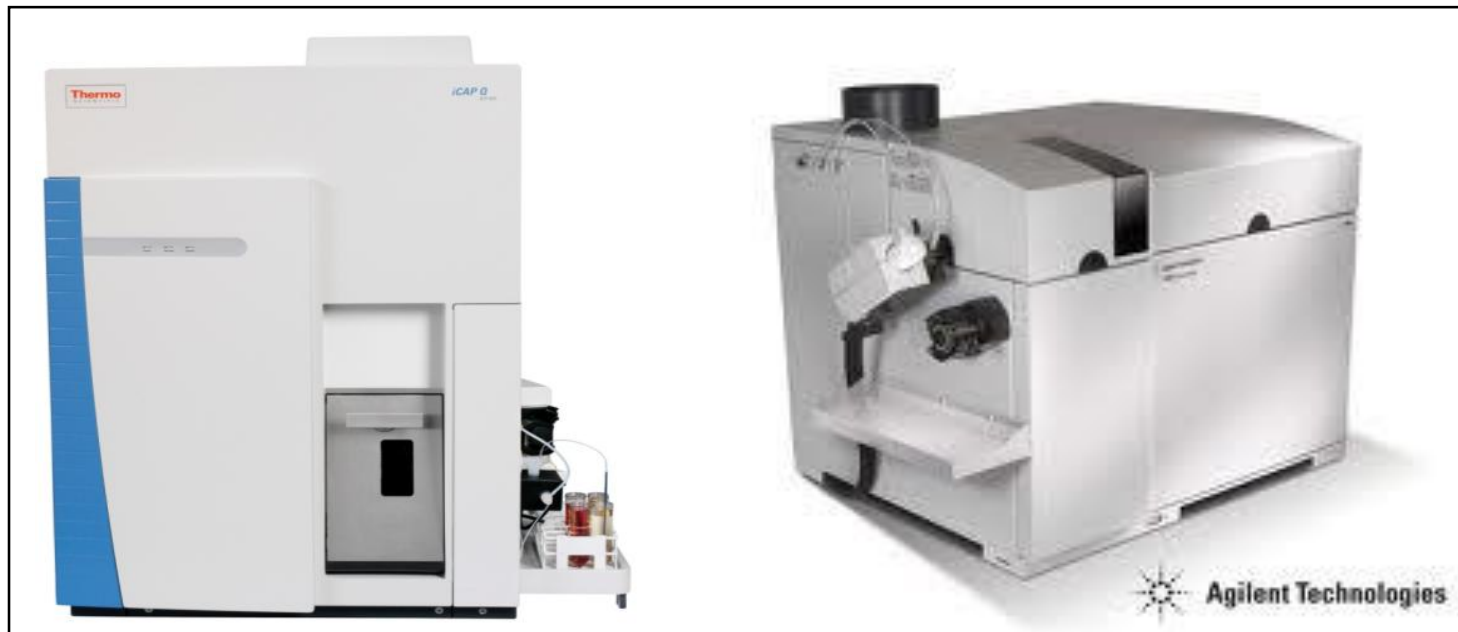

Figure 1. Examples of commercially available Q-ICP-MS. A Thermo Scientific iCAP Q (left inset) and an Agilent 7700 (right inset). 
Typical Q-ICP-MS systems consist of the following components: (1) a sample introduction system used to aerosolize the sample solution, (2) an inductively coupled plasma source that ionizes the aerosol, (3) a vacuum interface that extracts these ions from the plasma source and forms them into a positively charged ion beam, (4) ion optics that focus the ion beam, (5) a quadrupole mass spectrometer that separates the ions in the beam based on mass to charge ratio and (6) a dual mode detector (pulse counting and analog) that measures the intensity of the separated ions. A schematic of a Q-ICP-MS is shown below in Figure 2. These components are discussed separately below.

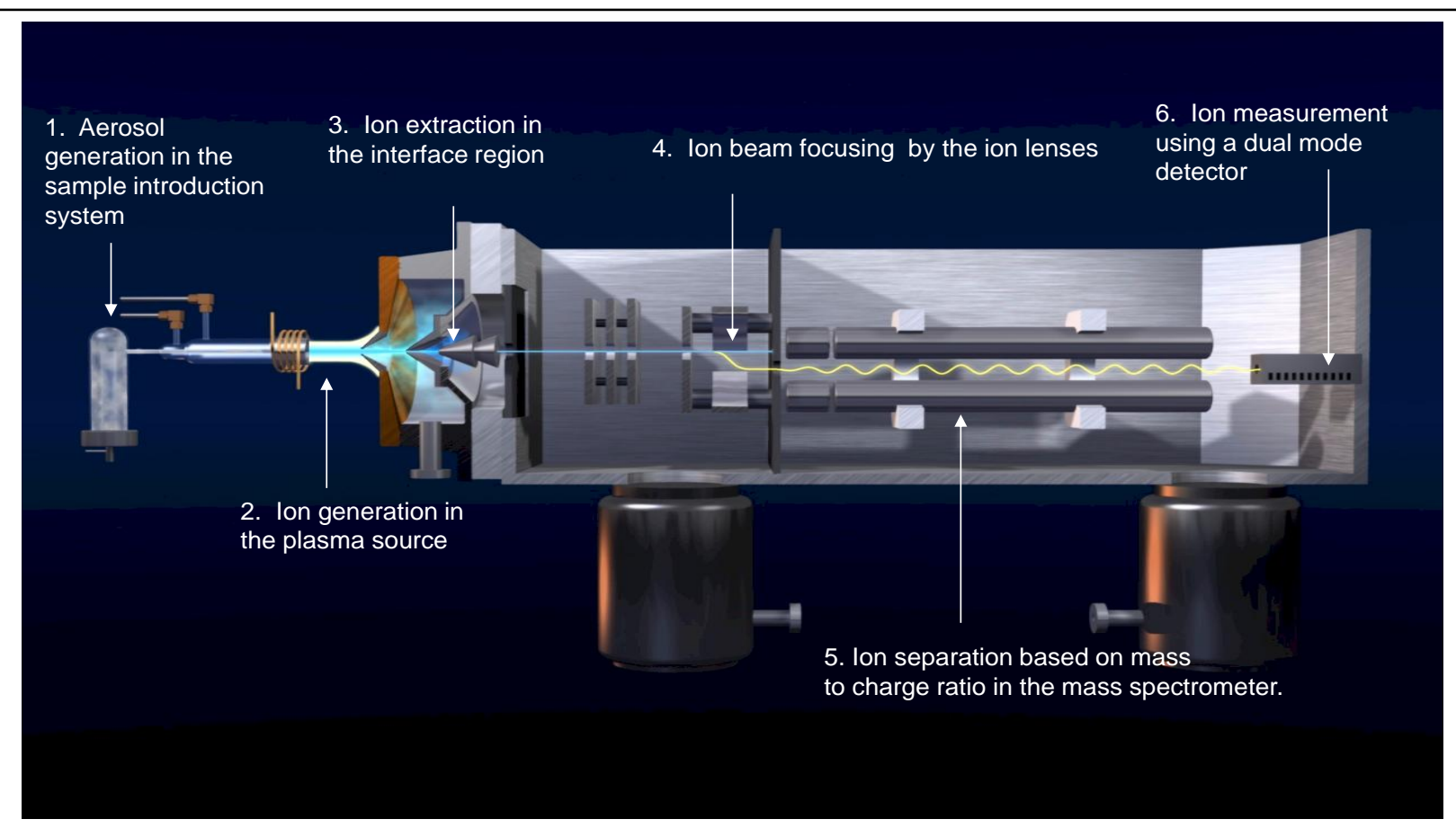

Figure 2. Schematic of Q-ICP-MS

Sample Introduction System. The two primary components of the sample introduction system when introducing solutions are the nebulizer and spray chamber. The sample solution is typically delivered to the nebulizer using a peristaltic pump. The nebulizer is used to break up the liquid into a fine aerosol using argon gas flow. The spray chamber selects only very fine droplets (<10um) from the aerosol to pass into the plasma. This allows for plasma stability and effective ionization. As a consequence of this selection process, only 1-2\% of the original sample is transmitted to the plasma. There is an array of different types of nebulizers and spray chambers to select from to optimize for different sample types such as high dissolved solids or HF resistance.

Inductively Coupled Plasma Source. The plasma source consists of partially ionized argon gas at a temperature of $\sim 8000 \mathrm{~K}$ that is sustained in a quartz torch. The plasma dries, decomposes, atomizes and ionizes the aerosol droplets from the spray chamber. 
Plasma/Vacuum Interface. Ions in the plasma are extracted at the sampling orifice using a set of cones and an extraction lens. The pressure drop from the vacuum system encourages electrons to diffuse out of the beam, creating a positively charged ion beam.

Ion Optics. The extracted ion beam is focused using electrostatic lenses. Neutral species are removed.

Quadrupole Mass Spectrometer. The focused ion beam passes through the quadrupole where rods separate the ions based on their mass to charge ratio.

Dual mode detector. Ions arrive at an ion counter detector called a secondary electron multiplier where they strike a series of dynodes that liberate secondary electrons that cascade and form a pulse that is measured as intensity (counts). The detector has both pulse counting and analog mode which together offer at least 9 orders of magnitude of detection for example from ppt to hundreds of ppm.

Collision-Reaction Cells. Collision cells use Kinetic Energy Discrimination (KED), reducing ion kinetic energy and focusing ions through collisions with a neutral gas of low molecular weight such as He.

\section{Analytical Issues.}

Matrix Interferences. Matrix interferences produce three main effects: suppression of the analyte signal, poor ionization and space charge effects. Suppression of the analyte signal produces an overall loss of sensitivity and can result in poorer precision and accuracy. A large amount of matrix ions can consume the energy of the plasma making it more difficult for analytes with high ionization potentials such as $\mathrm{Zn}$ to ionize in the plasma. Finally, high matrix loading, especially from heavier elements reduce the sensitivity of lighter elements due to space charge effects. The increased density of ions causes them to react and repel each other. Since the lighter ions have less kinetic energy and momentum they are more easily deflected out of the ion beam during these reactions, while the heavy ions "push through". For example, an element such as lithium will suffer large transmission losses in a uranium matrix. Matrix interferences can be managed by optimizing operating parameters to maximize ionization of the plasma and optimize extraction/transmission of the lighter elements. Matrix matching calibration standards to unknowns and employing internal standards to normalize matrix effects are key to successful analyses. When matrix matching is not an option the more time consuming standard addition and isotope dilution techniques may be needed, or the matrix may need to be removed all together. 
Spectral Interferences. Spectral interferences comprise of both isobaric interferences and molecular polyatomic interferences. Isobaric interferences occur when one elemental isotope shares the same mass with another isotope of a different element. An example is ${ }^{114} \mathrm{Sn}$ on ${ }^{114} \mathrm{Cd}$. Molecular polyatomic interferences occur when a molecular ion interferes with the isotope of a desired element such as ${ }^{56} \mathrm{ArO}$ on ${ }^{56} \mathrm{Fe}$. Oxides, hydroxides and argides are the main interferences and are most severe in mass/charge values

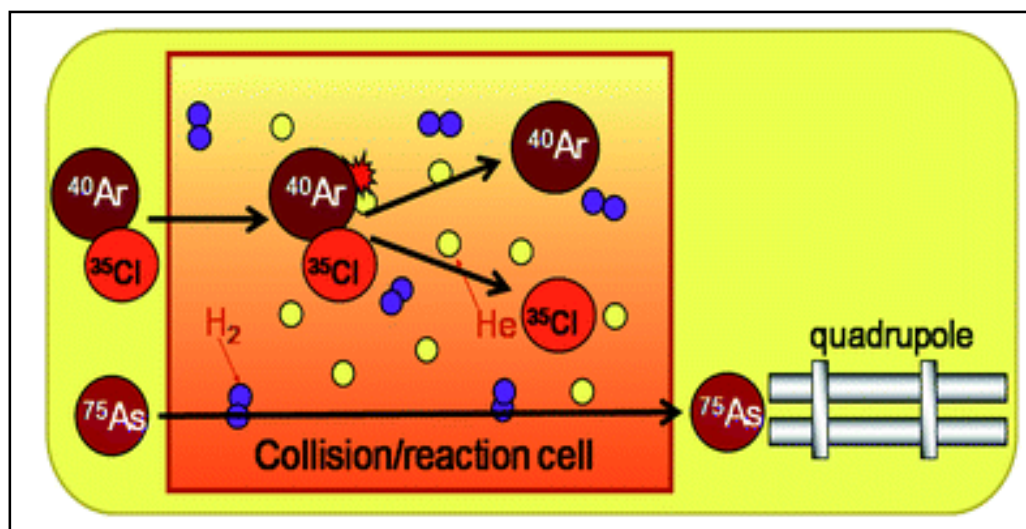

Figure 3. Removal of polyatomic interference $\mathrm{ArCl}$ from ${ }^{75} \mathrm{As}$ using a collision/reaction cell.

less than 82. Many of the isobaric interferences can be corrected using straightforward mathematical interference corrections. Correcting for molecular polyatomic interferences is more complicated and can produce a large amount of uncertainty in the end result. Spectral interferences resulting from the matrix such as the doubly charged ${ }^{238} \mathrm{U}^{++}(\mathrm{m} / \mathrm{z}=238 / 2)$ on ${ }^{119} \mathrm{Sn}$ require matrix matching of unknowns and standards. Another option in managing interferences is to reduce the interference as much as possible using collision/reaction cells. The isobaric interference of ${ }^{40} \mathrm{Ar}$ on ${ }^{40} \mathrm{Ca}$ and the molecular polyatomic interference of ${ }^{75} \mathrm{ArCl}$ on ${ }^{75} \mathrm{As}$ are efficiently removed in these cells. Reaction cells are especially effective in removing background interferences of low masses. Limitations of reaction cells include transmission loss from scattering due to the reaction or collision gas and the possibility of reaction products forming new spectral interferences.

Memory. Deposition in the sample interface can cause elevated blank levels and drift. Combined with the matrix space charge effects described above, samples often must be diluted and as a result detection limits are increased. Adequate rinseout between samples can alleviate this issue. In addition, challenging matrices can be assigned their own introduction sets when cost is not an issue.

Sample preparation. Sample preparation, in general, is simple and quick when solutions can be run straight or only require minimal dilution. When analyte concentrations approach detection limits, additional effort is often required. A very clean sample with little matrix may benefit from pre-concentration to enhance detection limits. Difficult matrices may require standard addition, isotope dilution or matrix separation. In the case of standard addition, sample preparation becomes much more laborious as it requires at least 3 preparations per sample. Isotope analysis may require isotope dilution and chemical purification to achieve good precision. Sample dissolution of solid samples must be carefully planned and controlled to 
achieve full dissolution and sample stability while avoiding contamination from the dissolution procedure. Luckily there is an abundance of resources available on-line and in the literature to assist in the development of successful sample preparation procedures.

\section{Application}

As seen in Table 1, Q-ICP-MS can be used throughout most of the fuel cycle to identify signatures. Beginning with ore materials and following through to fuel fabrication materials such as $\mathrm{UO}_{2}$ and $\mathrm{U}_{3} \mathrm{O}_{8}$. The challenges for analyzing these materials are completely different at each end of the spectrum. Ore materials contain plenty of impurities for easy detection, however they can be challenging to dissolve and keep stable in solution. Conversely, the pure fuel products are easy to put into solution, but many of the analytes are at or near detection limits. For example, in order to follow rare earth signatures through from the ore material, it may be necessary to employ matrix separation of the fuel product. When analyzing concentrated fuel products or reprocessing material, one must consider radiological controls. Unless systems are installed in a "hot" enclosure, radiological limits may dictate analytical technique. If the Q-ICPMS is used for other applications, components of the introduction system should be carefully removed and isolated after analysis to prevent cross-contamination.

\section{Strengths and weakness of the Q-ICP-MS technique}

Strengths. Q-ICP-MS offers enormous flexibility in analyzing a wide array of sample types. It offers low detection limits, great accuracy and high precision. The ability to perform multi-element and isotopic analysis coupled with rapid analysis time, ease-of-use and low cost make Q-ICP-MS a very powerful technique.

Weakness. While Q-ICP-MS is a relatively inexpensive workhorse, it cannot compete with the interference resolution capabilities that the high resolution mass spectrometers (HRICP-MS) offer. In terms of isotopic ratio work it is not as sensitive nor as precise as high resolution and multi-collector ICP-MS or TIMS. 


\section{X-ray fluorescence spectrometry}

\section{Introduction}

$\mathrm{X}$-ray fluorescence (XRF) spectrometry is a non-destructive analytical technique used to determine the chemical composition of solid and liquid samples. Samples can be analyzed as bulk solids (e.g. metals, rocks, ceramics, etc.), pressed pellets, loose powders, or liquids. Although XRF spectrometry lacks the low detection limits and high precision of ICPMS, modern $\mathrm{XRF}$ instruments are capable of accurately measuring the concentration of most elements (typically $\mathrm{Na}$ to $\mathrm{U}$ ) in a matter of minutes with little to no sample preparation. XRF spectrometry is particularly useful in cases where sample alteration must be kept to a minimum, and can be used as a screening tool to guide future analytical work. XRF spectrometers are often used in tandem with microbeam technologies such as scanning electron microscopy (SEM), providing these instruments with an elemental quantification capability. In addition, most XRF instruments can be customized for specific applications, such as the measurement of elements lighter than sodium.

\section{Basic Theory}

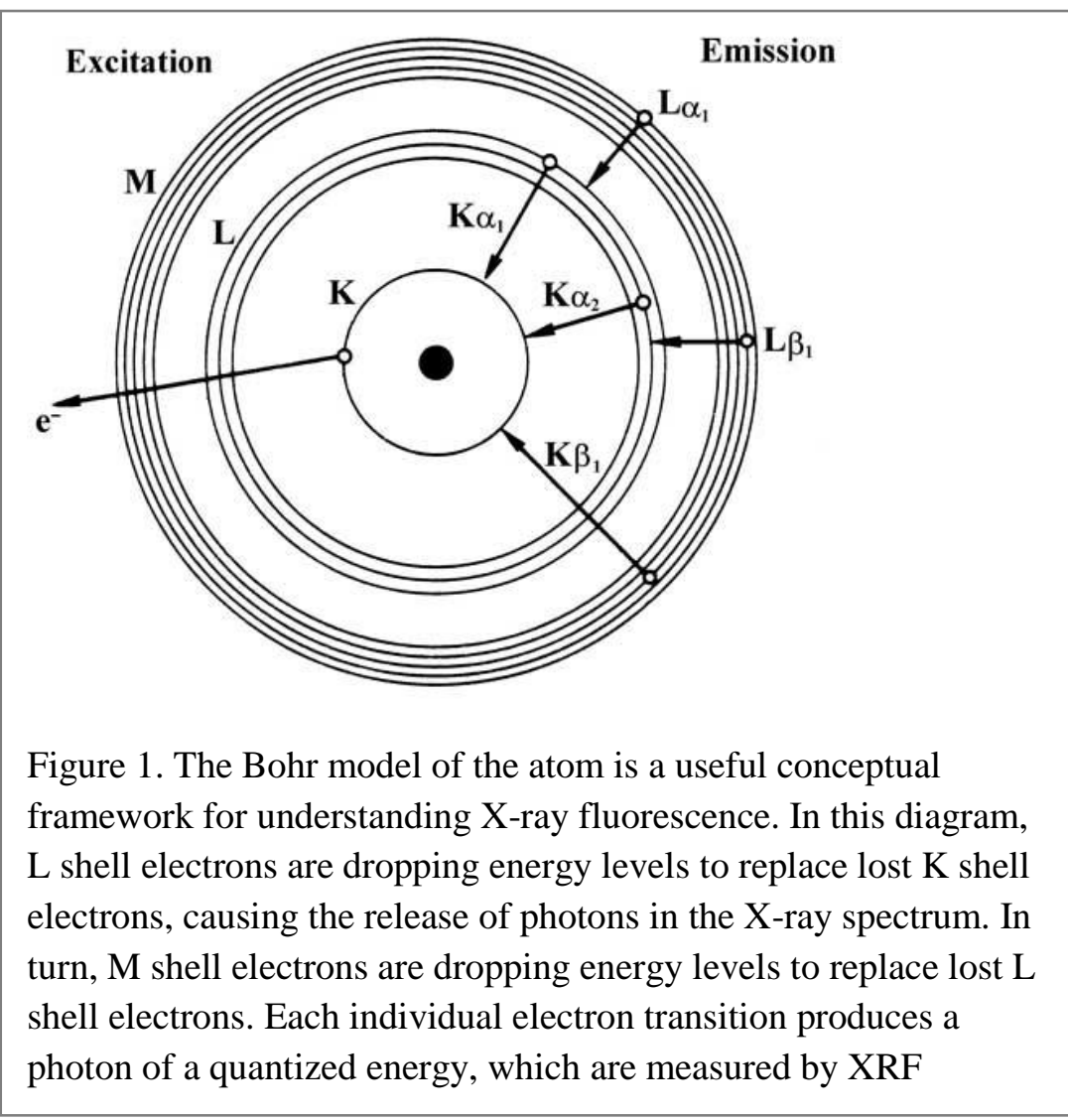

Atoms produce electromagnetic radiation in the X-ray spectrum $(0.01$ to $10 \mathrm{~nm})$ when inner-shell electrons are lost and outershell electrons drop energy levels to replace them in a process called fluorescence (Figure 1). As the electron configurations of a particular element are defined by the quantum numbers of each electron orbital, the $\mathrm{X}$-rays produced by electron transitions have energies characteristic to each element in the sample. XRF spectrometry takes advantage of this behavior by measuring the characteristic $\mathrm{X}$-rays produced by each element. As 
the number of characteristic X-rays produced by a sample is directly proportional to the concentration of each element in the sample, the chemical composition of the sample can be determined.

A schematic diagram of a WD-XRF is presented in Figure 2. A primary X-ray beam, generated by an X-ray tube with a $\mathrm{Rh}$, Mo, or $\mathrm{W}$ anode target, is used to trigger inner-shell electron loss in a sample. As outer-shell electrons in the sample drop energy levels to replace the lost inner-shell electrons, characteristic X-rays are released (Figure 3). The beam of characteristic X-rays is collimated and directed towards a detector (energy-dispersive XRF, or ED-XRF) or a crystal (wavelength-dispersive XRF, or WD-XRF). In ED-XRF, a silicon detector is used to discriminate between the different energies of the photons in the characteristic X-ray beam, and each incoming photon is binned by energy and counted. In WD-XRF, the characteristic X-ray beam is directed at an angle to a crystal with a given d-spacing. As only photons with the correct energy to solve for Bragg's equation at a given angle will remain in phase and be diffracted, all other X-rays will be obliterated upon interaction with the crystal. The in-phase X-rays are directed towards a detector and counted. In this way, each X-ray energy of interest can be selected for by simply changing the angle at which the characteristic X-ray beam reaches the crystal. Multiple crystals, with different d-spacing, can be used in sequence to measure all elements of interest.

Elemental concentrations are quantified relative to a calibration curve for each element. Ideally, a matrix-matched calibration curve is used, constructed for the elements of interest using a series of standards prepared similarly to the sample. In cases where a matrix-matched calibration curve cannot be obtained or is impractical, modern XRF data reduction software can be used to calculate concentration results semiquantitatively on the basis of "fundamental parameters". Assumptions must be made about sample geometry, homogeneity, and thickness to produce accurate results. In most cases, the "fundamental parameters" approach is capable of accurately determining the chemical composition of a sample to within $10 \%$ precision.

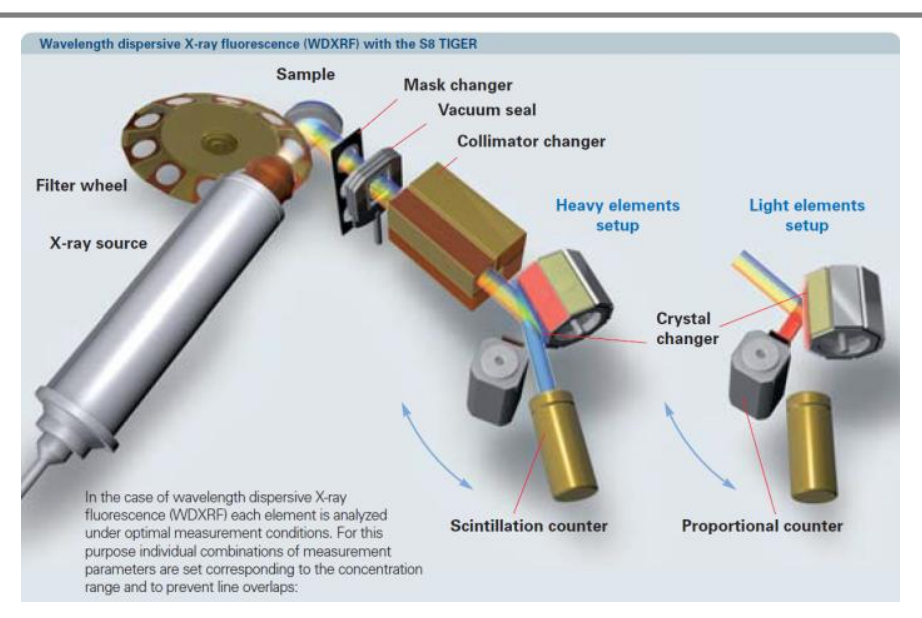

Figure 2. A schematic diagram of the X-ray beam path of the Bruker S8 Tiger WD-XRF spectrometer. In this diagram, the $\mathrm{X}$-ray tube is generating a primary X-ray beam, causing inner-shell electron loss in a sample. The beam of characteristic X-rays produced by the sample is guided to a crystal set at an angle. Out-of-phase X-rays are obliterated, while in-phase X-rays are diffracted off of the crystal and directed towards one of two detectors. 


\section{Instrumentation}

Top-of-the-line WD-XRF spectrometers designed for bulk sample measurement, manufactured by Bruker, PANalytical, Rigaku, and other companies, can cost up to $\$ 250 \mathrm{k}$. Lower-cost XRF spectrometers typically utilize a less-powerful X-ray tube and lack customizable options, limiting the scope of applications the instrument can be used for. For bulk sample analysis, WD-XRF instruments are more versatile than ED-XRF instruments. ED-XRF spectrometers are frequently installed on SEMs, but some high-end SEMs utilize WD-XRF spectrometers.

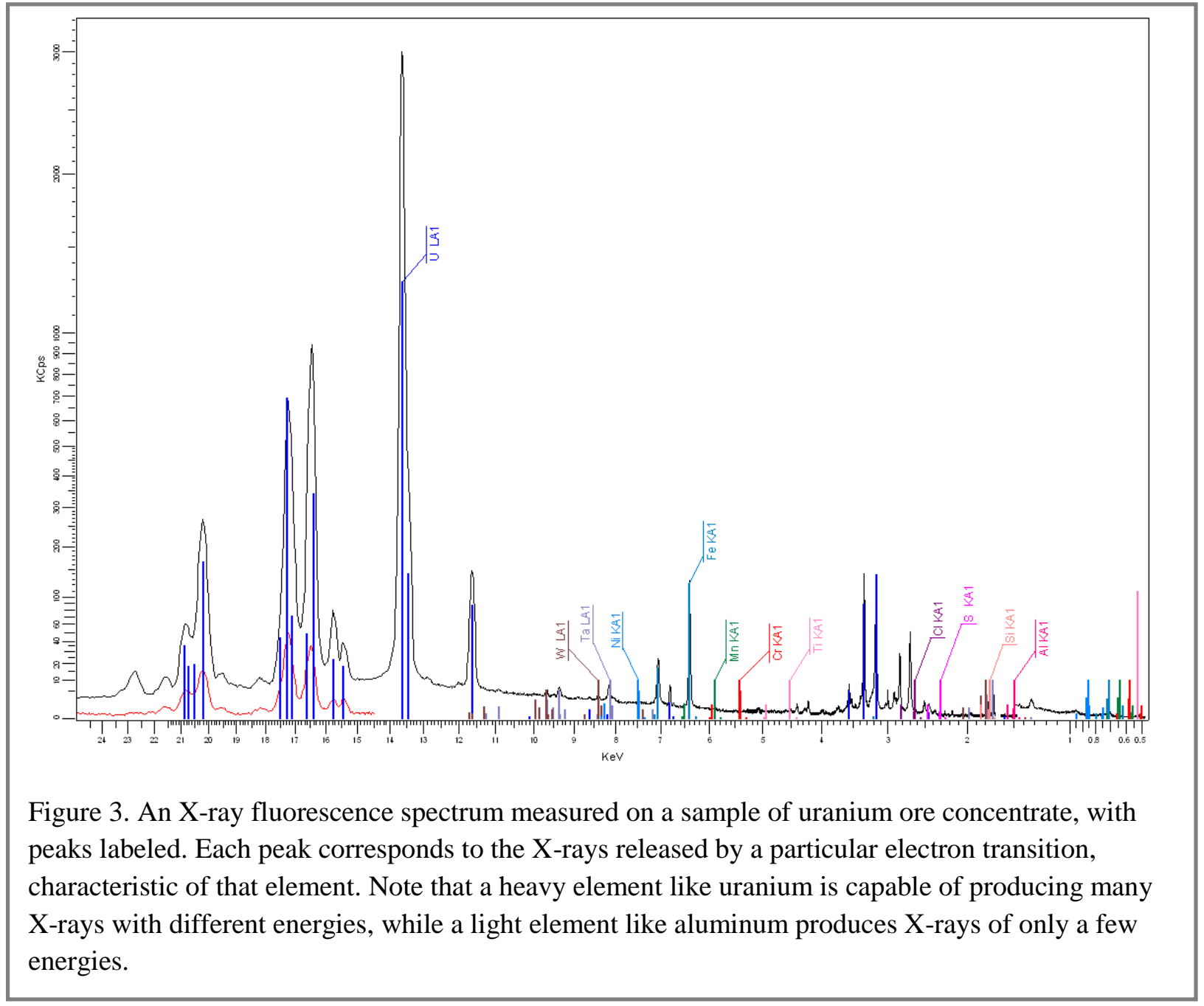

For most geological and environmental applications, an X-ray tube with a $\mathrm{Rh}$ target anode is preferred. The characteristic $\mathrm{Rh} \mathrm{X}$-rays generated by the $\mathrm{X}$-ray tube are of the requisite energy to trigger inner-shell electron loss in light and heavy elements. However, for certain industrial applications, a Mo or $\mathrm{W}$ anode target is preferred. The voltage and current of the X-ray tube can be adjusted to optimize the measurement for a particular set of analytes. Primary beam 
filters can be applied to remove line overlaps caused by the characteristic $\mathrm{Rh} \mathrm{X}$-rays generated by the X-ray tube. Modern X-ray tubes have a lifespan of several years if maintained properly. Care should be taken to prevent sample material from accumulating on the X-ray tube window (usually a thin, fragile piece of Be). Sample spills should be avoided at all costs, and any loose sample material within the spectrometer should be cleaned up immediately. In order to minimize air molecule interference with the characteristic X-rays generated by the sample, excitation of the sample is usually performed in a vacuum environment. For liquid and loose powder samples, a helium atmosphere can be used instead.

Although the X-ray tube (and, to a lesser extent, the characteristic X-rays produced by the sample) represents an acute radiological hazard, modern XRF instruments are constructed with redundant interlocks and materials opaque to X-rays, allowing for safe working conditions at all times. Care must be taken not to override the interlocks or alter the X-ray safety systems of the instrument. Special care must be taken if radioactive or hazardous materials are to be analyzed by XRF.

\section{Analytical Issues}

Most samples smaller than the XRF sample holder size can be analyzed with no sample preparation. Ideally, a flat and polished face should be analyzed for best results. Samples must be large enough to completely cover the analytical area of the sample holder. For smaller samples, or for measuring a small portion of a larger sample, masks of different sizes can be installed to isolate the portion of interest. It is important to note that bulk sample XRF spectrometers are not capable of assessing micro- and meso-scale sample heterogeneity. XRF chemical composition results that are discrepant with ICPMS results may be due to sample heterogeneity not captured by either analytical technique.

Loose powders can be analyzed on many modern XRF instruments. Powders are loaded into sample cups with a thin film (usually polypropylene) used to hold the powder in place. As the thin film is transparent to $\mathrm{X}$-rays, the powder sample can be analyzed directly with minimal interference from the film. Care must be taken not to damage the film, which can result in film breakage and instrument contamination. Powder samples should never be run in a vacuum environment. Many modern XRF spectrometers utilize a helium atmospheric mode to equalize pressure in the sample chamber while providing minimal atmospheric interference with X-rays produced by the sample. Loose powder analyses are ideal in cases where sample mass is limited and sample aliquots are to be used for additional analytical work.

Samples are commonly converted to glass discs and pressed pellets for XRF analysis. Although the non-destructive nature of the technique is lost, it is easier to build a matrix-matched calibration curve using these sample matrices. Glass discs can be manufactured by melting and 
rapidly cooling the sample in a platinum crucible. Commercial fluxer instruments are ideal for making glass discs, but laboratory furnaces can also be used. Pressed pellets can be made using a standard pellet press. Corrections must be performed to account for the chemical composition of any flux or binder used in sample preparation. Loss on ignition should be calculated for samples converted to glass discs.

In cases where sample mass is limited, sample thickness is a concern. In most cases, quantification of sample composition is performed under the assumption that, for a given X-ray tube voltage and current setting, the maximum number of X-rays a sample can theoretically generate are actually produced ("infinite thickness"). In cases where only a thin layer of sample can be used (10s to $100 \mathrm{~s}$ of $\mu \mathrm{m}$ ), the quantitative results calculations will systematically underestimate heavy element concentrations, generating inaccurate results. If thin samples must be analyzed, a calibration curve should be built by analyzing a series of standards of the same thickness as the samples of interest.

\section{Strengths and Weaknesses}

XRF spectrometry is a well-understood and widely-used analytical technique in many industrial and research settings, and is particularly useful in applications where chemical composition data are required but high-precision ICPMS data are unnecessary (e.g. industrial quality control). $\mathrm{XRF}$ analyses are rapid and non-destructive, allowing for rapid turnaround time and re-allocation of sample for additional analytical work. XRF instruments are less expensive, more userfriendly, and more versatile than ICPMS instruments, and do not require a wet laboratory setup. Most XRF instruments can be operated by technicians with little laboratory experience. XRF spectrometry is useful for measuring analytes that ionize poorly in ICPMS (e.g. Br, S, P).

However, XRF results are almost always less precise, and sometimes less accurate, than ICPMS results. XRF measurement detection limits are on the order of parts per million, limiting the utility of this technique for the analysis of trace constituents. Elements lighter than sodium are difficult to analyze with most XRF instruments. Calibration standards do not exist for many sample matrices of interest. Interferences can be difficult or impossible to resolve in some cases (e.g. U interference on Mo and Zr). 


\section{Thermal Ionization Mass Spectrometry}

\section{Introduction}

Thermal ionization mass spectrometry (TIMS) is used to measure isotope ratios of bulk samples and particles. It has been the traditional mainstay of isotopic ratio measurement for nuclear forensics applications, but has been augmented, and in some cases replaced, by multicollector inductively coupled mass spectrometry (MC-ICPMS) over the last 10 years. Thermal ionization mass spectrometry is the preferred technique for elements that have low ionization potentials (i.e. require low amounts of energy to form ions by removing electrons from gaseous atoms). Typical elements analyzed for isotopic composition (IC) by TIMS include strontium, lead, the lathanides, and the actinides. Concentration determinations can also be completed using artificially enriched isotopic tracers by a method known as isotope dilution (ID).

\section{Instrumentation}

Most TIMS used for forensic analysis are obtained from commercial vendors at a cost of $\sim$ \$0.75-1M US dollars (Figure 1). These instruments have very similar designs and roughly equivalent
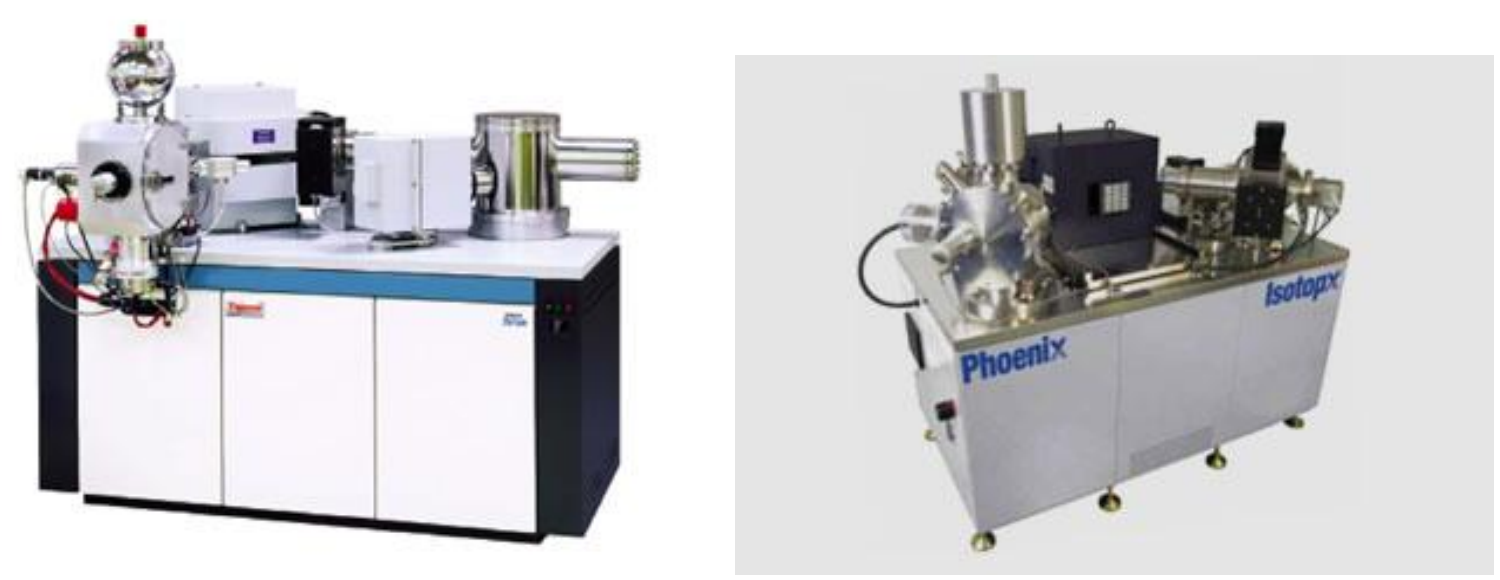

Figure 1. Commercially available thermal ionization mass spectrometers. A ThermoFisher Triton (left inset) and an Isotopx Phoenix (right inset).

capabilities. Thermal ionization mass spectrometers have three main components: (1) an ion source that is used to produce ions and accelerate them into the instrument, (2) a magnetic sector that produces a magnetic field that bends the ion beam and separates ions of different masses into individual beams, and (3) a collector array used to measure the intensity of the ion beams produced by isotopes of specific masses. An illustration of a typical TIMS is presented in Figure 2. These components are discussed separately below. 


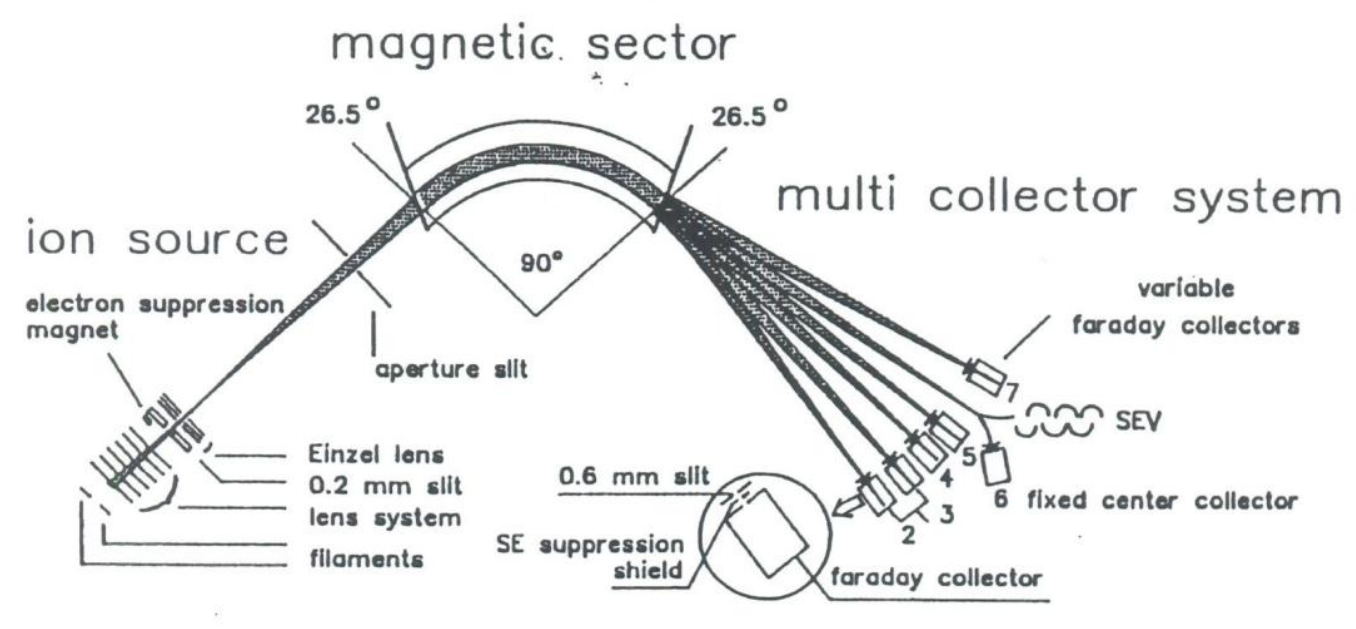

Figure 2. Schematic of TIMS instrument.

Ion Source. The ion source of a TIMS is composed of a wire filament made of Re, Ta, or W. The sample is loaded onto the filament in a very small drop of weak acid and dried down on a bench top by applying a small current. In some cases the sample is loaded with emitters, such as carbon or $\mathrm{Ta}_{2} \mathrm{O}_{5}$, that enhance the ionization efficiency of a particular element or help to convert it to a molecular species, such as $\mathrm{NdO}^{+}$, that ionizes more readily than the elemental species of the element $\left(\mathrm{Nd}^{+}\right)$. In a modern TIMS the filaments are loaded onto a turret that contains up to 20 or so samples. The turret is placed in the instrument under vacuum where a current of 1000 to $3000 \mathrm{~mA}$ is passed through the filament. This current both evaporates the loaded element, forming a gas, and ionizes it by stripping an electron. In some cases, ionization efficiency is improved by adding a second or third filament. These filaments are known as ionization filaments and typically run at $4500 \mathrm{~mA}$, whereas the filaments on which the samples are loaded are known as evaporation filaments. The number of ions measured in the detectors relative to number of atoms loaded is defined as the ionization efficiency and varies from approximately $0.1 \%$ for $\mathrm{U}$ to $4 \%$ for $\mathrm{NdO}+$. The ions produced on the filament(s) in the source are accelerated through a series of ion lenses towards the magnetic sector with $\sim 10 \mathrm{kV}$ accelerating potential. The direction of the beam is guided by a series of ion lenses called a lens stack.

Magnetic sector. The magnetic sector is sometimes call a mass analyzer and produces a magnetic field that is used to bend the ion beam $90^{\circ}$ towards the collector array. The extent to which the ion beam bends in the magnetic field depends on the velocity of the individual ions, the strength of the magnetic field, and the mass of the ions. Because the accelerating potential and magnetic fields are held constant, the extent to which an individual ion bends in the magnetic sector depends only on its mass. Thus, ions exiting the magnetic sector are divided 
into ion beams characterized by specific masses. The magnetic field is set to insure that the mass of interest is centered in the middle of the collector array.

Collector arrays. Collect arrays of modern commercially available mass spectrometers are composed of a combination of Faraday cups, a central ion counter (secondary electron multiplier or ion counting Daly detector), and miniaturized electron multipliers. The axial of central detector is fixed and does not move, whereas the detectors on either side of the axial detector are movable so that they can be placed in the proper location to measure ion beams associated with a variety of elemental and molecular species. As a consequence a single mass spectrometer has the capability to measure multiple masses for virtually all elements on the periodic table. Large ion beams are measured using Faraday cups. These detectors provide very reproducible measurements and are used to provide the most accurate isotopic measurements. However, they require relatively large ion beams and are consequently only used for elements that ionize efficiently (e.g. $\mathrm{Sr}, \mathrm{Nd}$, or $\mathrm{Pb}$ ) or are present in very large abundances (e.g. $\mathrm{U}$ in uranium ore concentrate, $\mathrm{UF}_{\mathrm{x}}$, or $\mathrm{U}$ metal). Ion counters are used to measure beams with significantly lower intensities. Whereas a Faraday cup detector has trouble with signals less than $1 \times 10^{-13} \mathrm{~A}$, and ion counter can comfortably measure signals as low a $1 \times 10^{-15} \mathrm{~A}$. The short coming of the ion counter measurements, however, is that they are not as accurate or precise as Faraday cup measurements. Nevertheless, ion counters are used extensively in nuclear forensics because they provide the only means available to measure small beams and because isotopic variations in many samples of interest are large compared to the uncertainties associated with these measurements.

\section{Analytical Issues}

The isotopic ratio determinations require corrections for mass fractionation associated with evaporation of the sample from the filament, as well as occasionally for interferences associated with isobars of elements with similar masses to those of interest. In addition, significant sample preparation is required prior to loading of the filament.

Instrument mass fractionation. Mass fractionation occurs as a result preferentially evaporating light elements from the filament during heating (Figure 3). This fractionation is mass dependant and can be corrected for in three ways. In the first, an elemental ratio of a known value is used to apply a correction to isotopic ratios of interest. For example, when measuring ${ }^{143} \mathrm{Nd} /{ }^{144} \mathrm{Nd}$ the ${ }^{146} \mathrm{Nd} /{ }^{144} \mathrm{Nd}$ also measured and assumed to be 0.7219 . Deviations of the measured ${ }^{146} \mathrm{Nd} /{ }^{144} \mathrm{Nd}$ are applied to ${ }^{143} \mathrm{Nd} /{ }^{144} \mathrm{Nd}$ taking into account that the difference in mass between ${ }^{146} \mathrm{Nd}$ and ${ }^{144} \mathrm{Nd}$ is roughly twice that for ${ }^{143} \mathrm{Nd}$ and ${ }^{144} \mathrm{Nd}$. In many cases, there are not enough stable isotopes of an element to apply this type of internal fractionation correct. In natural systems, such as common $\mathrm{U}$ or $\mathrm{Pb}$, artificially produced tracers with known isotope ratios can be added and used to correct for fractionation. Thus, tracers with known ${ }^{233} \mathrm{U} /{ }^{236} \mathrm{U}$ and ${ }^{205} \mathrm{~Pb} /{ }^{202} \mathrm{~Pb}$ can be added to natural $\mathrm{U}$ or $\mathrm{Pb}$ to correct for fractionation, providing the most accurate ratio determinations. In cases where no artificially produced tracer is available, 
fractionation can be corrected using a second technique. In this case the magnitude of the average mass fraction occurring in the instrument is determined by running standards and comparing the values of measured ratios to certified or accepted values. Unknowns are corrected for mass fractionation using this fractionation correction. It should be noted that this correction is significantly less precise than internal fractionation corrections because fraction of mass spectrometry runs are never identical. The final technique that is used in called "Total evaporation". In this method, the filament is heated throughout the run as isotope ratios are determined. No fractionation correction is applied because the sample is run to exhaustion and the final ratio is determined from the sum of intensities of each mass collected throughout the run.

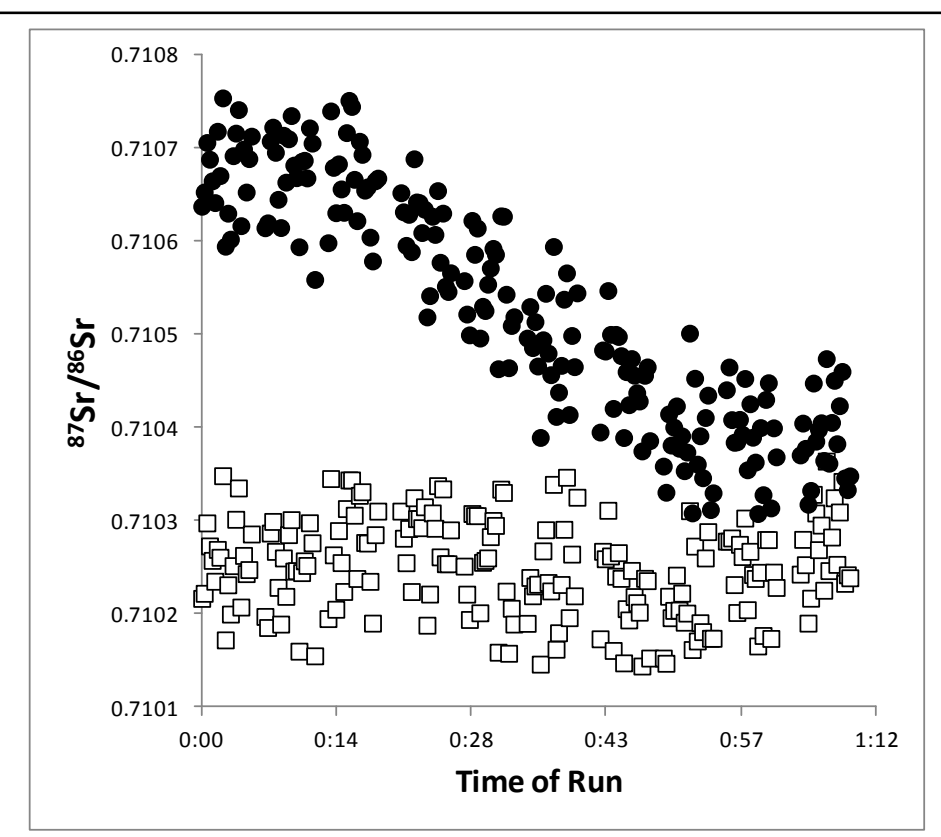

Figure 3. Isotopic variation of $\mathrm{Sr}$ isotopes due to instrumental mass fractionation associated with evaporation. Filled circles are uncorrected for mass fractionation, whereas open squares are corrected assuming ${ }^{86} \mathrm{Sr} /{ }^{88} \mathrm{Sr}=0.1194$.

\section{Interfering element corrections.} Interfering element corrections are necessary when chemical separations are not complete and multiple elements with essentially the same mass are loaded onto the filament. Examples of isobar pairs that need to be accounted for are ${ }^{87} \mathrm{Rb}-{ }^{87} \mathrm{Sr},{ }^{144} \mathrm{Sm}^{-144} \mathrm{Nd},{ }^{142} \mathrm{Nd}^{18} \mathrm{O}-$ ${ }^{144} \mathrm{Nd}^{16} \mathrm{O},{ }^{238} \mathrm{U}_{-}{ }^{239} \mathrm{Pu}$, and ${ }^{241} \mathrm{Pu}-$ ${ }^{241} \mathrm{Am}$. If a trace amount of an interfering element is present on the filament it can be subtracted using the measured value of an uninterfered isotope of the interfering element, such as ${ }^{85} \mathrm{Rb}$, by assuming a constant ratio of this isotope to the interfering isotope (i.e. ${ }^{85} \mathrm{Rb} /{ }^{87} \mathrm{Rb}=2.603$ ). $\quad$ Large interfering element corrections, however, will compromise the

analysis because of the inability to account for instrument mass fractionation of the interfering element. Likewise, measurements are compromised by interferences from elements with poorly known isotopic compositions.

Sample preparation. The TIMS technique does not have high mass resolution so that signals from isotopes of different elements cannot be distinguished. Thus, samples must be chemically purified prior to analysis. This usually involves ion chromatography that requires a wet chemistry laboratory. For elements present in airborne particulates, such as $\mathrm{Sr}, \mathrm{Nd}$, and $\mathrm{Pb}$, a clean laboratory is needed. Samples are loaded in a diluted salt matrix (weak hydrochloric or 
nitric acids) or on resin beads. The amount of sample needed is highly variable and depends on the ionization potential of the element, the detector type used for the analysis, and the details of the analytical method needed to make all relevant isotope ratio measurements given the detector configuration of the TIMS used for the analysis. In general, the amount of sample needed ranges from picrogram to microgram quantities.

\section{Strengths and weakness of the TIMS technique}

Strengths. Thermal ionization mass spectrometry is very precise as a result of the small amount of instrument mass fractionation (typically $\sim 0.1 \%$ per atomic mass unit) compared to other techniques. For $\mathrm{Sr}$ and $\mathrm{Nd}$ precisions as low as $0.0015 \%$ (two standard deviation of replicate analyses on standards) are achievable. Typical uncertainty on externally fractionation corrected $\mathrm{U}$ is $<0.05 \%$, and as low as $0.01 \% \%$ if a ${ }^{233} \mathrm{U} /{ }^{236} \mathrm{U}$ is used. In addition, interferences from molecular species are for the most part trivial. It is idea for high precision isotope ratio determinations for elements such as $\mathrm{Sr}, \mathrm{Nd}$, and $\mathrm{Pb}$. High vacuum throughout the instrument combined with ion beams produced by thermal ionization result in high abundance sensitivity for the TIMS. Abundance sensitivity is essentially an estimation of how much signal from an adjacent ion beam strays into a detector. In normal operating modes abundance sensitivities for commercially available TIMS are $\sim 2 \times 10^{-6}$ or 2 ions per million and can reach as low as $2 \times 10^{-9}$ when energy filters are applied. Thus, isotopic measurements of isotopes that are present in very low abundances are well suited for the TIMS.

Weakness. Thermal ionization has several weaknesses as well. The first is that analyses take a relatively long time and often require significant operator input. A second weakness is that ionization efficiency for various elements are low, so that large amounts of sample are sometimes required to obtain enough signal to acquire isotopic ration with sufficiently low analytical uncertainties. In some cases, elements ionize so poorly that they cannot be analyzed by TIMS. 


\section{Multi-Collector Inductively-Coupled Plasma Mass Spectrometry}

\section{Introduction}

Multi-collector inductively-coupled plasma mass spectrometers (MC-ICP-MS) are used to measure isotope ratios of bulk samples at several stages in the fuel cycle. These instruments represent relatively new technology; the first commercially available MC-ICP-MS instruments were produced in the mid-1990's. The application of MC-ICP mass spectrometry is similar to that of TIMS. The primary difference between the two instruments is that with MC-ICP-MS samples are ionized using a plasma, and therefore this method is preferred for analysis of elements with low ionization potentials that would otherwise be difficult to analyze by TIMS. However, a plasma source creates an intrinsically unstable ion beam. By coupling the ICP source with a magnetic sector and an array of multiple collectors, it is possible to simultaneously measure ion beam intensities for all desired masses, thereby minimizing analytical noise generated by the plasma source. MC-ICP mass spectrometry is used to measure isotope ratios of many major and trace elements in fuel cycle materials, including $\mathrm{U}$ and the actinides, $\mathrm{Pb}, \mathrm{Sr}$ and the lanthanides.

\section{Instrumentation}

At present, there are two commercially available MC-ICP-MS instruments: the Plasma II, by Nu Instruments Ltd., and the NEPTUNE Plus, by Thermo Scientific (Figure 1). Both instruments are appropriate for use in measuring isotopic signatures in fuel cycle materials.
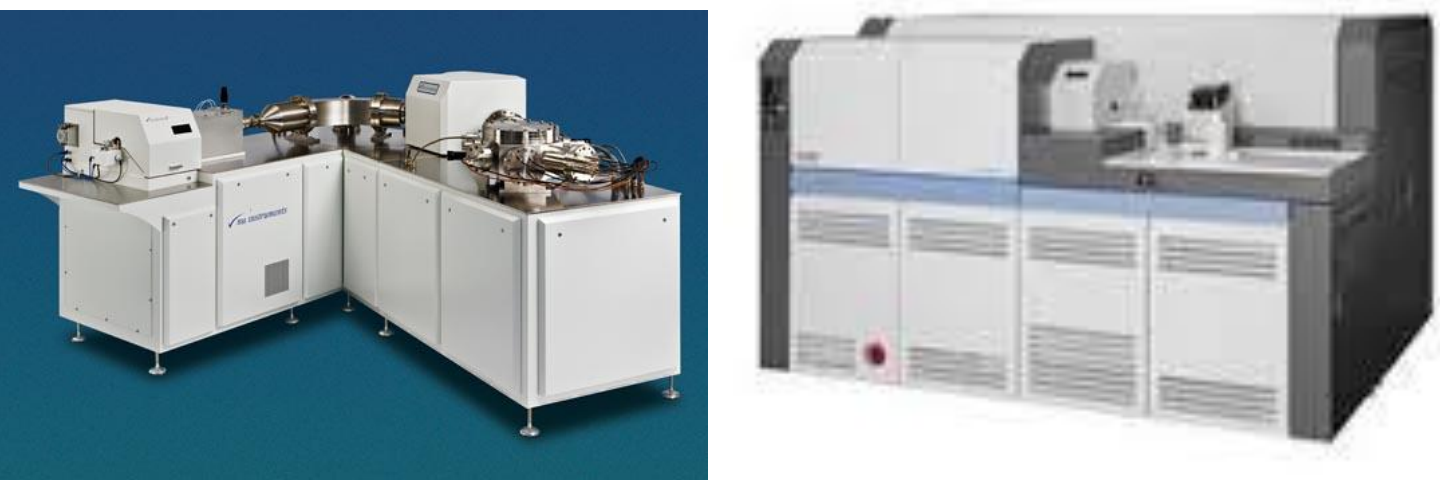

Figure 1. Commercially available MC-ICP-MS instruments. Nu Plasma II (left) and Thermo Scientific NEPTUNE Plus (right).

The most common analyte for MC-ICP-MS analysis consists of the sample dissolved in weak acid, typically $\mathrm{HNO}_{3}$. In most instrument configurations, the sample is passed through a desolvating nebulizer to remove the solvent from the sample, and then it is introduced into the base of an inductively coupled plasma. Argon is used as the plasma gas. As the sample flows 
into and through the plasma, it is desolvated (if a desolvating nebulizer is not used a separate component of the sample introduction system), vaporized, atomized and finally ionized. Ionization of the sample occurs by collision with free electrons in the plasma. After the sample is ionized, it is accelerated by a large potential (up to $8-10 \mathrm{kV}$ ) and introduced into the mass spectrometer through an interface consisting of sampler and skimmer cones, followed by electrostatic lenses that perform an initial focus of the ion beam. In the interface region, the pressure drops from atmospheric at the plasma torch to approximately $10^{-8} \mathrm{mbar}$ at the entrance slit. Analyzer pressure is $10^{-9}$ mbar. These pressures are maintained with a series of rotary pumps, turbo pumps and ion pumps.

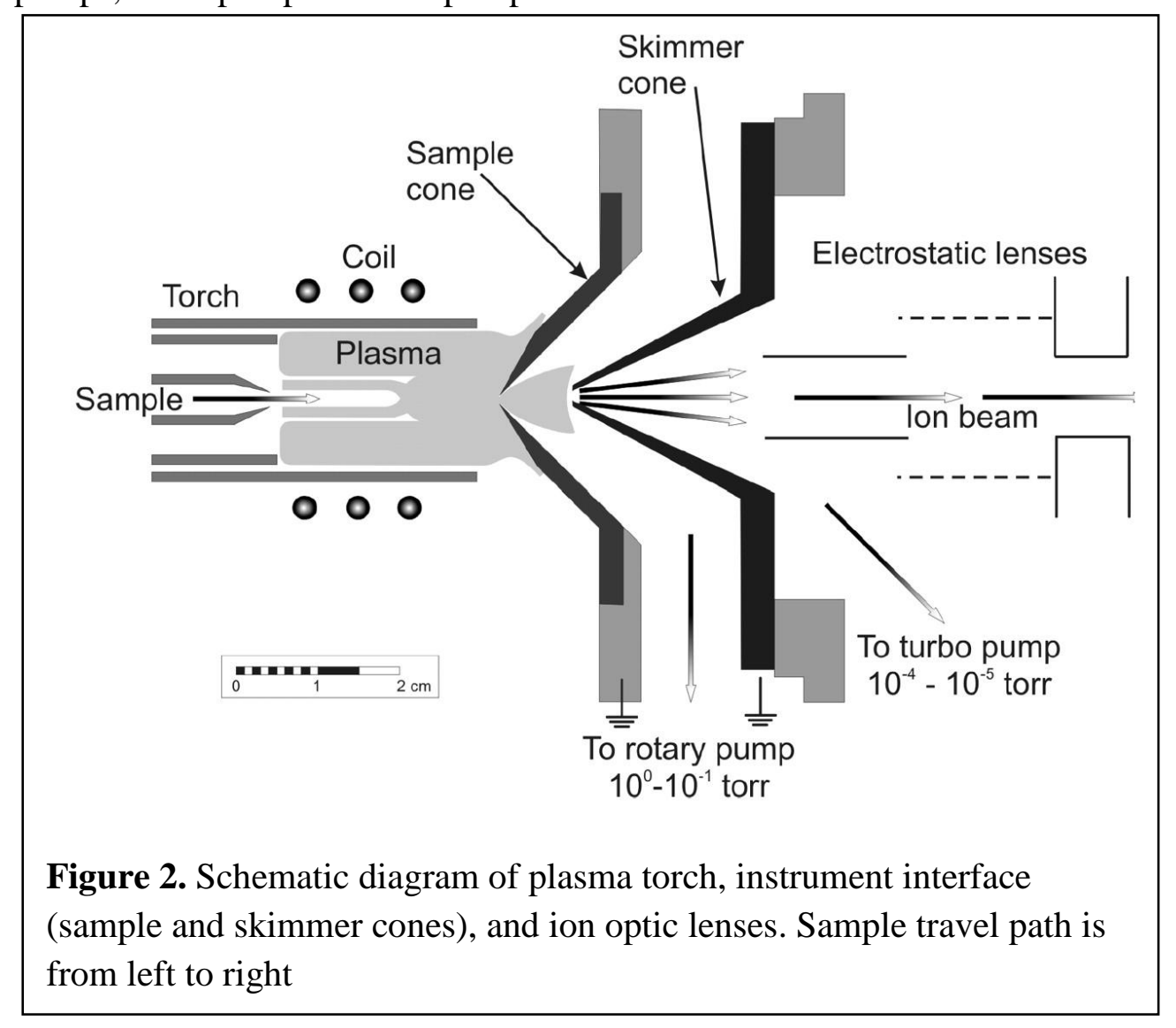

After passing
through interface, the ion beam is focused and passed through an electromagnet (also called a magnetic sector or mass analyzer) to separate the masses for analysis. Although the engineering principles used in focusing an ion beam are the same in all MCICP-MS instruments, the exact configuration of focusing elements is typically proprietary and unique to the particular instrument model and manufacturer. The focusing mechanisms on an MC-ICP-MS work together to reduce both the energy spread and the angular spread of the ion beam. The ion optics provide initial angular focus to the ion beam, and an electrostatic analyzer reduces the energy spread of the ion beam. Mass dispersion is created as the beam passes through a magnetic sector. Final focusing of the ion beams for perfect alignment with the detectors is accomplished with another set of ion lenses. 


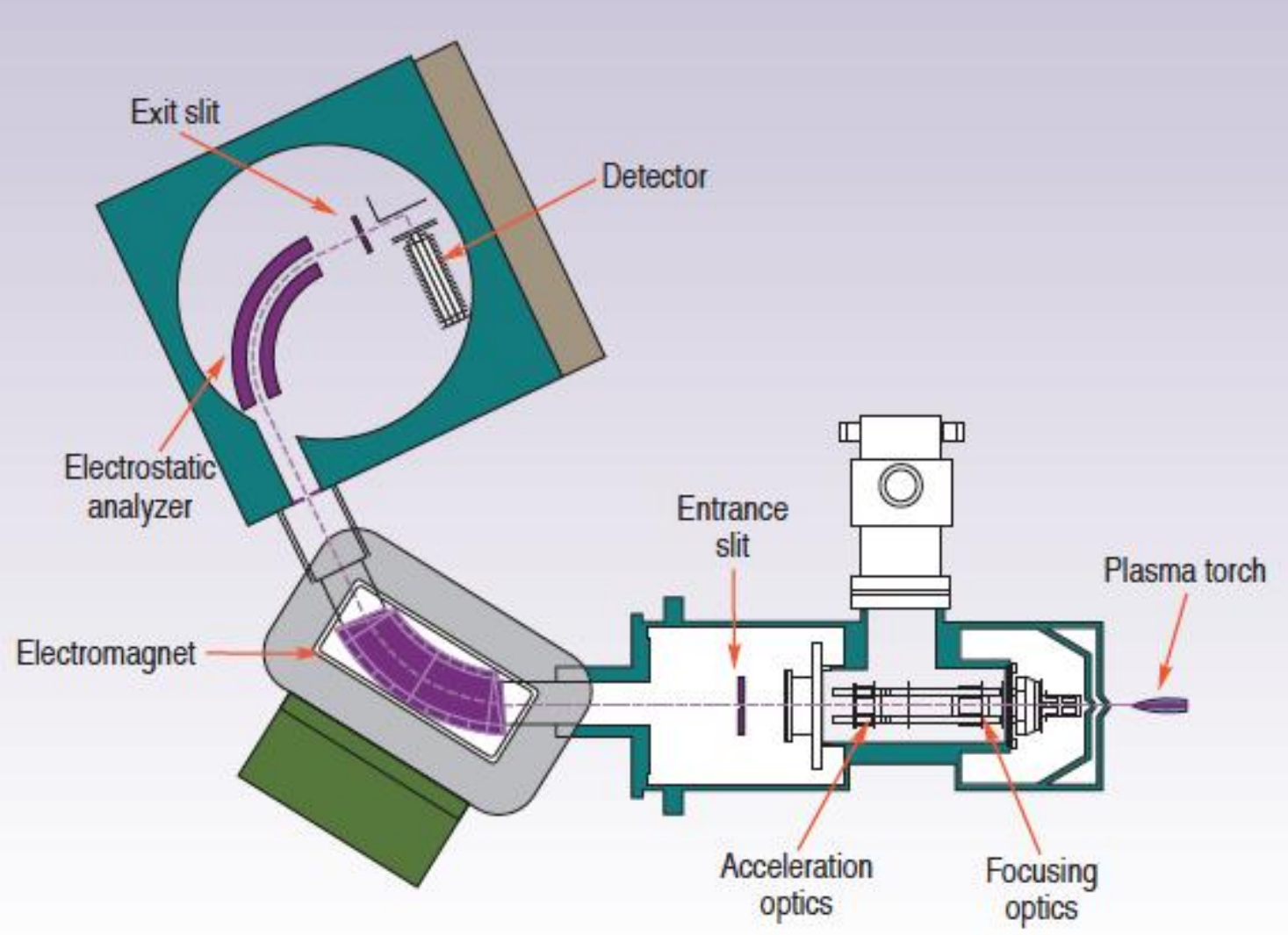

Figure 3. Generalized plan view of a MC-ICP-MS

The ion beam intensities are measured using a collector array of Faraday cups and/or ion counters. These collectors measure the ion beams as voltages, and isotope ratios are calculated from the ratios of the ion beam intensities measured on different detectors. Current state-of-theart mass spectrometers have as many as 16 Faraday collectors and 5 ion counters. Faraday collectors are used to measure ion beams with high intensities, whereas ion counters are used to measure low intensity ion beams. Thus, with a combination of Faraday cups and ion counters, it is possible to measure minor isotopes that are present at the ppm level, relative to the most abundant isotope. For analytes that are commonly present in low abundance in a sample, such as $\mathrm{Pu}$ or other trace actinides, an instrument can be configured with multiple ion counters to measure all masses simultaneously.

\section{Analytical Issues}

Sample preparation. Samples must be chemically purified prior to analysis to isolate the element of interest from the rest of the bulk sample. There are two reasons for this. First, this removes any potential isobaric interferences from the analyte. Potential isobaric interferences 
include atomic as well as molecular species (typically hydride, oxide, nitride or argide). Second, purification removes sample matrix that may affect the ionization efficiency of the sample or the way in which the sample fractionates in the plasma. Because analytical results are commonly corrected for instrumental biases using analyses of standard materials, it is important that the sample matrix is as similar to the standard matrix as possible. The sample amount needed for an analysis depends on the instrument sensitivity, which in turn depends on factors including the sample introduction system, plasma source configuration and the tuning of the ion optics. In general, a single analysis can consume $100 \mathrm{fg}$ to $100 \mathrm{ng}$ of sample, depending on the desired level of precision.

Instrumental mass bias. Ionization of a sample in a plasma and subsequent introduction of the sample into the mass spectrometer generates a mass dependent bias, or mass fractionation, whereby one mass is preferentially sampled over another mass. The causes of this mass bias in MC-ICP-MS instruments are poorly understood and as a result equations describing this mass bias may be imperfect. For most elements in most samples, there are two methods by which mass biases are corrected. In the first method, a ratio of two stable isotopes of an element are assumed to have a known and constant value. Using this 'true' ratio, the other measured mass ratios of the sample are corrected using a mass fractionation law, typically an exponential law. In an alternative method, a correction factor that is calculated from measured standards is applied to samples measured in the same analytical session.

\section{Strengths}

The major strength of MC-ICP-MS is that ionization efficiency of all elements in the plasma is very high, enabling analysis of elements that have very low ionization potentials that would otherwise be extremely difficult to analyze by TIMS. Additionally, although mass fractionation on an MC-ICP-MS is greater than on a TIMS, the plasma operates in a steady state, so that the mass fractionation remains constant through an analytical session. Sample throughput on an MCICP-MS is greater than on a TIMS, allowing analysis of 20-30 samples in a 12-hour analytical session. Finally, the plasma source configuration allows flexibility in sample introduction methods. In addition to solution-based sample introduction systems as described above, a laserablation sample introduction system may also be used, which enables spatially resolved analysis of solid samples.

\section{Weaknesses}

Although multi-collection of ion beams compensates for much of the instability of the plasma source, this instability ultimately limits the precision that may be obtained using MC-ICP-MS. Also, the factors controlling mass fractionation in a plasma are not fully understood. As a result, 
mass fractionation laws used to correct results do not perfectly describe observed mass fractionation. 


\section{High Resolution Gamma Spectrometry}

\section{Introduction}

Gamma spectrometry is a non-destructive technique used to identify and quantify gamma-emitting radionuclides present in samples. Gamma spectrometry takes advantage of the characteristic gamma-ray energies emitted by each radionuclide and the proportionality between

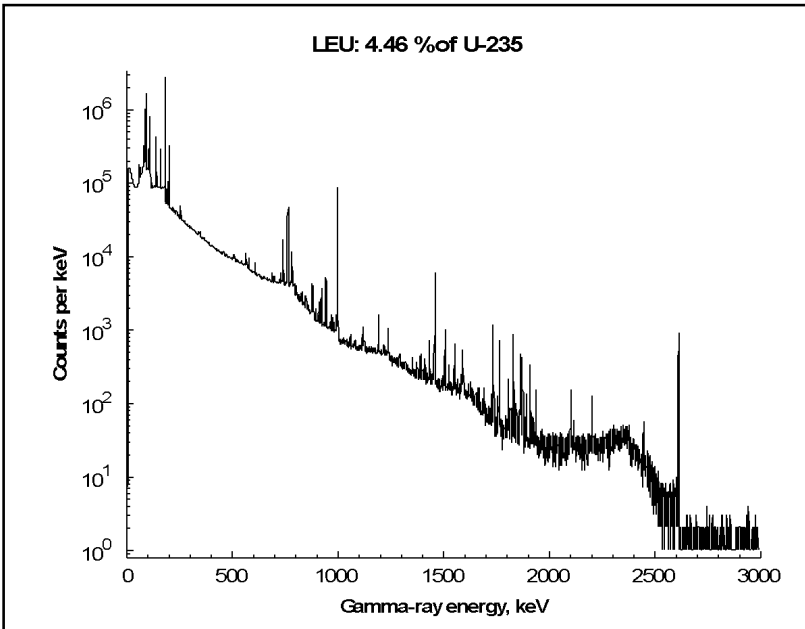

Figure 1. Gamma spectrum of uranium isotopically enriched to $4.46 \%{ }^{235} \mathrm{U}$. (spectra available from the Nucleonica web portal) the intensity of the emitted radiation and the activity of the radionuclide in the sample being measured. A gamma spectrum displays the number of detected gamma photons as a function of energy. Figures 1 and 2 show typical gamma spectra of uranium and plutonium.

\section{Laboratory applications of gamma} spectrometry for nuclear security and nuclear forensics purposes provide a means to accurately determine the isotopic composition of gamma-emitting nuclides in a sample and to quantify the amount of each radioisotope present. In the analysis of special nuclear material, such as uranium and plutonium, gamma spectrometry may also be used to determine the total nuclear material content, the age of the material, the presence of reprocessed uranium, and the presence of fission products. This information can assist an investigation by providing indicators of the origin of the illicit material.

\section{Instrumentation}

A typical state-of-the-art highresolution gamma spectrometry system (Figure 2) consists of a detector crystal made of high-purity germanium (HPGe), cooled either by liquid nitrogen or by an electrical cooler, with a preamplifier,

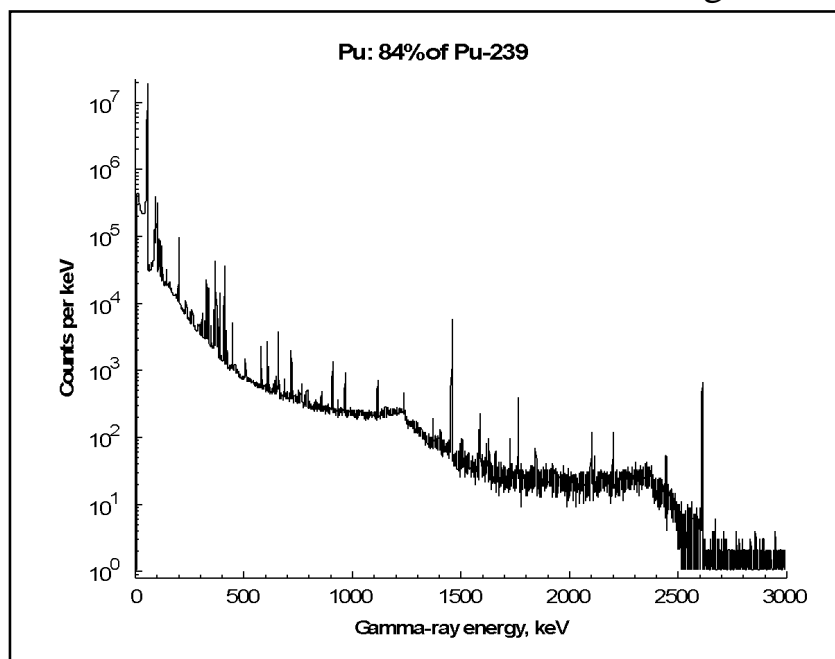

Figure 2. Gamma spectrum of plutonium with 84 $\%{ }^{239} \mathrm{Pu}$ cooling system, electronics unit(s), and a computer for displaying, saving, and analyzing gamma spectra. The electronics units are often integrated into one compact device. In fact, portable systems often have all components integrated into a single hand-held instrument. For specific applications in the laboratory, low- 
background shielding for the detectors is often used in order to isolate the detector from natural background radiation and interferences from other radioactive materials.

In order to collect a gamma spectrum, one places the item under investigation in front of the detector in a configuration specific to the detector orientation and certain sample parameters. Gamma radiation emitted from the sample enters the detector and produces electronic signals

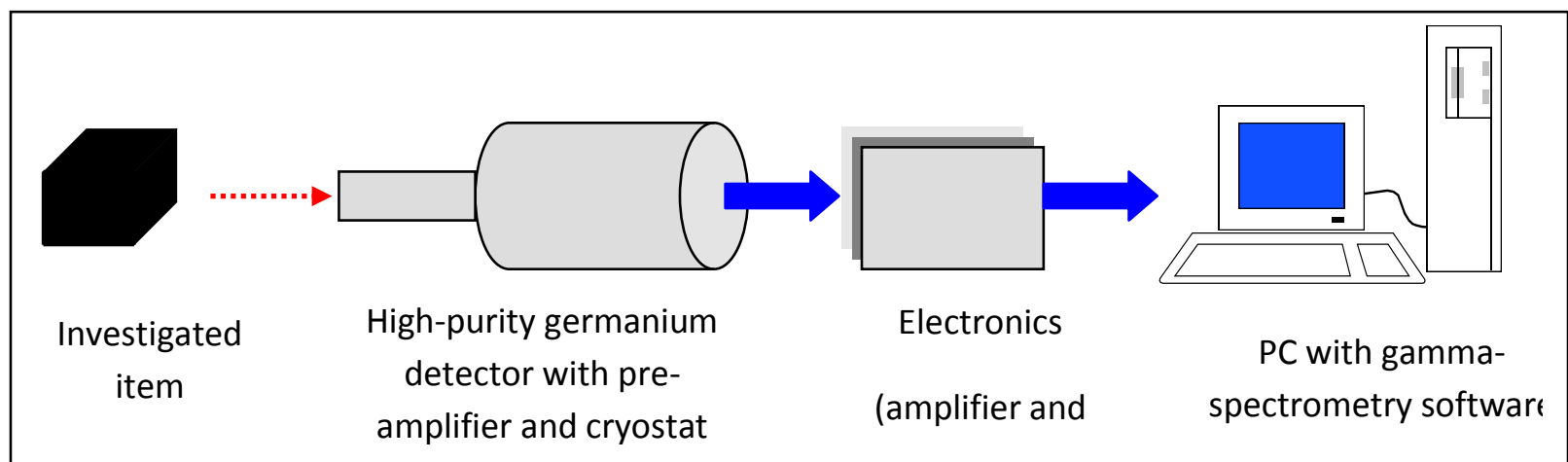

Figure 2. Components of a typical gamma-spectrometry setup.

that are automatically counted and sorted into discrete channels according to their energy by the electronics and software. These data are typically displayed as a histogram of the number of detected signals (photons) in each channel. Converting the $\mathrm{x}$ scale (abscissa) of this histogram from channel number to photon energy yields the acquired gamma spectrum (as displayed in Figures 1a and 2).

\section{Analytical Issues}

The time needed to accumulate a reliable gamma spectrum is variable and depends on properties of the investigated item (e.g., its level of radioactivity, its density), the specifications of the detector (e.g., its efficiency), the measurement configuration, and the information needed to be extracted from the gamma spectrum (nuclide identification versus quantification). For most nuclear forensic scenarios and sample types, the counting time required for on-site categorization is on the order of tens of minutes, while the time required to collect spectra for a detailed nuclear forensic

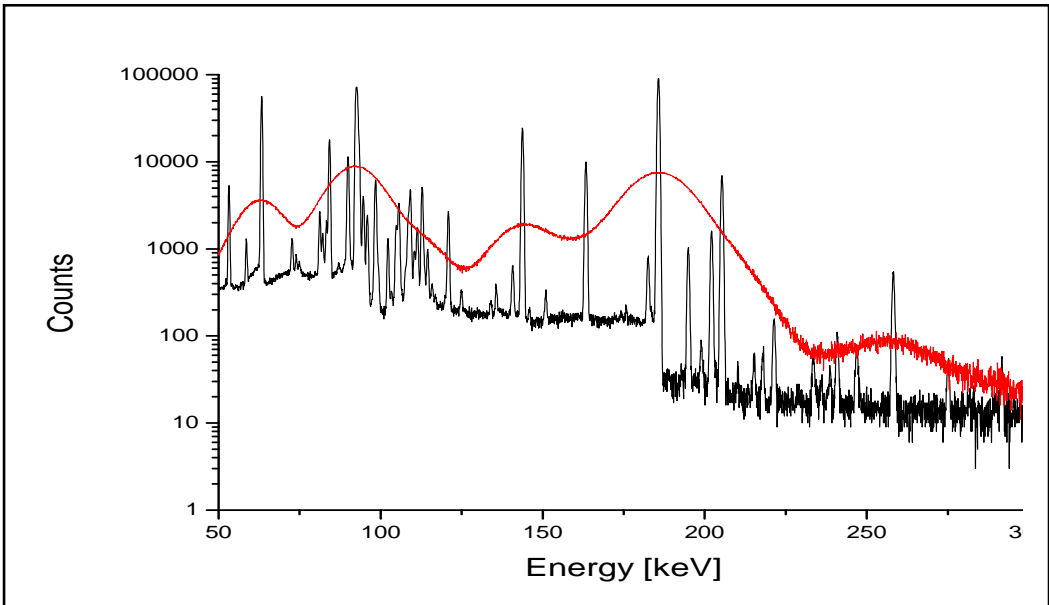

Figure 3. Spectra of low-enriched uranium taken with a lowresolution 
analysis in the laboratory is on the order of hours to days.

An important figure-of-merit for gamma spectrometers is their efficiency. The efficiency of a gamma spectrometer is the ratio of the number detected gamma photons to the number of photons incident on the detector. The higher the efficiency, the shorter the time required to collect a representative spectrum of the investigated sample.

Another important figure-of-merit is the energy resolution of the spectrometer. The energy resolution measures the ability of a spectrometer to distinguish between photons of slightly different energy. It is usually specified as the "Full Width at Half Maximum" (FWHM) of a gamma peak at a predefined energy, for example the $1332 \mathrm{keV}$ peak of ${ }^{60} \mathrm{Co}$. The smaller the FWHM, the higher the resolution and the better adjacent peaks can be resolved in the spectrum. The difference between low-resolution and high-resolution gamma spectra is illustrated in Figure 3. This Guideline is focused on high-resolution gamma spectrometry, because high-resolution is required in order to investigate the parameters relevant to nuclear forensics (see Table 1, below). It should be mentioned, however, that low-resolution gamma spectrometers can also be used, to a limited extent, to identify radioactive sources and to detect the presence of nuclear material.

Unfortunately, the resolution of a gamma spectrometer decreases with increasing efficiency. Therefore, the choice of the appropriate gamma spectrometer is always a compromise between efficiency and resolution.

\section{Application}

Low-resolution and medium resolution detectors, based on, e.g., $\mathrm{Cd}(\mathrm{Zn}) \mathrm{Te}$ semiconductors or on scintillator crystals, such as $\mathrm{NaI}$ or $\mathrm{LaBr}_{3}$, although satisfactory for some in-field applications, cannot provide the accuracy and precision needed for detailed laboratory investigations. High-resolution gamma spectrometers, with detectors made of high-purity germanium (HPGe), are required for laboratory use. Note, however, that even the performance of currently available, electrically cooled, compact portable detector systems is not satisfactory for detailed analyses in the laboratory. For laboratory investigations, only "classical" liquid-nitrogen cooled HPGe detectors may be used.

For each item which requires nuclear forensic characterization, two different types of gamma spectrometric measurements, using two different HPGe detectors, are performed in the laboratory. First, the isotopic composition of $\mathrm{U}$ and/or $\mathrm{Pu}$, as well as the age of $\mathrm{Pu}$, is determined by a gamma spectrometer having good energy resolution for the low energy range around 100 $\mathrm{keV}$ (usually "planar detectors" with resolution (FWHM) $<600 \mathrm{eV}$ at $122 \mathrm{keV}$ ). Additional information about the investigated material can be obtained from the higher energy range. Unfortunately, detectors with good energy resolution in the low-energy range have poor 
efficiency in the higher energy range, so high-energy spectra taken with these detectors are not suitable for analysis. Therefore, another measurement using a high-efficiency (coaxial) detector is also performed in order to obtain information about the age of the $\mathrm{U}$, the presence of reprocessed $U$, and the presence of fission products. In addition, the high-energy spectrum can also confirm the $\mathrm{Pu}$ isotopic composition obtained from the low-energy spectrum.

Note that there are also commercially available "broad energy detectors" which offer relatively good resolution, combined with a relatively high efficiency, that can be used for both purposes, although with some trade-offs in performance. Such broad energy detectors can be a cost-effective solution, especially if the same detector is to be carried into the field. However, for best performance in the laboratory, two separate detectors are required, both optimized for specific purposes.

Because the effects to be measured with the high-efficiency detector in the high-energy range are usually quite small, this detector must be shielded from natural background radiation, which might interfere with the evaluation of the data from the sample itself. For isotopic composition measurements with the low-energy detector, the natural background radiation is negligible, so there is usually no need for low-background shielding. However, in active laboratories, where many other nuclear samples are also handled, a shield for the low-energy detector may also be desirable.

\section{Sample requirements}

Gamma spectrometry is a non-destructive method. Thus, the technique can be applied to a wide range of sample types and configurations. Investigated items can usually be analyzed as they are received without any sample preparation. Isotope-ratio and age determination measurements do not depend on the sample shape, physical form and chemical composition, nor do they require the use of reference samples. When quantification of radioactive material content is the goal of the analysis, measurement configuration becomes important and comparison to a reference sample similar to the investigated item or the use of modeling techniques is required.

For gamma spectrometric measurements, it is usually assumed that the samples are homogenous. However, one must keep in mind that, for very large items, the detected gamma radiation mainly derives from the outer regions of the item. Therefore, only limited information can be obtained about the interior of a large item. Critical sample properties which might influence a gamma spectrometric measurement include: activity of sample, sample shielding, and the presence of other radioactive materials which emit gamma radiation might interfere with the radiation from the investigated material. 


\section{Strengths and weakness}

Strengths. Gamma spectrometry is a fully non-destructive method that preserves other types of forensic evidence. Samples and items can be analyzed without dismantling the item. There is little or no sample preparation required prior to analysis. Gamma spectrometry is relatively inexpensive compared to destructive techniques. The operating costs are also low.

Weakness. The precision is often less than the precision of destructive techniques (the standard deviation for a given measurement result using high-resolution gamma spectrometry will typically be higher). For high-resolution gamma spectrometers, liquid nitrogen cooling is needed. Present-day commercial gamma spectrometers are not able to directly derive information on ${ }^{242} \mathrm{Pu}$ from the measured spectrum. Isotopic correlations have to be used to estimate the ${ }^{242} \mathrm{Pu}$ content of the investigated items. For Pu with high ${ }^{242} \mathrm{Pu}$ content, this causes a large uncertainty in determining the Pu isotopic composition. Note, however, that by combining gamma spectrometry with two other non-destructive methods, calorimetry and neutron coincidence counting, it is possible to derive the ${ }^{242} \mathrm{Pu}$ content in a fully non-destructive way. 


\section{Alpha Spectrometry}

\section{Introduction}

Prior to analysis by alpha spectrometry, the element of interest is chemically separated from the sample matrix (pure uranium or plutonium samples may not require any additional purification prior to mounting) and then deposited on a metal plate (typically Pt or stainless steel) by stippling or electro-deposition [1], or co-precipitated as a fluoride and mounted on a flat disk [2]. The mounted sample is placed in a chamber under vacuum with a detector at a fixed distance from the sample source. As the sample undergoes alpha decay a portion of the alpha particles strike the detector and the events are recorded. The energy range of measurement is set by upper and lower level discriminators. The lower discriminator is often set at $3 \mathrm{MeV}$, since there are no alpha decays of interest below that energy. The upper discriminator is often set at 8 or $10 \mathrm{MeV}$, so as to capture high-energy decays of interest. The efficiency of detection does not depend on the energy of the particle thus the total counts under a peak are related to the isotope ratio in the sample and the branching ratio of the isotope of interest. Peak resolution is on the order of $50 \mathrm{keV}$ or better as determined by the full peak width at half the maximum peak height (FWHM). Alpha spectra of purified uranium spiked with a ${ }^{232} \mathrm{U}$ tracer is shown in the Figure 1 below (on the left) and of plutonium (no tracer added) on the right below (2).

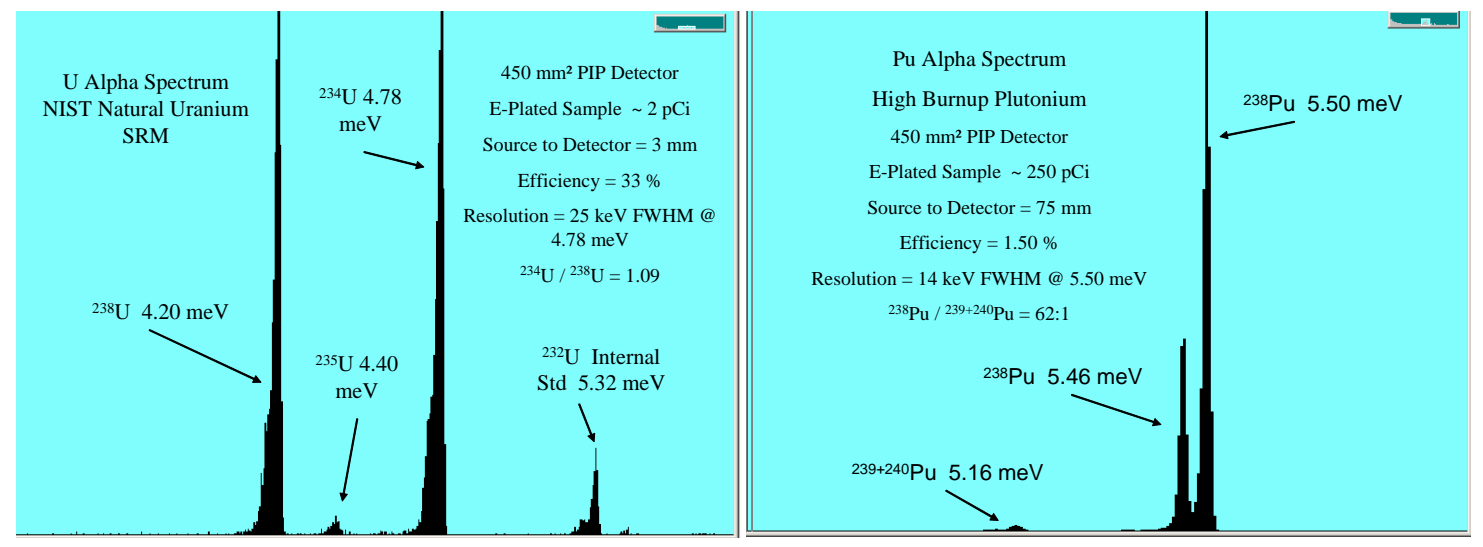

Figure 1 (left) and 2 (right) uranium alpha spectra and plutonium alpha spectra

The instrumentation used to determine and quantify the alpha-emitting nuclide in question is usually a surface-barrier type detector (for low-level nuclear forensic applications) where the energy of the charged particle is deposited in the active volume of the detector. The energy deposited is proportional to the kinetic energy of the alpha particle emitted, which is well known for each isotope, allowing identification and quantification of the isotopes present. Because some alpha decay energies overlap (see Table 1 from [3]), elements are usually chemically separated prior to measurement. A notable exception is that $\mathrm{Am}$ and $\mathrm{Cm}$ isotopes are often measured in 
the same sample aliquot as they are not easily separated by chemical processing and have sufficient differences in their peak energies to be resolved in the spectra. Other impurities should be removed to obtain acceptable resolution of the alpha peaks. Other detector types include a Frisch grid detection system [4] or a liquid scintillation counter [5,6] optimized for alpha measurements; these other systems may be used as screening tools but typically do not provide the lower detection limits and resolution required for nuclear forensics.

Table 1. Alpha particle energies that may be useful in nuclear forensics

\begin{tabular}{|c|c|c|c|c|c|c|c|}
\hline isotope & half-life & energy (keV) & intensity (\%) & isotope & half-life & energy (keV) & intensity (\%) \\
\hline \multirow[t]{2}{*}{ Po-209 } & $102 y$ & 4883 & 80 & Pu-242 & 3.7 e5 y & 4856 & 22.4 \\
\hline & & 4885 & 20 & & & 4900 & 77.5 \\
\hline Po-208 & $290 y$ & 5115 & 100 & Pu-239 & $2.4 \mathrm{e} 4 \mathrm{y}$ & 5105 & 11.6 \\
\hline \multirow[t]{2}{*}{ Po-210 } & $138 d$ & 5304 & 100 & & & 5144 & 15.2 \\
\hline & & & & & & 5157 & 73.4 \\
\hline \multirow[t]{2}{*}{ Th-232 } & $1.4 \mathrm{e} 10 \mathrm{y}$ & 3947 & 21.7 & Pu-240 & $6563 y$ & 5124 & 27.1 \\
\hline & & 4012 & 78.2 & & & 5168 & 72.8 \\
\hline \multirow[t]{2}{*}{ Th-230 } & $7.5 e 4$ y & 4621 & 23.4 & Pu-238 & 87.7 y & 5456 & 29.0 \\
\hline & & 4687 & 76.3 & & & 5499 & 70.9 \\
\hline \multirow[t]{2}{*}{ Th-228 } & $1.91 \mathrm{y}$ & 5340 & 27.2 & Pu-236 & $2.86 y$ & 5721 & 30.6 \\
\hline & & 5423 & 72.2 & & & 5768 & 69.3 \\
\hline \multirow[t]{2}{*}{ U-238 } & $4.5 e 9 y$ & 4156 & 20.9 & Am-242m & $141 y$ & 5142 & 5.8 \\
\hline & & 4198 & 79.0 & & & 5207 & 89.8 \\
\hline \multirow[t]{4}{*}{ U-235 } & $7.0 \mathrm{e} 8 \mathrm{y}$ & 4215 & 5.8 & Am-243 & 7370 y & 5233 & 11.0 \\
\hline & & 4366 & 17.2 & & & 5274 & 87.4 \\
\hline & & 4398 & 55.3 & Am-241 & 432 y & 5443 & 13.1 \\
\hline & & 4596 & 5.1 & & & 5486 & 84.5 \\
\hline \multirow[t]{2}{*}{ U-236 } & $2.3 e 7 y$ & 4446 & 25.9 & & & & \\
\hline & & 4494 & 73.8 & $\mathrm{Cm}-243$ & $29.1 \mathrm{y}$ & 5742 & 11.6 \\
\hline \multirow[t]{2}{*}{ U-234 } & $2.5 e 5 y$ & 4722 & 28.4 & & & 5785 & 72.9 \\
\hline & & 4775 & 71.4 & & & 5992 & 5.7 \\
\hline \multirow[t]{2}{*}{ U-233 } & $1.6 \mathrm{e} 5 \mathrm{y}$ & 4784 & 13.2 & & & 6058 & 4.7 \\
\hline & & 4824 & 84.4 & $\mathrm{Cm}-244$ & $18.1 \mathrm{y}$ & 5762 & 23.6 \\
\hline \multirow[t]{3}{*}{ U-232 } & $68.9 y$ & 5263 & 31.6 & & & 5805 & 76.4 \\
\hline & & 5320 & 68.2 & Cm-242 & $163 d$ & 6069 & 25.1 \\
\hline & & & & & & 6113 & 74.1 \\
\hline \multirow[t]{4}{*}{ Np-237 } & $2.1 \mathrm{e} 6 \mathrm{y}$ & 4639 & 6.2 & & & & \\
\hline & & 4766 & 8.3 & & & & \\
\hline & & 4771 & 25.6 & & & & \\
\hline & & 4788 & 47.9 & & & & \\
\hline \multirow[t]{4}{*}{ Np-235 } & $396 \mathrm{~d}$ & 4925 & 11.6 & & & & \\
\hline & & 4997 & $\sim 6$ & & & & \\
\hline & & 5007 & 24.8 & & & & \\
\hline & & 5025 & 53.8 & & & & \\
\hline
\end{tabular}

\section{Use in Nuclear Forensics}

Alpha spectrometry can be considered complementary to mass spectrometry, but the instrumentation required is much less expensive than a mass spectrometer [7]. Isotopic ratios of uranium can be determined which provide important signatures as to the intended use of the material [8]. Quantification of ${ }^{238} \mathrm{Pu}$ is better accomplished by alpha spectrometry than mass 
spectrometry due to its short half-life and potential mass interference from ${ }^{238} \mathrm{U} ;{ }^{238} \mathrm{Pu}$ may also provide information as to the history and possible use of a material [8]. Alpha spectrometry is not able to separate the peaks due to ${ }^{239} \mathrm{Pu}$ from ${ }^{240} \mathrm{Pu}$ (see Table 1); this is best accomplished by mass spectrometry analysis.

Alpha spectrometry may be used to quantify ${ }^{241} \mathrm{Am}$ (daughter of ${ }^{241} \mathrm{Pu}$ ), which can then be used to calculate the date of the last plutonium purification performed on a sample (assuming complete purification). Thorium-230 (daughter of ${ }^{234} \mathrm{U}$ ) can similarly be used to determine a last purification date for uranium materials. The presence of ${ }^{237} \mathrm{~Np}$ and higher actinides may be determined by alpha spectrometry and are useful to understand the reactor in which the material was produced.

Polonium-210 is naturally occurring; it may also be made in a nuclear reactor for military or commercial purposes. Recently, it was the "poison" used to kill Alexander Litvinenko in the UK in 2006 [9]. Polonium-210 is an alpha emitter; hundreds of alpha spectrometry measurements were made in the investigation following Litvinenko's death.

\section{Sample Requirements}

In order to quantify the desired radioisotopes in a sample, the element of interest should be chemically separated from other elements so that no spectral interferences occur (e.g. ${ }^{234} U$ can overlap ${ }^{237} \mathrm{~Np}$ and ${ }^{241} \mathrm{Am}$ can overlap ${ }^{238} \mathrm{Pu}$ ) and so that the deleterious effect on peak width and resolution due to remaining matrix elements is minimised. Chemical separation and purification methods are often the same as used to prepare samples for thermal ionization mass spectrometry. Following purification, the sample may be electro-deposited on the disk in a diluted salt matrix, usually ammonium sulfate. [1] In this case, following alpha spectrometry the sample may be stripped from the disk and, with further purification, analyzed by mass spectrometry. Alternatively, the purified sample can be precipitated as a rare earth fluoride and collected on a filter for counting. [2] A maximum sample loading of $\sim 1$ microgram of total uranium should be followed to obtain a thin deposit and ensure spectral resolution is not deteriorated by the thickness of the sample deposit. If detectors are kept free of contamination and counting times of several days are used, detection limits on the order of fCi $(10$ e-15 $\mathrm{Ci} ; \mu \mathrm{Bq})$ are attainable. More typical counting times of 1000 minutes result in detection limits of $0.1 \mathrm{pCi}(3.7 \mathrm{mBq})$ or lower.

\section{Strengths and weaknesses}

Alpha spectrometry is the preferred technique for the alpha emitting radioisotopes with halflives less than a few 100 years, e.g., ${ }^{210} \mathrm{Po},{ }^{232} \mathrm{U},{ }^{238} \mathrm{Pu}, \mathrm{Am}$, and $\mathrm{Cm}$ isotopes. It can be used to determine the very long-lived actinides if a mass spectrometer is not available. It is complimentary to TIMS and MCICPMS measurement for isotope ratio determinations. Precision of less than a few percent is obtainable using clean detectors (detectors free from all 
contamination; detector contamination is often due to recoil from previously counted high activity samples or quality control checks) and counting times of a day (1000 minutes).

+ Inexpensive instrumentation

+ Small footprint for detectors and associated electronics (can be physically stacked and multiple detectors controlled by a single computer)

+ Good detection limits (sub-pCi, $\mathrm{mBq}$ ) for shorter-lived alpha emitting isotopes ( $<500$ years)

+ Can be used for any alpha emitting radionuclide

- Sample preparation is rather time consuming and is similar to mass spectrometry

- Long counting times (1 to several days)

- Spectral overlap does not typically allow separation of ${ }^{239} \mathrm{Pu}$ and ${ }^{240} \mathrm{Pu}$, nor ${ }^{235} \mathrm{U}$ from ${ }^{236} \mathrm{U}$ (high activity samples counted using a sample to detector distance of $>3 \mathrm{~cm}$ may allow some spectral deconvolution) 


\section{Isotope Ratio Mass Spectrometry}

\section{Introduction}

Isotope ratio mass spectrometry (IRMS) is a destructive technique used to measure small variations in the isotopic compositions of light elements. IRMS is a highly specialized technique, which focuses on the isotopic composition of just a handful of elements, but does so with great precision and accuracy. Common elements analyzed by IRMS and their isotopic abundances are listed in Table 1. For most elements the isotopic distribution consists of a major isotope followed by one or more minor isotopes, and IRMS instruments are typically configured with these abundance differences in mind.

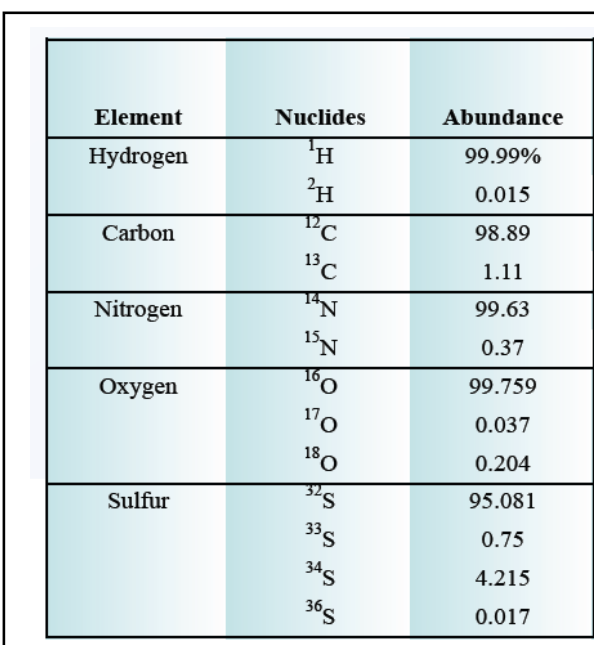

Table 1. Isotopic abundances of elements commonly measured by IRMS.

\section{Instrumentation}

IRMS has been around since the late 1940's. Since it's invention, IRMS instruments have been refined to make them more stable, reliable and somewhat more user friendly. IRMS instruments typically operate at high vacuum with pressures ranging from $10^{-6}$ to $10^{-8}$ mbar. The instrument consists of a source, a magnetic sector mass analyzer, and a collector assembly. The instrument parameters are configured and optimized based on the element of

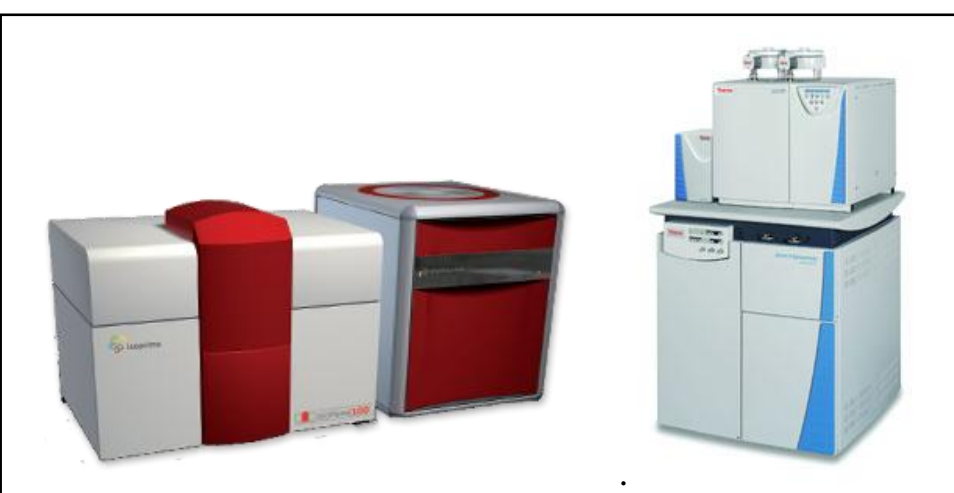

Figure 1. Commercially available IRMS systems interest. For example, different voltages, magnetic current, and collector locations are typically used for hydrogen versus carbon dioxide. Most instruments are only capable of analyzing one gas at a time, but modern instruments can be switched from one gas to another electronically using an automated computer program.

Source. Samples are introduced as a gas into the ionization chamber, which houses the source where the sample gas is ionized, focused and accelerated. The inlet is commonly a capillary that permits viscous flow into the ionization chamber, and acts to reduce the pressure 
from atmospheric down to high vacuum pressures. The sample gas inlet leads into an electron impact source, typically a hot tungsten filament, where positive ions are generated. These ions are then accelerated to about $10 \mathrm{kV}$ using charged plates. Additional lenses and plates are used to focus and steer the ion beam into the flight tube.

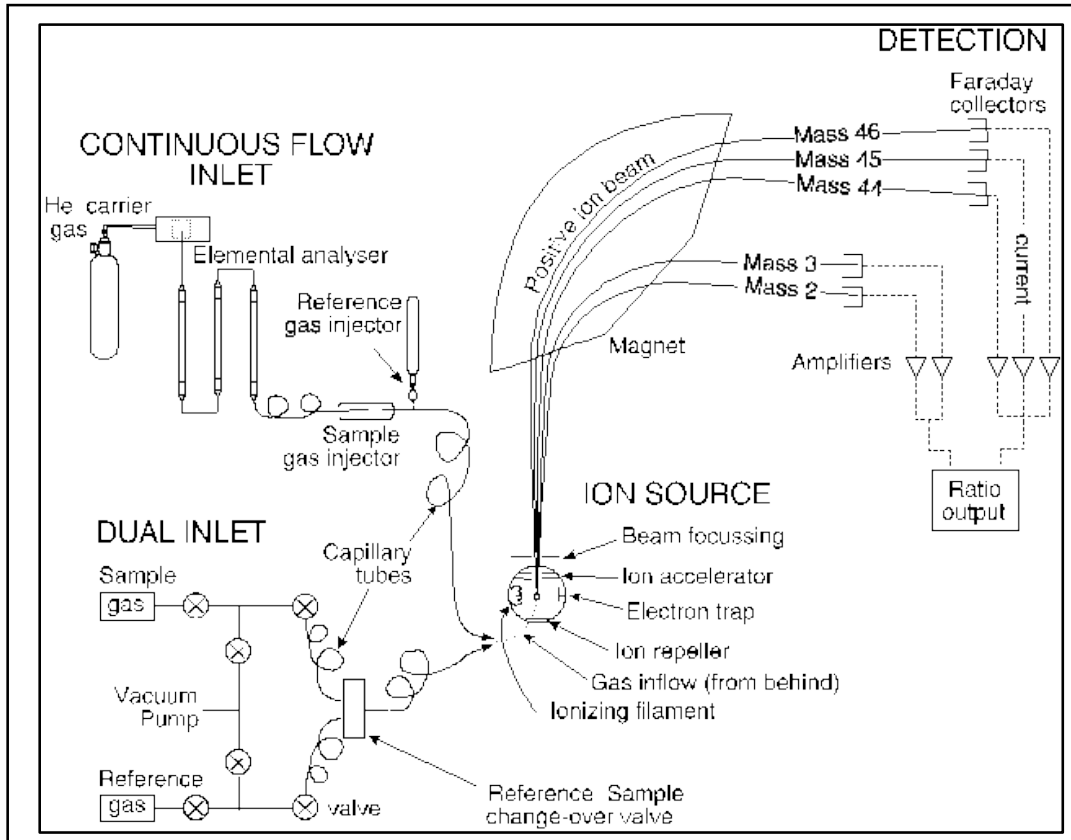

Figure 2. Diagram of IRMS with both dual inlet and continuous flow sample introduction. From Clark and Fritz (1997, Environmental Isotopes in Hydrogeology, CRC press.

\section{Mass analyzer.} Ions are separated by their mass/charge $(\mathrm{m} / \mathrm{z})$ ratio in a magnetic sector mass analyzer, which consists of a fixed magnet or electromagnet placed over a 60-180 degree bend in the flight tube. Instruments with electromagnets can vary the magnetic field in order to select which $\mathrm{m} / \mathrm{z}$ will arrive at the collector assembly. In general, the intensity of the magnet is required to increase as the mass of the gas molecule of interest increases.

Collectors. Faraday cup collectors are located at the end of the flight tube. These collectors register a small current as the ions are neutralized. These currents are then converted to a digital signal that is continuously monitored by a computer. The number of collectors and their positions are determined by the molecular composition and isotope distribution of the sample gas. For example, for carbon dioxide, three collectors are used to measure masses 44, 45, and 46, which predominantly consist of ${ }^{12} \mathrm{C}^{16} \mathrm{O}_{2},{ }^{13} \mathrm{C}^{16} \mathrm{O}_{2}$, and ${ }^{12} \mathrm{C}^{16} \mathrm{O}^{18} \mathrm{O}$. Other minor isotopes may also contribute to the signal, for example ${ }^{12} \mathrm{C}^{16} \mathrm{O}^{17} \mathrm{O}$ also has a mass of 45 . These contributions are commonly corrected for by assuming a natural abundance ratio of ${ }^{18} \mathrm{O}$ to ${ }^{17} \mathrm{O}$. The collector system is further configured to the gases of interest by the resistors selected for the amplifier, which determine the signal resulting from detection of an ion. Lower resistance is used for abundant isotopes such as mass 44 in the example of $\mathrm{CO}_{2}$, and higher resistance is used for the minor species such as masses 45 and 46 from $\mathrm{CO}_{2}$. 


\section{Analytical Issues}

Standardization. IRMS systems are typically optimized to make highly precise and accurate measures of isotope compositions. IRMS systems are usually not designed to measure absolute isotopic abundances. Rather, the isotopic compositions of unknown samples are compared with those of a reference substance. In practice, this is accomplished by measuring the isotope ratios of a reference gas under the same conditions as a sample gas. The reference gas is calibrated against an internationally recognized standard. This can be accomplished by directly analyzing the reference gas against a gas with known isotopic composition, or by running primary or secondary standards through the sample preparation process.

Since isotope ratios are measured relative to a standard, the result of an isotopic analysis is given as a delta value ( $\square$ ), which is the difference between the sample and the reference material. Because the natural isotopic variations are small, delta values are conventionally calculated as per mil (mil meaning 1000), and noted as \%o.

$$
=\frac{R_{\text {sample }}}{R_{\text {std }}} 11000
$$

Where $\mathrm{R}_{\text {sample }}$ and $\mathrm{R}_{\text {std }}$ are the isotope ratios (e.g., ${ }^{2} \mathrm{H} /{ }^{1} \mathrm{H},{ }^{13} \mathrm{C} /{ }^{12} \mathrm{C},{ }^{18} \mathrm{O} /{ }^{16} \mathrm{O}$, etc.) of the sample and reference standard.

The international standards for IRMS analysis are listed in Table 2. Primary and secondary reference materials that are directly tied to these substances are available from the IAEA, NIST and others. However, not all materials have a reference material available that matches the form of the chemical form of the sample. For example, there is currently no internationally recognized stable isotope standard for oxygen in uranium oxide. Furthermore, the international standard for oxygen is VSMOW, or Vienna Standard Mean Ocean Water, a liquid. In this example, oxygen isotope analyses of uranium oxide samples must be calibrated against the available standard reference materials that most closely match. In this case, the closest match is NBS-28, a quartz sand. Laboratory reference materials, known as a working standards, can then be calibrated against the available reference material.

Table 2. IRMS standards.

\begin{tabular}{|l|l|}
\hline Element & Standard \\
\hline Hydrogen & Vienna Standard Mean Ocean Water (VSMOW) \\
\hline Carbon & Vienna PeeDee Belemnite (VPDB) \\
\hline Nitrogen & Atmospheric nitrogen (AIR) \\
\hline Oxygen & Vienna Standard Mean Ocean Water (VSMOW) \\
\hline Sulfur & Canyon Diablo Troilite (CDT) \\
\hline
\end{tabular}


Gas purity. IRMS systems rely on chemically pure reference and sample gases for accurate analyses. The presence of impurities can lead to interferences that degrade measurement quality. For dual inlet systems, this means that offline preparation techniques must be thorough, and involve purification steps, usually in the form of cryogenic separations. For continuous flow systems, peripheral instruments must employ gas chromatography or purge trap columns to separate gas mixtures prior to introduction to the IRMS. Online systems also commonly employ moisture and chemical traps to purify the gas stream. Care must be taken to ensure that the purification steps are functioning, and that the sample preparation process does not fractionate the sample gas.

\section{Application}

IRMS systems are designed to analyze gases. This means that samples of interest that are liquids, solids, or mixtures thereof, must first be converted to an appropriate gas by either online or offline techniques. Sample preparation for online techniques often only require aliquoting an appropriate weight of sample material. Offline sample preparation techniques are sometimes quite complex and rely on extensive procedures to convert the sample of interest into a gas and purify it using vacuum line techniques.

There are two configurations of gas inlets for IRMS systems, dual inlet and continuous flow. The first IRMS systems used a dual inlet configuration. The dual inlet IRMS employs two variable volume bellows. One bellows holds a reference gas, and the other bellows holds the sample gas. The bellows are adjusted so that the pressure is equal, and a switching valve is used to alternate between the reference and sample gases to obtain multiple measurements of the isotopic ratios. The dual inlet system allows for very high precision measurements $(<0.1$ per mil), but requires offline preparation of a pure gas from the original sample of interest. Typically, offline preparation requires vacuum line chemistry and purification processes. These offline techniques are often time consuming, and require more sample material than online procedures.

More recently, continuous flow IRMS has been used to lower sample size requirements and increase sample throughput. Continuous flow IRMS uses a continuous gas stream, usually helium, to carry the sample gas into the IRMS inlet. Compared with dual inlet IRMS systems, continuous flow systems are typically lower precision (0.1-0.3 per mil). However, continuous flow IRMS provides a significant advantage by allowing for sample gas preparation to be done online by interfacing with additional peripheral instruments. Gases in a mixture can be separated and purified by gas chromatography columns prior to introduction at the IRMS inlet. Solids and liquids can be combusted or pyrolyzed to make a gas using an automated peripheral instrument, which significantly reduces sample preparation time. This has led to numerous hyphenated instruments by combining IRMS with peripherals such as elemental analyzers (EA-IRMS), gas chromatography followed by combustion (GC-C-IRMS), and liquid chromatography (LCIRMS). 


\section{Sample requirements}

Bulk samples should be homogenous. Heterogeneities in the sample can result in spurious results and/or poor precision. Typically, $0.1-50 \mathrm{mg}$ of sample material is required, depending on the elements of interest and the chemical composition of the material. Samples can be solid, liquid or gas.

\section{Strengths and weaknesses}

Strengths. IRMS systems are capable of achieving high precision and accuracy. The sample size requirements and preparation procedures are well suited for analysis of bulk solids. Automated online IRMS systems require very little sample preparation, and allow for high sample throughput.

Weaknesses. Some analyses such as oxygen in oxides can only by done offline, and are more time consuming and require substantial safety controls. IRMS cannot measure isotopic compositions over the small spatial scales achievable by SIMS for in situ analysis of small particles or small areas within a sample. Recent innovations in cavity ring down spectroscopy systems may allow for more user-friendly measurement of stable isotope compositions in some materials. 


\section{Discussion of Analytical Techniques}

Optical Microscopy - Naomi Marks

X-ray Diffraction - Sarah Roberts

Scanning Electron Microscopy - Jonathan Plaue

Transmission Electron Microscopy - Zurong Dai

Optical Spectroscopy - Greg Klunder

Electron Microprobe - Naomi Marks

Secondary Ion Mass Spectrometry - Mike Kristo/Lars Borg

X-ray Fluorence - Gary Eppich

Davies-Gray Titration - Gary Eppich

Quadrupole Inductively Coupled Mass Spectrometry - Rachel Lindvall

Multi-collector Inductively Coupled Mass Spectrometry - Amy Gaffney

Thermal Ionization Mass Spectrometry - Lars Borg

Isotope Ratio Mass Spectrometry - Mike Singleton

UF 6-Mass Spectrometry - Ross Williams

-Spectroscopy - Mike Kristo/Lars Borg 